\title{
DIFFEOMORPHIC IMAGE REGISTRATION WITH AN OPTIMAL CONTROL RELAXATION AND ITS IMPLEMENTATION*
}

\author{
JIANPING ZHANG ${ }^{\dagger}$ AND YANYAN LI ${ }^{\ddagger}$
}

\begin{abstract}
Image registration has played an important role in image processing problems, especially in medical imaging applications. It is well known that when the deformation is large, many variational models cannot ensure diffeomorphism. In this paper, we propose a new registration model based on an optimal control relaxation constraint for large deformation images, which can theoretically guarantee that the registration mapping is diffeomorphic. We present an analysis of optimal control relaxation for indirectly seeking the diffeomorphic transformation of Jacobian determinant equation and its registration applications, including the construction of diffeomorphic transformation as a special space. We also provide an existence result for the control increment optimization problem in the proposed diffeomorphic image registration model with an optimal control relaxation. Furthermore, a fast iterative scheme based on the augmented Lagrangian multipliers method (ALMM) is analyzed to solve the control increment optimization problem, and a convergence analysis is followed. Finally, a grid unfolding indicator is given, and a robust solving algorithm for using the deformation correction and backtrack strategy is proposed to guarantee that the solution is diffeomorphic. Numerical experiments show that the registration model we proposed can not only get a diffeomorphic mapping when the deformation is large, but also achieves the state-of-the-art performance in quantitative evaluations in comparing with other classical models.
\end{abstract}

Key words. Diffeomorphic image registration; Dynamical system; Jacobian equation; Optimal control relaxation; Augmented Lagrangian multiplier method; Grid unfolding indicator; Deformation correction.

AMS subject classifications. 49K20, 57N25, 65F22, 65N50, 74G65, 94A08, 94A12, 92C50, 92C55

1. Introduction. Image registration refers to the process of finding a spatial transformation between two or more images according to a certain registration measure, so that the corresponding points of two images can be spatially consistent. Image registration is one of the most basic problems in computer vision and also is a difficult problem in medical image analysis. It has been widely used in target tracking, geological exploration, disease detection, angiography and other fields $[10,33,37,40,54]$. Therefore, medical image registration has a vital role in promoting the research of clinical medicine $[5,27,39,45]$.

Non-rigid registration is suitable for the non-rigid object deformation, and can achieve local deformation more accurately. The commonly used models of non-rigid registration include: total variation (TV) model [19, 20], diffusion model [16], curvature model [17], elastic model [6, 18], viscous fluid model [13] and optical flow field model [40] etc. Fischler and Elschlager [18] proposed an embedding elastic metric to constrain relative movement. Bajcsy and Kovacic [2] used affine transformation to correct large global differences before elastic registration. But the elastic model does not allow for large image deformations due to its local nature. Christensen et al. [13, 14] proposed a viscous fluid regularization term to replace the elastic energy. Such model can deal with large deformation, but it can not generate diffeomorphism. In addition, high computational complexity seriously hampers its application. The Demons registration model was first proposed by Thirion [46]. It is a non-parametric non-rigid registration model that is improved based on the optical flow equation, which is more efficient than other non-rigid registration methods. As pointed

\footnotetext{
*Manuscript Version 1, SePtember 15, 2021.

Funding: This work was supported by the National Natural Science Foundation of China (NSFC Project number 11771369), also partly by grants from the outstanding young scholars of Education Bureau of Hunan Province, P. R. China (number 17B257) and Natural Science Foundation of Hunan Province, P. R. China (number 2018JJ2375, 2017SK2014, 2018XK2304).

${ }^{\dagger}$ School of Mathematics and Computational Science, Hunan National Center for Applied Mathematics, and Hunan Key Laboratory for Computation and Simulation in Science and Engineering, Xiangtan University, Xiangtan, Hunan 411105, P. R. China (Corresponding author, jpzhang@xtu.edu.cn).

${ }^{\ddagger}$ School of Mathematics and Computational Science, Xiangtan University, Xiangtan, Hunan 411105, P. R. China (liyanyan@smail.xtu.edu.cn).
} 
out by Fischer and Modersitzki [16], implementation for diffusion registration is based on finite difference approximation of a diffusion-like equation. One of the most competitive features of this method is its fast speed, which makes such scheme very attractive for high-resolution applications. Curvature-based image registration is a fully automatic and non-rigid registration method proposed by Modersitzki [17], which relies on a curvature based regularization term and provides a smooth solution. In contrast to many other nonlinear registration techniques, the curvature model does not require an additional affine linear pre-registration step. Zhang and Chen [55] presented a novel variational framework of the total fractional-order variation to supervise nonlocal deformation in non-rigid image registration. These registration models can be effective for relatively small deformation, but not all models are effective for large deformation, and in particular few models can be guaranteed to generate diffeomorphic mapping.

Vercauteren [48] proposed a non-parametric diffeomorphic demons image registration algorithm. Christensen et al. [14] developed a large deformation model for computing transformations, which overcomes the limitations of the small deformation model and ensures that the transformations are diffeomorphic. Later, large deformation diffeomorphic metric mapping (LDDMM) framework that can generate the diffeomorphic transformation of image registration was proposed $[4,38,47]$. Lam and Lui [31] introduced a novel model involving a Beltrami coefficient term to obtain diffeomorphic image or surface registrations via quasi-conformal maps, which can deal with large deformations. In [32], a notion of conformality distortion of a diffeomorphism in the $n$-dimensional Euclidean space has also been formulated.

In the past few years, more and more scholars have paid attention to diffeomorphic image registration. The folding degree of deformed mesh in diffeomorphic image registration, measured by the local quantity $\operatorname{det}(\nabla \boldsymbol{\varphi}(\boldsymbol{x}))$, should be reduced or avoided, where $\boldsymbol{\varphi}(\boldsymbol{x})$ is the transformation in image registration, and $\operatorname{det}(\nabla \boldsymbol{\varphi}(\boldsymbol{x}))$ is the Jacobian determinant of $\boldsymbol{\varphi}(\boldsymbol{x})$, especially $\operatorname{det}(\nabla \boldsymbol{\varphi}(\boldsymbol{x}))>$ 0 restricts that the transformation $\boldsymbol{\varphi}(\boldsymbol{x})$ is an one-to-one mapping.

Haber and Modersitzki [25] in 2004 proposed a volume-preserving registration model by limiting that $\operatorname{det}(\nabla \boldsymbol{\varphi}(\boldsymbol{x}))$ equals to 1. Although such incompressibility has important applications in some fields, it is not necessary or reasonable in others. Karacal et al. [30] modified the deformation constraint to satisfy $\operatorname{det}(\nabla \boldsymbol{\varphi}(\boldsymbol{x}))>0$, which ensures the topology preservation of the deformation field $\boldsymbol{\varphi}(\boldsymbol{x})$. Yanovsky et al. [51] applied the symmetric Kullback-Leibler distance to quantify $\operatorname{det}(\nabla \boldsymbol{\varphi}(\boldsymbol{x}))$ for seeking a diffeomorphic mapping. Chen and Oktem [11] developed a variational framework for indirect image registration, where a template is registered against a target that is known only through indirect noisy observations. Zhang and Chen [53] proposed a novel diffeomorphic model for image registration that establishes a link between the Beltrami coefficient of the transformation $\boldsymbol{\varphi}(\boldsymbol{x})$ and the quantity $\operatorname{det}(\nabla \boldsymbol{\varphi}(\boldsymbol{x}))$.

Liao [34] in 2008 proposed the optimal control method for data alignment. The div-curl system was used as a constraint term to ensure the diffeomorphism of the deformation field. Hsiao [28] proposed a new registration method based on the div-curl system, and obtained a Laplace equation about deformation field that satisfies the homogeneous Dirichlet boundary condition. A gradient descent method is given to optimize the transformation $\varphi$.

In this paper, we aim to reformulate an optimal control relaxation dealing with the partial differential equation of Jacobian determinant $\operatorname{det}(\nabla \boldsymbol{\varphi}(\boldsymbol{x}))=f(\boldsymbol{\varphi}(\boldsymbol{x}))$ proposed by Dacorogna and Moser [15], to model diffeomorphic image registration problem. Our contributions are summarized as follows.

- We propose a novel optimal control relaxation technique solving the diffeomorphic image registration model (denoted as OCRDIR) for large deformation images, that establishes a link between the registration transformation and the Jacobian equation, which can theoretically guarantee that the registration mapping is smooth, invertible and also diffeomorphic.

- We present an analysis of optimal control relaxation for indirectly seeking the diffeomorphic 
transformation of Jacobian determinant equation. We also provide an existence result for the minimum of the control increment non-convex optimization in our OCRDIR model.

- We propose a fast ALMM iterative scheme to solve the control increment optimization problem. The main novelty is the applicability of a general framework of constraint optimization tools, which we can apply to reformulate the minimization problem. We also analyze the convergence of the proposed scheme.

- We give a grid unfolding indicator for cell central-difference discretization, then a robust ALMM-based OCRDIR algorithm for using the deformation correction and backtrack strategy is proposed to guarantee that the solution is diffeomorphic. Numerical experiments show that the proposed method can not only get a diffeomorphic mapping when the deformation is large, but also achieves the state-of-the-art performance in quantitative evaluations in comparing with other classical models.

The rest of the paper is organized as follows. In Section 2 we review some methods of image registration modeling. In Section 3, we build some optimal control relaxation theories to indirectly seek the diffeomorphic transformation of Jacobian determinant equation. Our new ALMM-based OCRDIR method is proposed and the convergence analysis is followed in Section 4. We show experimental results in Section 5. Finally we conclude this work in Section 6.

2. Reviews. The task of finding a suitable transformation $\varphi: \Omega \subset \mathbb{R}^{d} \rightarrow \mathbb{R}^{d}(d=2$ or 3$)$ such that a transformed version $T(\boldsymbol{\varphi}(\boldsymbol{x}))$ of the template image $T(\boldsymbol{x})$ is similar to the reference image $R(\boldsymbol{x})$, is to solve a general minimization problem

$$
\min _{\varphi} \mathcal{D}[R, T ; \varphi]
$$

where $\Omega$ is a bounded closed set and $\boldsymbol{u}(\boldsymbol{x})=\boldsymbol{\varphi}(\boldsymbol{x})-\boldsymbol{x}$ is the increment field (i.e., $T(\boldsymbol{\varphi}(\boldsymbol{x}))=$ $T(\boldsymbol{x}+\boldsymbol{u}(\boldsymbol{x}))), \mathcal{D}[R, T ; \boldsymbol{\varphi}]$ is the similarity measure. It is well-known that the problem (2.1) is ill-posed [40], therefore, the classical mathematical model of image registration can be given by

$$
\min _{\varphi}\{\mathcal{E}(\varphi):=\mathcal{D}[R, T ; \varphi]+\tau \mathcal{S}[\varphi]\}
$$

where $\mathcal{S}[\varphi]$ is a regularization term, and $\tau$ is a regularization parameter for managing the trade-off between $\mathcal{D}[R, T ; \boldsymbol{\varphi}]$ and $\mathcal{S}[\boldsymbol{\varphi}]$. Throughout the paper, we choose the so called sum of squared differences (SSD) [40] defined by

$$
\mathcal{D}_{\mathrm{SSD}}[R, T ; \boldsymbol{\varphi}]=\frac{1}{2} \int_{\Omega}(T(\boldsymbol{\varphi}(\boldsymbol{x}))-R(\boldsymbol{x}))^{2} d \boldsymbol{x}=\frac{1}{2} \int_{\Omega}(T(\boldsymbol{x}+\boldsymbol{u}(\boldsymbol{x}))-R(\boldsymbol{x}))^{2} d \boldsymbol{x},
$$

as similarity measure between $T$ and $R$. We also refer readers to literatures [26, 43, 42] for many other similarity measures in mono-modality or multi-modality image registration.

2.1. Image registration modeling. As remarked, various ways of modeling non-rigid image registration have been proposed. Below we review a few of them.

Active demons. Demons algorithm is a well-known non-parametric image registration method proposed by Thirion [46], it combines the optical flow image matching idea with the thermodynamic molecular diffusion theory proposed by Maxwell to image registration. For any point $\boldsymbol{x} \in \Omega$ in the reference image $R$, the matching point $\boldsymbol{\varphi}(\boldsymbol{x})=\boldsymbol{x}+\boldsymbol{u}(\boldsymbol{x}) \in \Omega$ is found one-to-one in the template image $T$ such that $\mathcal{D}[R, T ; \boldsymbol{\varphi}(\boldsymbol{x})]=\frac{1}{2} \int_{\Omega}[T(\boldsymbol{x}+\boldsymbol{u}(\boldsymbol{x}))-R(\boldsymbol{x})]^{2} d \boldsymbol{x} \approx 0$, the second-order 
Taylor expansion of $\mathcal{D}[R, T ; \boldsymbol{\varphi}(\boldsymbol{x})]$ at the origin $\boldsymbol{u}(\boldsymbol{x})=\mathbf{0}$ is obtained

$$
\begin{aligned}
\mathcal{D}[R, T ; \boldsymbol{\varphi}(\boldsymbol{x})]= & \frac{1}{2} \int_{\Omega}[T(\boldsymbol{x}+\boldsymbol{u}(\boldsymbol{x}))-R(\boldsymbol{x})]^{2} d \boldsymbol{x} \\
\approx & \frac{1}{2} \int_{\Omega}\left[(T(\boldsymbol{x})-R(\boldsymbol{x}))^{2}+2(T(\boldsymbol{x})-R(\boldsymbol{x})) \nabla T(\boldsymbol{x}) \cdot \boldsymbol{u}(\boldsymbol{x})\right. \\
& \left.+(\nabla T(\boldsymbol{x}) \cdot \boldsymbol{u}(\boldsymbol{x}))^{2}+(T(\boldsymbol{x})-R(\boldsymbol{x}))\left(\boldsymbol{u}(\boldsymbol{x})^{T} \nabla^{2} T(\boldsymbol{x}) \boldsymbol{u}(\boldsymbol{x})\right)\right] d \boldsymbol{x} .
\end{aligned}
$$

The second-order derivative term $(T(\boldsymbol{x})-R(\boldsymbol{x}))\left(\boldsymbol{u}(\boldsymbol{x})^{T} \nabla^{2} T(\boldsymbol{x}) \boldsymbol{u}(\boldsymbol{x})\right)$ is omitted from the above formula, then using the first-order variation and Sherman-Morrison formula, one has the minimum point

$$
\boldsymbol{u}(\boldsymbol{x})=\frac{(T(\boldsymbol{x})-R(\boldsymbol{x})) \nabla T(\boldsymbol{x})}{|\nabla T(\boldsymbol{x})|^{2}} .
$$

Since the template image $T(\boldsymbol{x})$ in the flat region will make $|\nabla T(\boldsymbol{x})|$ small or equal to zero, resulting in the instability of the algorithm, Thirion adds a constraint term $(T(\boldsymbol{x})-R(\boldsymbol{x}))^{2}$ in the denominator to ensure the stability of the increment field, that is

$$
\boldsymbol{u}(\boldsymbol{x})=\frac{(T(\boldsymbol{x})-R(\boldsymbol{x})) \nabla T(\boldsymbol{x})}{|\nabla T(\boldsymbol{x})|^{2}+\alpha(T(\boldsymbol{x})-R(\boldsymbol{x}))^{2}},
$$

where $\nabla T(\boldsymbol{x})$ is the force of the internal boundary, and $T(\boldsymbol{x})-R(\boldsymbol{x})$ is the force of the external images.

The traditional demons only uses the gradient information of template image $T(\boldsymbol{x})$ to drive image deformation, when $\nabla T(\boldsymbol{x})$ is insufficient, it may cause the undesired registration errors. In addition, when the object deformation is large, the demons algorithm may not complete the registration requirements, even the convergence speed will be very slow. To deal with these difficulties, Wang et al. used the ideas of Newton force and reaction force to propose the active demons algorithm [49], which adds the gradient information of the reference image to obtain following formula

$$
\boldsymbol{u}(\boldsymbol{x})=\frac{(T-R) \nabla R}{(\nabla R)^{2}+\tau^{2}(T-R)^{2}}+\frac{(T-R) \nabla T}{(\nabla T)^{2}+\tau^{2}(T-R)^{2}}
$$

where the normalization factor $\tau$ is proposed by Cachier et al. [8] to adjust the force strength.

LDDMM framework. The framework of large deformation diffeomorphic metric mapping (LDDMM) can generate the diffeomorphic transformation for image registration $[4,47,45]$. The variational minimization of LDDMM framework can be defined as follow [38]:

$$
\left\{\begin{array}{l}
\min _{\boldsymbol{v}, \mathcal{U}(\cdot, 1)}\{\mathcal{J}(\boldsymbol{v}, \mathcal{U}(\cdot, 1)):=\mathcal{D}[\mathcal{U}(\cdot, 1), R]+\tau \mathcal{S}[\boldsymbol{v}]\}, \\
\text { s.t. } \mathcal{C}(\boldsymbol{v}(\boldsymbol{x}, t), \mathcal{U}(\boldsymbol{x}, t))=0, \text { on } \Omega \times[0,1]
\end{array}\right.
$$

where $\mathcal{D}$ is a similarity measure, $\mathcal{U}(\cdot, \cdot): \Omega \times[0,1] \rightarrow \mathbb{R}$ is a time series of images, $\boldsymbol{v}(\cdot, \cdot): \Omega \times[0,1] \rightarrow$ $\mathbb{R}^{d}$ is the velocity field. The regularization of the velocity field $\boldsymbol{v}(\boldsymbol{x}, t)$ can be defined by diffusion regularizer or curvature regularizer as follows

$$
\mathcal{S}^{\text {diff }}[\boldsymbol{v}]=\frac{1}{2} \int_{0}^{1} \int_{\Omega} \sum_{l=1}^{d}\left|\nabla v_{l}\right|^{2} d \boldsymbol{x} d t, \quad \mathcal{S}^{\text {curv }}[\boldsymbol{v}]=\frac{1}{2} \int_{0}^{1} \int_{\Omega} \sum_{l=1}^{d}\left|\Delta v_{l}\right|^{2} d \boldsymbol{x} d t .
$$


The constraints $\mathcal{C}(\boldsymbol{v}(\boldsymbol{x}, t), \mathcal{U}(\boldsymbol{x}, t))=0$ is given by the transport equation

$$
\mathcal{C}(\boldsymbol{v}(\boldsymbol{x}, t), \mathcal{U}(\boldsymbol{x}, t))=0 \quad \Longleftrightarrow \quad\left\{\begin{array}{l}
\partial_{t} \mathcal{U}(\boldsymbol{x}, t)+\boldsymbol{v}(\boldsymbol{x}, t) \cdot \nabla \mathcal{U}(\boldsymbol{x}, t)=0, \\
\mathcal{U}(\boldsymbol{x}, 0)=T(\boldsymbol{x})
\end{array}\right.
$$

The above intensity $\mathcal{U}(\cdot, \cdot)$ is constant along the characteristic curve $\varphi(\cdot, t)(t \in[0,1])$, i.e., $\mathcal{U}(\boldsymbol{\varphi}(\boldsymbol{x}, t), t)=T(\boldsymbol{x})$ for all $\boldsymbol{x} \in \Omega$. Hence the deformation function $\boldsymbol{\varphi}(\boldsymbol{x}, t)$ satisfies

$$
\partial_{t} \boldsymbol{\varphi}(\boldsymbol{x}, t)=\boldsymbol{v}(\boldsymbol{x}, t):=\overline{\boldsymbol{v}}(\boldsymbol{\varphi}(\boldsymbol{x}, t), t) \quad \text { and } \quad \boldsymbol{\varphi}(\boldsymbol{x}, 0)=\boldsymbol{\varphi}_{0}(\boldsymbol{x})=\boldsymbol{x} .
$$

The characteristic curve equation (2.5) can be computed by the fourth-order Runge-Kutta (RK4) scheme of ODE (2.5) as follow

$$
\boldsymbol{\varphi}_{n+1}=\operatorname{RK4}\left(\boldsymbol{\varphi}_{n}, \overline{\boldsymbol{v}}, \Delta t\right) \Leftrightarrow\left\{\begin{array}{l}
\boldsymbol{\varphi}_{n+1}=\boldsymbol{\varphi}_{n}+\frac{\Delta t}{6}\left(k_{1}+2 k_{2}+2 k_{3}+k_{4}\right) \\
k_{1}=\overline{\boldsymbol{v}}\left(\boldsymbol{\varphi}_{n}, t_{n}\right) \\
k_{2}=\overline{\boldsymbol{v}}\left(\boldsymbol{\varphi}_{n}+\frac{\Delta t}{2} k_{1}, t_{n}+\frac{\Delta t}{2}\right) \\
k_{3}=\overline{\boldsymbol{v}}\left(\boldsymbol{\varphi}_{n}+\frac{\Delta_{t} t}{2} k_{2}, t_{n}+\frac{\Delta t}{2}\right) \\
k_{4}=\overline{\boldsymbol{v}}\left(\boldsymbol{\varphi}_{n}+\Delta t k_{3}, t_{n}+\Delta t\right)
\end{array}\right.
$$

and the variational minimization (2.4) is iteratively solved to obtain the final state $\varphi_{1}(\boldsymbol{x}):=$ $\boldsymbol{\varphi}(\boldsymbol{x}, 1)$, and the other interpolation methods are also provided in an image registration toolbox FAIR [41] to obtain the deformed image $\mathcal{U}(\boldsymbol{\varphi}(\boldsymbol{x}, 1), 1)=T\left(\boldsymbol{\varphi}_{1}\right)$ [38].

H. Y. Hsiao registration. A new non-rigid registration algorithm was proposed by $\mathrm{H}$. Y. Hsiao et al. [28] which is based on the deformation method of numerical grid generation for computational fluid dynamics. The registration transformation is generated by iteratively minimizing

$$
\min _{\boldsymbol{\varphi}} \mathcal{D}_{\mathrm{SSD}}[R, T ; \boldsymbol{\varphi}]=\frac{1}{2} \int_{\Omega}(T(\boldsymbol{\varphi}(\boldsymbol{x}))-R(\boldsymbol{x}))^{2} d \boldsymbol{x},
$$

an iterative scheme of transformation $\varphi(x)$ is updated by

$$
\varphi(\boldsymbol{x})=\varphi_{\text {old }}(\boldsymbol{x})+\frac{\boldsymbol{u}(\boldsymbol{x})}{1+\operatorname{div} \boldsymbol{u}(\boldsymbol{x})} \Delta t
$$

the displacement field $\boldsymbol{u}(\boldsymbol{x})$ satisfies partial differential equation or div-curl systems

$$
\left\{\begin{array} { r l r l } 
{ \operatorname { d i v } \boldsymbol { u } ( \boldsymbol { x } ) } & { = f ( \boldsymbol { x } ) - 1 , } & { } & { \text { in } \Omega } \\
{ \operatorname { r o t } \boldsymbol { u } ( \boldsymbol { x } ) } & { = g , } & { } & { \text { in } \Omega } \\
{ \boldsymbol { u } ( \boldsymbol { x } ) } & { = \mathbf { 0 } , } & { } & { \text { on } \partial \Omega . }
\end{array} \Longrightarrow \left\{\begin{array}{rlrl}
\Delta \boldsymbol{u} & =\mathbf{F}, & & \text { in } \Omega \\
\boldsymbol{u}(\boldsymbol{x})=\mathbf{0}, & & \text { on } \partial \Omega .
\end{array}\right.\right.
$$

The another main technical achievement of this algorithm is the derivation of the gradient of $\mathcal{D}_{\text {SSD }}$ with respect to $\mathbf{F}=\left(f_{x 1}-g_{x 2}, f_{x 2}-g_{x 1}\right)$. The derivation of $\partial \mathcal{D}_{\mathrm{SSD}} / \partial \mathbf{F}$ is used as gradient to update $\mathbf{F}$ by $\mathbf{F}=\mathbf{F}_{\text {old }}+\left(\partial \mathcal{D}_{\mathrm{SSD}} / \partial \mathbf{F}\right) \Delta s$, where $\Delta s$ is the step size (also refer to [28] for more details).

3. Diffeomorphic transformation. The main use of Jacobian differential equation, which is the determinant of the Jacobian matrix of a vectorial function $\varphi(x) \in \mathbb{R}^{2}$, is found in the transformation of coordinates. While the diffeomorphism is an important topic in differential geometry, which is developed to deal with the transformation between two manifolds, in general, two different coordinate systems. 
3.1. Analysis. Dacorogna and Moser in 1990 proved the existence and regularity of diffeomorphisms based on Jacobian equation [15]. To proceed, we briefly review as follows:

Theorem 3.1 (Diffeomorphic mapping [15]). Let $\ell \geq 0$ be an integer, $0<\epsilon<1, d \geq 2$, and a bounded closed set $\Omega \subset \mathbb{R}^{d}$ have a $\mathcal{C}^{\ell+3, \epsilon}$ boundary $\partial \Omega\left(\mathcal{C}^{\ell, \epsilon}\right.$ denotes the usual Hölder spaces). $g, g_{0} \in \mathcal{C}^{\ell, \epsilon}(\Omega)$ with $g, g_{0}>0$ in $\Omega_{i n}:=\Omega / \partial \Omega$. Then there exists at least one diffeomorphism $\varphi$ with $\varphi, \varphi^{-1} \in \mathcal{C}^{\ell+1, \epsilon}\left(\Omega ; \mathbb{R}^{d}\right)$ and satisfying

$$
\left\{\begin{aligned}
g(\boldsymbol{\varphi}(\boldsymbol{x})) \operatorname{det}(\nabla \boldsymbol{\varphi}(\boldsymbol{x})) & =\lambda g_{0}(\boldsymbol{x}), & & \boldsymbol{x} \in \Omega_{\text {in }} \\
\boldsymbol{\varphi}(\boldsymbol{x}) & =\boldsymbol{x}, & & \boldsymbol{x} \in \partial \Omega
\end{aligned}\right.
$$

where $\lambda=\frac{\int_{\Omega} g(\boldsymbol{\varphi}(\boldsymbol{x})) d \boldsymbol{x}}{\int_{\Omega} g_{0}(\boldsymbol{x}) d \boldsymbol{x}}$.

Note that the above Jacobian system (3.1) may have several smooth solutions [15]. For more general measures, for instance for those with a mass density, the above problem is extended to the special setting of two mass measures $g_{0}(\cdot)$ and $g(\cdot)$ on two manifolds $\mathcal{X}$ and $\mathcal{Y}$ as finding two smooth one-to-one maps $\varphi: \mathcal{X} \rightarrow \mathcal{Y}, \varphi^{-1}: \mathcal{Y} \rightarrow \mathcal{X}$ such that

$$
\int_{\mathcal{Z}} g_{0}(\boldsymbol{x}) d \boldsymbol{x}=\int_{\mathcal{Z}} g(\boldsymbol{\varphi}(\boldsymbol{x})) \operatorname{det}(\nabla \boldsymbol{\varphi}(\boldsymbol{x})) d \boldsymbol{x}, \text { for any } \mathcal{Z} \subset \mathcal{X} .
$$

Further, the constraint (3.1) can be used to perform image registration because it does operate as an image warping method with a diffeomorphic transformation $\varphi(x)$. Intuitively, a diffeomorphic mapping $\varphi: \mathcal{X} \rightarrow \mathcal{Y}$, can be interpreted as a function moving a single point $\boldsymbol{x} \in \mathcal{X}$ from a coordinate system to another. It is also important to realize that the mass $g_{0}(\boldsymbol{x})$ defined on $\mathcal{X}$ may be a known prior but the mass $g(\boldsymbol{\varphi}(\boldsymbol{x}))$ defined on $\mathcal{Y}$ should be unknown.

REMARK 1 (Variants of Jacobian system). In the above theorem, the system (3.1) seeking a diffeomorphic mapping $\varphi$ can be rewritten as the following variants:

Case 1. assume that $g(\boldsymbol{\varphi}(\boldsymbol{x}))=1$ for all $\boldsymbol{x} \in \Omega_{\text {in }}, \int_{\Omega} g_{0}(\boldsymbol{x}) d \boldsymbol{x}=|\Omega|$, and $\lambda=1$. Thus the Jacobian equation (3.1) can be written as:

$$
\left\{\begin{aligned}
\operatorname{det}(\nabla \boldsymbol{\varphi}(\boldsymbol{x})) & =g_{0}(\boldsymbol{x}), & & \boldsymbol{x} \in \Omega_{\text {in }} \\
\boldsymbol{\varphi}(\boldsymbol{x}) & =\boldsymbol{x}, & & \boldsymbol{x} \in \partial \Omega
\end{aligned}\right.
$$

Case 2. assume that $\lambda=1, g_{0}(\boldsymbol{x})=1$ in (3.1) for all $\boldsymbol{x} \in \Omega$, and $g(\boldsymbol{\varphi}(\boldsymbol{x}))>0$, and $\int_{\Omega} g(\boldsymbol{\varphi}(\boldsymbol{x})) d \boldsymbol{x}=$ $|\Omega|$. Thus there exists at least one transformation $\varphi(x): \Omega \rightarrow \Omega$ (one-to-one and onto mapping) such that

$$
\left\{\begin{aligned}
g(\boldsymbol{\varphi}(\boldsymbol{x})) \operatorname{det}(\nabla \boldsymbol{\varphi}(\boldsymbol{x})) & =1, & & \boldsymbol{x} \in \Omega_{\text {in }} \\
\boldsymbol{\varphi}(\boldsymbol{x}) & =\boldsymbol{x}, & & \boldsymbol{x} \in \partial \Omega
\end{aligned}\right.
$$

Case 3. assume further that $f(\boldsymbol{\varphi}(\boldsymbol{x})):=1 / g(\boldsymbol{\varphi}(\boldsymbol{x}))$, the above system can be rewritten as

$$
\left\{\begin{array}{clrl}
\operatorname{det}(\nabla \boldsymbol{\varphi}(\boldsymbol{x})) & =f(\boldsymbol{\varphi}(\boldsymbol{x})), & & \boldsymbol{x} \in \Omega_{i n} \\
\boldsymbol{\varphi}(\boldsymbol{x}) & =\boldsymbol{x}, & & \boldsymbol{x} \in \partial \Omega
\end{array}\right.
$$

Since such systems are not easy to solve directly, construction of diffeomorphic mapping $\boldsymbol{\varphi}(\boldsymbol{x})$ : $\Omega \rightarrow \Omega$ of the Jacobian equation (3.2) is a very meaningful work for many image problems. Dacorogna and Moser designed a constructing method to indirectly obtain a diffeomorphic mapping $\boldsymbol{\varphi}(\boldsymbol{x})$ of (3.2) (see [15] for more details). 
To proceed, there is a need to define a suitable nonempty diffeomorphic transformation set $\operatorname{Diff}_{\varphi}^{f(\varphi)}(\Omega)$ by the solution of the differential system (3.3) as

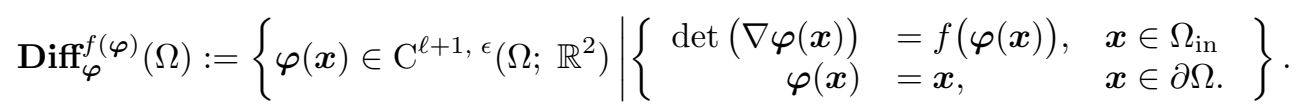

Without loss of generality, we restrict our attention to $\ell=1$ in the following part.

3.2. Construction. Before seeking a diffeomorphic transformation $\boldsymbol{\varphi}(\boldsymbol{x})$ in (3.3), we first analyze a constructing method of the time-dependent diffeomorphic solution $\boldsymbol{\phi}(\boldsymbol{x}, t)$ (corresponding to the coordinate system at time $t$ ) that satisfies the time-sequence Jacobian equation defined by

$$
\left\{\begin{aligned}
g(\boldsymbol{\phi}(\boldsymbol{x}, t)) \operatorname{det} \nabla \boldsymbol{\phi}(\boldsymbol{x}, t) & =g(\boldsymbol{\phi}(\boldsymbol{x}, 0)) \operatorname{det} \nabla \boldsymbol{\phi}(\boldsymbol{x}, 0)=g_{0}(\boldsymbol{x}), & & \boldsymbol{x} \in \Omega_{\text {in }} \\
\boldsymbol{\phi}(\boldsymbol{x}, t) & =\boldsymbol{x}, & & \boldsymbol{x} \in \partial \Omega \\
\boldsymbol{\phi}(\boldsymbol{x}, 0) & =\boldsymbol{x}, & & \boldsymbol{x} \in \Omega
\end{aligned}\right.
$$

for any $t \in[0,1]$, where $\frac{\int_{\Omega} g(\phi(\boldsymbol{x}, t)) d \boldsymbol{x}}{\int_{\Omega} g_{0}(\boldsymbol{x}) d \boldsymbol{x}}=1$. Especially if taking $g(\boldsymbol{\phi}(\boldsymbol{x}, t))=1 / f(\boldsymbol{\phi}(\boldsymbol{x}, t))>0$, one easily has

$$
\left\{\begin{aligned}
\operatorname{det} \nabla \phi(\boldsymbol{x}, t) & =\frac{f(\boldsymbol{\phi ( x , t ) )}}{f(\boldsymbol{x})}, & & \boldsymbol{x} \in \Omega_{\text {in }} \\
\phi(\boldsymbol{x}, t) & =\boldsymbol{x}, & & \boldsymbol{x} \in \partial \Omega \\
\boldsymbol{\phi}(\boldsymbol{x}, 0) & =\boldsymbol{x}, & & \boldsymbol{x} \in \Omega .
\end{aligned}\right.
$$

The following technical Lemma is useful for understanding the construction method solving time-dependent diffeomorphism $\boldsymbol{\phi}(\boldsymbol{x}, t)$ in (3.6):

Lemma 3.2 (Abel-Jacobi-Liouville Identity [36]). Let $\Phi(t)$ be a $n \times n$ matrix function where each element is differentiable on $t \in[0,1]$. If $\frac{d \Phi(t)}{d t}=A(t) \Phi(t)$ where $A(t)$ is a $n \times n$ matrix, then one has $\frac{d}{d t} \operatorname{det} \Phi(t)=\operatorname{Tr} A(t) \operatorname{det} \Phi(t)$, where $\boldsymbol{T r} A(t)$ is the trace of $A(t)$.

3.2.1. Dynamical system. Inspired by the knowledge in [15] and the work in [35], the previous literature (see Theorem 3.1) shows that there exists a diffeomorphic transformation $\boldsymbol{\phi}(\boldsymbol{x}, t) \in \mathcal{C}^{\ell+1, \epsilon}(\Omega,[0,1])$ of system (3.6) with $f(\boldsymbol{x})=1$, for which we can find $\boldsymbol{\varphi}(\boldsymbol{x})=\boldsymbol{\phi}(\boldsymbol{x}, 1)$ satisfying (3.3). To construct such diffeomorphic transformation $\boldsymbol{\phi}(\boldsymbol{x}, t)$, we consider the following continuous evolution system

$$
\frac{d}{d t} \phi(x, t)=\boldsymbol{v}(\phi(x, t), t)
$$

The state $\phi(\boldsymbol{x}, t)$ of the above dynamical system evolves through continuous time $t \in[0,1]$, such time sequence $\boldsymbol{\phi}(\boldsymbol{x}, t)$ can be seen as a coordinate system that moves through the state space. The evolution rule will specify how this coordinate system $\phi(\boldsymbol{x}, t)$ moves by designing its velocity field $\boldsymbol{v}(\boldsymbol{\phi}(\boldsymbol{x}, t), t)$. In this case, starting with an initial state (coordinate system) $\boldsymbol{\phi}(\boldsymbol{x}, 0)=\boldsymbol{x}$ at time $t=0$, the trajectory $\phi(\boldsymbol{x}, t)$ of all future times will be a new state through state space.

We also notice that the velocity field $\boldsymbol{v}(\boldsymbol{\phi}(\boldsymbol{x}, t), t)$ from interactions between different variants (components) of $\boldsymbol{\phi}(\boldsymbol{x}, t)$ cannot be explained in terms of the individual properties of these variants alone. Although $\boldsymbol{v}(\boldsymbol{\phi}(\boldsymbol{x}, t), t)$ cannot be measured in an experiment directly, it can in principle be obtained from the prior knowledge of control increment $\boldsymbol{u}(\boldsymbol{\phi}(\boldsymbol{x}, t))$ of the state $\phi(\boldsymbol{x}, t)$ in the process of creating a dynamical system.

To illustrate the idea of dynamical system solving (3.3) or (3.6), the velocity field can be analytically designed as

$$
\boldsymbol{v}(\phi(\boldsymbol{x}, t), t):=\frac{\boldsymbol{u}(\boldsymbol{\phi}(\boldsymbol{x}, t))}{h(\phi(\boldsymbol{x}, t), t)},
$$


where a homotopy composite function $h(\boldsymbol{\phi}(\boldsymbol{x}, t), t)$ will be constructed in Section 3.2.4.

3.2.2. Control increment system. One of main ingredients for constructing the diffeomorphic solution $\boldsymbol{\varphi}(\boldsymbol{x})=\boldsymbol{\phi}(\boldsymbol{x}, 1)$ of system (3.3) is to determine control increment $\boldsymbol{u}(\boldsymbol{\phi}(\boldsymbol{x}, t))$ by manually choosing the composite function $h(\boldsymbol{\phi}(\boldsymbol{x}, t), t)$. Now let us look at how we may apply (3.7) and (3.8) to exploit $\boldsymbol{u}(\boldsymbol{\phi}(\boldsymbol{x}, t))$. To express this discussion in mathematical terms, we define

$$
H(\boldsymbol{\phi}(\boldsymbol{x}, t), t)=\operatorname{det} \nabla \boldsymbol{\phi}(\boldsymbol{x}, t) h(\boldsymbol{\phi}(\boldsymbol{x}, t), t) .
$$

LEMMA 3.3. Let $h(\boldsymbol{\phi}(\boldsymbol{x}, t), t)>0$ be a homotopy composite function with $h(\boldsymbol{\phi}(\boldsymbol{x}, 0), 0)=$ $h(\boldsymbol{x}, 0)$. If the control increment $\boldsymbol{u}(\boldsymbol{\phi}(\boldsymbol{x}, t))$ in (3.8) is a solution of the following PDE system

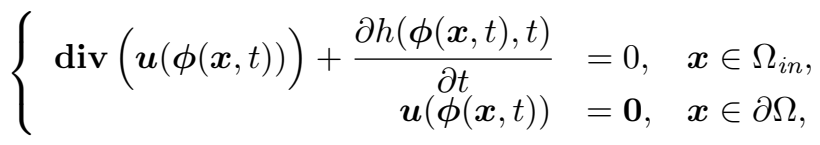

then the solution $\boldsymbol{\phi}(\boldsymbol{x}, t)$ of the equation (3.7) with $\boldsymbol{v}(\boldsymbol{\phi}(\boldsymbol{x}, t), t):=\frac{\boldsymbol{u}(\boldsymbol{\phi}(\boldsymbol{x}, t))}{h(\boldsymbol{\phi}(\boldsymbol{x}, t), t)}$ satisfies

$$
\operatorname{det} \nabla \boldsymbol{\phi}(\boldsymbol{x}, t)=\frac{h(\boldsymbol{x}, 0)}{h(\boldsymbol{\phi}(\boldsymbol{x}, t), t)}, \text { for all } t \in(0,1] .
$$

Proof. Let us consider the gradient form of the equation (3.7) as

$$
\nabla\left(\frac{d}{d t} \phi(\boldsymbol{x}, t)\right)=\frac{d}{d t} \nabla \boldsymbol{\phi}(\boldsymbol{x}, t)=\nabla \boldsymbol{v}(\boldsymbol{\phi}(\boldsymbol{x}, t), t) \nabla \boldsymbol{\phi}(\boldsymbol{x}, t)
$$

which can be written by using Lemma 3.2 as

$$
\frac{d}{d t} \operatorname{det} \nabla \phi(\boldsymbol{x}, t)=\operatorname{Tr}(\nabla \boldsymbol{v}(\boldsymbol{\phi}(\boldsymbol{x}, t), t)) \operatorname{det} \nabla \boldsymbol{\phi}(\boldsymbol{x}, t)=\operatorname{div}(\boldsymbol{v}(\boldsymbol{\phi}(\boldsymbol{x}, t), t)) \operatorname{det} \nabla \boldsymbol{\phi}(\boldsymbol{x}, t) .
$$

Further from (3.8), we easily obtain

$$
\boldsymbol{u}(\phi(\boldsymbol{x}, t))=\boldsymbol{v}(\boldsymbol{\phi}(\boldsymbol{x}, t), t) h(\phi(\boldsymbol{x}, t), t)
$$

and

$$
\operatorname{div}(\boldsymbol{v}(\phi(x, t), t)) h(\phi(x, t), t)=\operatorname{div}(\boldsymbol{u}(\phi(x, t)))-\boldsymbol{v}(\phi(x, t), t) \cdot \nabla h(\phi(x, t), t) .
$$

Therefore, by rearranging this expression, we obtain the following derivative formula for estimating the properties of the function $H(\phi(x, t), t)$ :

$$
\begin{aligned}
& \frac{d}{d t} H(\boldsymbol{\phi}(\boldsymbol{x}, t), t)= h(\boldsymbol{\phi}(\boldsymbol{x}, t), t) \frac{d}{d t} \operatorname{det} \nabla \boldsymbol{\phi}(\boldsymbol{x}, t) \\
&+\operatorname{det} \nabla \boldsymbol{\phi}(\boldsymbol{x}, t)\left(\frac{\partial h(\boldsymbol{\phi}(\boldsymbol{x}, t), t)}{\partial t}+\nabla h(\boldsymbol{\phi}(\boldsymbol{x}, t), t) \cdot \frac{d}{d t} \boldsymbol{\phi}(\boldsymbol{x}, t)\right) \\
&= \operatorname{det} \nabla \boldsymbol{\phi}(\boldsymbol{x}, t)(\operatorname{div}(\boldsymbol{v}(\boldsymbol{\phi}(\boldsymbol{x}, t), t)) h(\boldsymbol{\phi}(\boldsymbol{x}, t), t) \\
&+\left.\frac{\partial h(\boldsymbol{\phi}(\boldsymbol{x}, t), t)}{\partial t}+\nabla h(\boldsymbol{\phi}(\boldsymbol{x}, t), t) \cdot \frac{d}{d t} \boldsymbol{\phi}(\boldsymbol{x}, t)\right) \\
&=\operatorname{det} \nabla \boldsymbol{\phi}(\boldsymbol{x}, t)\left(\operatorname{div}(\boldsymbol{u}(\boldsymbol{\phi}(\boldsymbol{x}, t)))+\frac{\partial h(\boldsymbol{\phi}(\boldsymbol{x}, t), t)}{\partial t}\right) .
\end{aligned}
$$


If letting

$$
\operatorname{div}(\boldsymbol{u}(\boldsymbol{\phi}(\boldsymbol{x}, t)))+\frac{\partial h(\phi(x, t), t)}{\partial t}=0
$$

it is easy to check that $\frac{d}{d t} H(\phi(\boldsymbol{x}, t), t)=0$ for all $t \in(0,1]$, hence we obtain

$$
h(\boldsymbol{x}, 0)=\operatorname{det} \nabla \boldsymbol{\phi}(\boldsymbol{x}, 0) h(\boldsymbol{x}, 0)=H(\boldsymbol{x}, 0)=H(\boldsymbol{\phi}(\boldsymbol{x}, t), t)=\operatorname{det} \nabla \boldsymbol{\phi}(\boldsymbol{x}, t) h(\boldsymbol{\phi}(\boldsymbol{x}, t), t),
$$

i.e.,

$$
\operatorname{det} \nabla \boldsymbol{\phi}(\boldsymbol{x}, t)=\frac{h(\boldsymbol{x}, 0)}{h(\boldsymbol{\phi}(\boldsymbol{x}, t), t)}, \text { for all } t \in(0,1]
$$

According to the above construction, if we consider the boundary condition $\boldsymbol{u}(\boldsymbol{\phi}(\boldsymbol{x}, t))=\mathbf{0}$ for all $\boldsymbol{x} \in \partial \Omega$, thus the control increment $\boldsymbol{u}(\boldsymbol{\phi}(\boldsymbol{x}, t))$ of the state $\boldsymbol{\phi}(\boldsymbol{x}, t)$ in (3.8) is defined as a non-uniqueness solution of the following PDE system:

$$
\left\{\begin{array}{rll}
\operatorname{div}(\boldsymbol{u}(\boldsymbol{\phi}(\boldsymbol{x}, t)))+\frac{\partial h(\boldsymbol{\phi}(\boldsymbol{x}, t), t)}{\partial t} & =0, & \boldsymbol{x} \in \Omega_{\mathrm{in}} \\
\boldsymbol{u}(\boldsymbol{\phi}(\boldsymbol{x}, t)) & =\mathbf{0}, & \boldsymbol{x} \in \partial \Omega
\end{array}\right.
$$

which proves the assertion.

We remark that similar construction of the control increment $\boldsymbol{u}(\boldsymbol{\phi}(\boldsymbol{x}, t))$ can be found in $[15,35]$.

3.2.3. Boundary condition. Next we analyze that the solution $\phi(\boldsymbol{x}, 1)$ in $(3.7)$ satisfies the boundary condition of (3.3), i.e., $\phi(\boldsymbol{x}, 1)=\boldsymbol{x}$ when $\boldsymbol{x} \in \partial \Omega$. Since $\boldsymbol{u}(\boldsymbol{\phi}(\boldsymbol{x}, t))=\mathbf{0}$ for all $\boldsymbol{x} \in \partial \Omega$ and $t \in[0,1]$, (3.7) can be simplified as:

$$
\frac{d}{d t} \boldsymbol{\phi}(\boldsymbol{x}, t)=\boldsymbol{v}(\boldsymbol{\phi}(\boldsymbol{x}, t), t)=\mathbf{0}, \quad \boldsymbol{x} \in \partial \Omega .
$$

Hence $\boldsymbol{\phi}(\boldsymbol{x}, t)$ is independent of $t$ on the boundary $\partial \Omega$, it means that $\boldsymbol{\phi}(\boldsymbol{x}, 1)=\boldsymbol{\phi}(\boldsymbol{x}, t)=\boldsymbol{\phi}(\boldsymbol{x}, 0)=$ $\boldsymbol{x}$ for any $\boldsymbol{x} \in \partial \Omega$. Furthermore, combining with (3.11), one has

$$
\left\{\begin{aligned}
\operatorname{det} \nabla \boldsymbol{\phi}(\boldsymbol{x}, t) & =\frac{h(\boldsymbol{x}, 0)}{h(\boldsymbol{\phi}(\boldsymbol{x}, t), t)}, & & \boldsymbol{x} \in \Omega_{\text {in }} \\
\boldsymbol{\phi}(\boldsymbol{x}, t) & =\boldsymbol{x}, & & \boldsymbol{x} \in \partial \Omega
\end{aligned}\right.
$$

for any $t \in(0,1]$.

REMARK 2. Especially if one takes $t=1$, then the solution $\boldsymbol{\varphi}(\boldsymbol{x})=\boldsymbol{\phi}(\boldsymbol{x}, 1)$ obtained by the above construction method satisfies the differential system

$$
\left\{\begin{aligned}
\operatorname{det}(\nabla \boldsymbol{\varphi}(\boldsymbol{x})) & =\frac{h(\boldsymbol{x}, 0)}{h(\boldsymbol{\varphi}(\boldsymbol{x}), 1)}, & & \boldsymbol{x} \in \Omega_{i n}, \\
\boldsymbol{\varphi}(\boldsymbol{x}) & =\boldsymbol{x}, & & \boldsymbol{x} \in \partial \Omega .
\end{aligned}\right.
$$

3.2.4. Choice of path following composite function. Path following method, also called imbedding and homotopy continuation method, was first applied to the solution of separation models involving large numbers of nonlinear equations [44], which provides an useful approach to find the zeros of nonlinear function $f: \mathbb{R}^{n} \rightarrow \mathbb{R}^{n}$ in a globally convergent way.

As has been discussed, $g_{0}(\boldsymbol{x})$ and $g(\boldsymbol{\varphi}(\boldsymbol{x}))$ are two mass measures on two manifolds $\mathcal{X}$ and $\mathcal{Y}$, especially in $\boldsymbol{x}$ - and $\boldsymbol{\varphi}(\boldsymbol{x})$-coordinate systems. The purpose of considering the path following in 


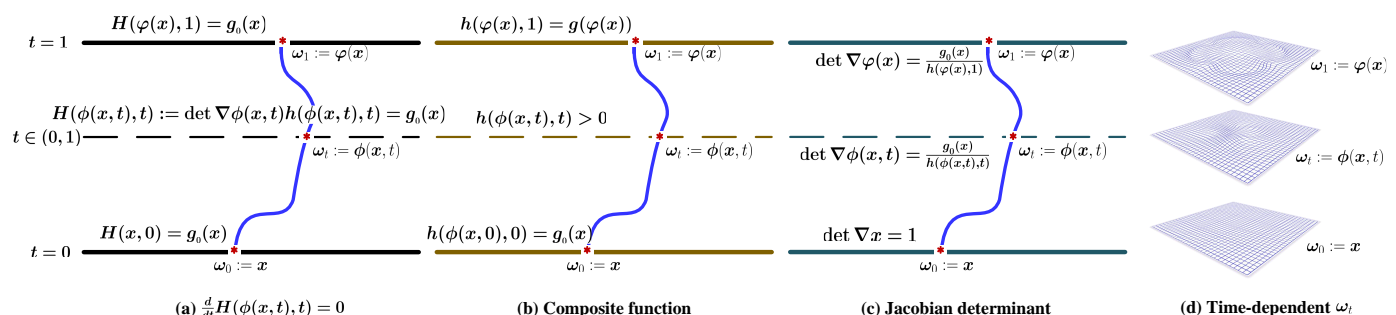

FIG. 1. Time-sequence transformation $\boldsymbol{\omega}_{t}$, composition function and time-dependent Jacobian determinant.

this work is to build a smooth path between the beginning solution $\phi(x, 0)=\boldsymbol{x}$ of (3.6) at time $t=0$ and the ending target solution $\phi(x, 1)=\varphi(x)$ of (3.6) at time $t=1$ (see blue curve in Fig.1). This means that it gradually deforms this initial transformation $\boldsymbol{x}$ (coordinate system at time $t=0)$ into one helpful deformation field $\boldsymbol{\phi}(\boldsymbol{x}, t)$ of (3.6) as time $t \in[0,1]$, and eventually ends with a perfect registration $T(\boldsymbol{\varphi}(\boldsymbol{x})) \approx R(\boldsymbol{x})$ between the template $T(\boldsymbol{x})$ and the reference $R(\boldsymbol{x})$.

To obtain system (3.11), the design of $H(\boldsymbol{\phi}(\boldsymbol{x}, t), t)=\operatorname{det} \nabla \boldsymbol{\phi}(\boldsymbol{x}, t) h(\boldsymbol{\phi}(\boldsymbol{x}, t), t)$ is one of the most important steps, where the function $H(\boldsymbol{\phi}(\boldsymbol{x}, t), t)$ satisfies $\frac{d}{d t} H(\boldsymbol{\phi}(\boldsymbol{x}, t), t)=0$ for all $t \in(0,1]$, i.e.,

$$
g_{0}(\boldsymbol{x}):=h(\boldsymbol{x}, 0)=H(\boldsymbol{x}, 0)=H(\boldsymbol{\phi}(\boldsymbol{x}, t), t)=\operatorname{det} \nabla \boldsymbol{\phi}(\boldsymbol{x}, t) h(\boldsymbol{\phi}(\boldsymbol{x}, t), t)
$$

if (3.10) holds (see Fig.1(a)).

As has been seen from (3.7) and (3.10), the function $h(\boldsymbol{\phi}(\boldsymbol{x}, t), t)$ selectively determines the control increment $\boldsymbol{u}(\phi(\boldsymbol{x}, t))$ and the velocity field $\boldsymbol{v}(\phi(\boldsymbol{x}, t), t)$ of the evolving deformation $\boldsymbol{\phi}(\boldsymbol{x}, t)$. Next we follow the discussion in [1] to construct a composite function $h(\boldsymbol{\phi}(\boldsymbol{x}, t), t)$ that allows to use different homotopy variants to serve the objective of continuously deforming an origin mass function $g_{0}(\boldsymbol{x})$ at $t=0$ into a target mass function $g(\boldsymbol{\varphi}(\boldsymbol{x}))$ at $t=1$ (see Fig. 1(b)). Here the set of admissible path following composite functions is defined by

$$
\begin{aligned}
& \mathcal{H}_{g, \boldsymbol{\phi}}(\Omega,[0,1]):=\left\{h(\boldsymbol{\phi}(\boldsymbol{x}, t), t) \in \mathcal{C}^{\ell}(\Omega,[0,1]) \mid \boldsymbol{\phi}(\boldsymbol{x}, t) \in \mathcal{C}^{\ell+1, \epsilon}(\Omega,[0,1]),\right. \\
& h(\boldsymbol{\phi}(\boldsymbol{x}, t), t)>0 \text { and } \frac{\int_{\Omega} h(\boldsymbol{\phi}(\boldsymbol{x}, t), t) d \boldsymbol{x}}{\int_{\Omega} g_{0}(\boldsymbol{x}) d \boldsymbol{x}}=1, \forall t \in[0,1], \\
&\left.h(\boldsymbol{\phi}(\boldsymbol{x}, 0), 0)=g_{0}(\boldsymbol{x}) \text { and } h(\boldsymbol{\phi}(\boldsymbol{x}, 1), 1)=g(\boldsymbol{\varphi}(\boldsymbol{x})) \text { on } \Omega\right\},
\end{aligned}
$$

then we list several composite functions that allows to be used in Section 3.2 as follows

P1: $h(\boldsymbol{\phi}(\boldsymbol{x}, t), t)=(1-t) g_{0}(\boldsymbol{x})+t g(\boldsymbol{\phi}(\boldsymbol{x}, t))$, for all $t \in[0,1]$;

P2: $h(\boldsymbol{\phi}(\boldsymbol{x}, t), t)=g_{0}(\boldsymbol{x}) e^{t \ln \frac{g(\boldsymbol{\phi}(\boldsymbol{x}, t))}{g_{0}(\boldsymbol{x})}}$, for all $t \in[0,1]$;

P3: $h(\phi(\boldsymbol{x}, t), t)=(1-t) \operatorname{det}(\nabla \boldsymbol{\phi}(\boldsymbol{x}, t))+t g(\boldsymbol{\phi}(\boldsymbol{x}, t))$, for all $t \in[0,1]$;

P4: $h(\phi(\boldsymbol{x}, t), t)=g(\boldsymbol{\phi}(\boldsymbol{x}, t))$, for all $t \in[0,1]$.

For discrete setting, the mapping $\boldsymbol{\phi}(\boldsymbol{x}, t)$ consists simply in moving the positions $\boldsymbol{x}_{i}=\left(x_{1}^{i}, x_{2}^{i}\right)$ of all the grid points to new positions $\phi\left(\boldsymbol{x}_{i}, t\right)$, for instance for those with a grid quality measure $g(\boldsymbol{\phi}(\boldsymbol{x}, t))$, the notion of $\boldsymbol{\phi}(\boldsymbol{x}, t)$ plays a fundamental to describe spatial modifications for image registration. The mass measure $g(\boldsymbol{\phi}(\boldsymbol{x}, t))$ guarantees that $\boldsymbol{\phi}(\boldsymbol{x}, t)$ is diffeomorphic, unfortunately it is unknown and depends on the moving image $T(\boldsymbol{\phi}(\boldsymbol{x}, t))$ and the reference $R(\boldsymbol{x})$, also depends on $\phi(x, t)$ that be partly driven by SSD for image registration. Hence we consider the dirac delta 
approximation defined by

$$
\begin{aligned}
g(\boldsymbol{\phi}(\boldsymbol{x}, t))=\int_{\mathbb{R}^{2}} g(\boldsymbol{y}) \delta(\phi(\boldsymbol{x}, t)-\boldsymbol{y}) d \boldsymbol{y} & =\lim _{\sigma \rightarrow 0} \frac{1}{2 \pi \sigma^{2}} \int_{\mathbb{R}^{2}} g(\boldsymbol{y}) \exp \left(-\frac{\|\boldsymbol{y}-\boldsymbol{\phi}(\boldsymbol{x}, t)\|^{2}}{2 \sigma^{2}}\right) d \boldsymbol{y} \\
& \approx \frac{1}{2 \pi \sigma_{\epsilon}^{2}} \int_{\Omega} g(\boldsymbol{y}) \exp \left(-\frac{\|\boldsymbol{y}-\boldsymbol{\phi}(\boldsymbol{x}, t)\|^{2}}{2 \sigma_{\epsilon}^{2}}\right) d \boldsymbol{y}
\end{aligned}
$$

where $g(\boldsymbol{y}):=g_{0}(\boldsymbol{x})=1$ is an uniform mass density of the point $\boldsymbol{y}$ in origin $\boldsymbol{y}$-coordinate system for our registration problem at time $t=0$.

In our work, an appropriate composite function $h(\boldsymbol{\phi}(\boldsymbol{x}, t), t)$ will be chose to exploit the control increment $\boldsymbol{u}(\boldsymbol{\phi}(\boldsymbol{x}, t))$ of the diffusion equation (3.10), and such $\boldsymbol{u}(\boldsymbol{\phi}(\boldsymbol{x}, t))$ is also required to minimize registration energy. Hence $\boldsymbol{\phi}(\boldsymbol{x}, t)$ satisfies the diffeomorphic system (3.11) for any time $t \in[0,1]$.

3.3. Implementation. We can deduce the implementation, following reasoning similar to that in [12]. Then the overall constructing process based on a dynamical system may be summarized as follows.

Step 1. Designing manually a homotopy composite function $h(\boldsymbol{\phi}(\boldsymbol{x}, t), t) \in \mathcal{H}_{g, \boldsymbol{\phi}}(\Omega,[0,1])$ such that

$$
h(\boldsymbol{\phi}(\boldsymbol{x}, 0), 0)=g_{0}(\boldsymbol{x}) \text { and } h(\boldsymbol{\phi}(\boldsymbol{x}, 1), 1)=g(\boldsymbol{\phi}(\boldsymbol{x}, 1))=g(\boldsymbol{\varphi}(\boldsymbol{x})) \text { for all } \boldsymbol{x} \in \Omega \text {. }
$$

Step 2. Solving $\boldsymbol{u}(\boldsymbol{\phi}(\boldsymbol{x}, t)): \Omega \rightarrow \mathbb{R}^{2}$, such that

$$
\left\{\begin{aligned}
\operatorname{div} \boldsymbol{u}(\boldsymbol{\phi}(\boldsymbol{x}, t)) & =-\frac{\partial h(\boldsymbol{\phi}(\boldsymbol{x}, t), t)}{\partial t}, & & \boldsymbol{x} \in \Omega_{\mathrm{in}} \\
\boldsymbol{u}(\boldsymbol{\phi}(\boldsymbol{x}, t)) & =\mathbf{0}, & & \boldsymbol{x} \in \partial \Omega
\end{aligned}\right.
$$

where $\phi(\boldsymbol{x}, t) \in \Omega$ for all $t \in[0,1]$.

Step 3. Solving $\boldsymbol{\phi}(\boldsymbol{x}, t)$ from the dynamical system

$$
\left\{\begin{aligned}
\frac{d}{d t} \boldsymbol{\phi}(\boldsymbol{x}, t) & =\boldsymbol{v}(\boldsymbol{\phi}(\boldsymbol{x}, t), t):=\frac{\boldsymbol{u}(\boldsymbol{\phi}(\boldsymbol{x}, t))}{h(\phi(\boldsymbol{x}, t), t)}, & & \boldsymbol{x} \in \Omega \\
\phi(\boldsymbol{x}, 0) & =\boldsymbol{x}, & & \boldsymbol{x} \in \Omega .
\end{aligned}\right.
$$

A solution $\boldsymbol{\phi}(\boldsymbol{x}, 1)$ of (3.18) satisfies the differential system (3.3), therefore we can solve Step 2 and Step 3 alternately to obtain the solution of the differential system (3.3).

The dynamical system (3.18) is an ODE system that can be integrated numerically using a high-order difference scheme. In image registration, the transformation $\boldsymbol{\phi}\left(\boldsymbol{x}, t_{k+1}\right)$ at time step $t_{k+1}$ is updated from the current transformation $\phi\left(\boldsymbol{x}, t_{k}\right)$ by solving equation (3.18) with the RK4 difference scheme (2.6), namely

$$
\phi\left(\boldsymbol{x}, t_{k+1}\right)=\mathbf{R K} 4\left(\phi\left(\boldsymbol{x}, t_{k}\right), \boldsymbol{v}, \Delta t\right) .
$$

We also refer readers to literatures [29, 38, 40, 41] for more details. Therefore, the solution $\boldsymbol{\varphi}(\boldsymbol{x})=\boldsymbol{\phi}(\boldsymbol{x}, 1)$ obtained by the above construction method satisfies the differential system

$$
\left\{\begin{aligned}
\operatorname{det}(\nabla \boldsymbol{\varphi}(\boldsymbol{x})) & =\frac{h(\boldsymbol{x}, 0)}{h(\boldsymbol{\varphi}(\boldsymbol{x}), 1)}, & & \boldsymbol{x} \in \Omega_{\text {in }} \\
\boldsymbol{\varphi}(\boldsymbol{x}) & =\boldsymbol{x}, & & \boldsymbol{x} \in \partial \Omega .
\end{aligned}\right.
$$


3.4. Control increment in diffeomorphic image registration. Although there are many methods for solving the above control increment PDE system (3.17) with non-unique solutions, it is impossible to find useful formulas for the close-form solutions of most. To look for numerical approximations $\boldsymbol{u}(\phi(\boldsymbol{x}, t))$ for diffeomorphic image registration, it is useful to add an optimization that guarantees suitable increment $\boldsymbol{u}(\phi(\boldsymbol{x}, t))$ of the diffeomorphic solution $\boldsymbol{\phi}(\boldsymbol{x}, t)$. Therefore, the time sequential minimization problem

$$
\min _{\boldsymbol{u}(\boldsymbol{\phi}(\cdot, t))}\left\{\mathcal{E}_{0}^{t}(\boldsymbol{u}):=\mathcal{D}[R, T ; \phi(\cdot, t)+\alpha(t) \boldsymbol{u}(\boldsymbol{\phi}(\cdot, t))]+\tau \mathcal{S}[\boldsymbol{u}(\boldsymbol{\phi}(\cdot, t))]\right\}
$$

is imposed as a new restriction for $\boldsymbol{u}(\boldsymbol{\phi}(\boldsymbol{x}, t))$, together with the control increment system (3.17) (A feasible set), to ensure the existence of an unique increment $\boldsymbol{u}(\boldsymbol{\phi}(\boldsymbol{x}, t))$. Here $\boldsymbol{\phi}(\boldsymbol{x}, t)$ is the state at time $t$, especially if $t$ is fixed, the state $\phi(\boldsymbol{x}, t)$ can be explicitly updated. Moreover, $\boldsymbol{u}$ not only satisfies the PDE system (3.17) but also ensures that the deformation field $\boldsymbol{\phi}(\boldsymbol{x}, t)$ has good smoothness and diffeomorphism. Here $\mathcal{S}[\boldsymbol{u}(\phi(\cdot, t))]$ is the regularizer, $\tau>0$ is the regularization parameter. Hence $T(\boldsymbol{\phi}(\boldsymbol{x}, t))$ is spatially aligned with $R(x)$.

REMARK 3. As we can observe, a well-posed $\boldsymbol{u}(\boldsymbol{\phi}(\boldsymbol{x}, t))$ in image registration has been obtained in case of minimizing local $\mathcal{E}_{0}^{t}(\boldsymbol{u})$ (at time $t$ ). Although the first-order update of $\boldsymbol{\phi}(\boldsymbol{x}, t)$ is employed in energy $\mathcal{E}_{0}^{t}(\boldsymbol{u})$, it essentially admits a solution $\boldsymbol{u}(\boldsymbol{\phi}(\boldsymbol{x}, t))$ satisfying the constraint (3.17). It is well known that the $\boldsymbol{R} \boldsymbol{K} 4$ discretization of ODE (3.18) is a stable higher-order accurate difference scheme, which has been widely used in image registration for obtaining a diffeomorphic solution $[38,41]$.

4. The proposed optimal control relaxation model for diffeomorphic registration. In this section, we aim to present an optimal control system modeling image registration, which can help to get a diffeomorphic deformation.

The diffeomorphic image registration is, in the space of diffeomorphism deformation field $\operatorname{Diff}_{\varphi}^{f(\boldsymbol{\varphi})}(\Omega)\left(f(\boldsymbol{\varphi})>0\right.$ and $\left.\int_{\Omega} 1 / f(\boldsymbol{\varphi}(\boldsymbol{x})) d \boldsymbol{x}=|\Omega|\right)$, to seek an optimal solution $\boldsymbol{\varphi}$ with some regularity $\mathcal{S}[\varphi]$ such that $\mathcal{D}[R, T ; \varphi]$ is as small as possible. Here we consider diffeomorphic image registration model as a constrained optimization problem

$$
\min _{\varphi \in \operatorname{Diff}_{\varphi}^{f(\varphi)}(\Omega)}\{\mathcal{E}(\varphi):=\mathcal{D}[R, T ; \varphi]+\tau \mathcal{S}[\varphi]\}
$$

where $\operatorname{Diff}_{\varphi}^{f(\varphi)}(\Omega)$ is defined by (3.4). Usually the regularization term $\mathcal{S}[\boldsymbol{\varphi}]$ can be elastic modulus [6], diffusion modulus [16] or curvature modulus [17], etc.

DeFinition 4.1. For a function $\boldsymbol{f}: \Omega \rightarrow \mathbb{R}^{2}$, assume that

$$
[\boldsymbol{f}]_{\Omega}^{k, \epsilon}=\sup _{\boldsymbol{x}, \boldsymbol{y} \in \Omega, \boldsymbol{x} \neq \boldsymbol{y}} \frac{\left|\partial^{k} \boldsymbol{f}(\boldsymbol{x})-\partial^{k} \boldsymbol{f}(\boldsymbol{y})\right|}{|\boldsymbol{x}-\boldsymbol{y}|^{\epsilon}}<+\infty, \quad k=0,1, \ldots, \ell+1 .
$$

$A$ bounded Hölder subset $\mathbf{H B}^{\ell+1, \epsilon}$ is defined as

$$
\begin{aligned}
& \mathbf{H B}^{\ell+1, \epsilon}:=\left\{\boldsymbol{f} \in \mathcal{C}_{0}^{\ell+1, \epsilon}(\Omega,[0,1])\right.: \exists \mathcal{L}>0 \text { such that } \\
&\left.\|\boldsymbol{f}\|_{\infty} \leq 1 \text { and }[\boldsymbol{f}]_{\Omega}^{k, \epsilon} \leq \mathcal{L}, \forall k=0, \ldots, \ell+1\right\} .
\end{aligned}
$$

In the previous Section, we have known that the differential system (3.3) exists at least one diffeomorphic solution $\varphi(x)$, so the solution set $\operatorname{Diff}_{\varphi}^{f(\varphi)}(\Omega)$ is not empty. A method to solve $\varphi \in \operatorname{Diff}_{\varphi}^{f(\varphi)}(\Omega)$ is constructed, and one of the important steps is to solve (3.17). We denote the 
constraint set $\mathbf{D} \mathbf{C}_{h\left(\phi_{t}\right)}(\Omega)$ as the solution set of differential system

$$
\left\{\begin{aligned}
\operatorname{div} \boldsymbol{u}(\boldsymbol{\phi}(\boldsymbol{x}, t))= & -\frac{\partial h(\boldsymbol{\phi}(\boldsymbol{x}, t), t)}{\partial t}, & & \boldsymbol{x} \in \Omega_{\mathrm{in}}, \\
\boldsymbol{u}(\boldsymbol{\phi}(\boldsymbol{x}, t))= & \mathbf{0}, & & \boldsymbol{x} \in \partial \Omega, \\
\boldsymbol{u}(\boldsymbol{\phi}(\boldsymbol{x}, t)) & \in \mathbf{H B}^{\ell+1, \epsilon} \subset \mathcal{C}_{0}^{\ell+1, \epsilon}(\Omega,[0,1]) & & \text { and } \boldsymbol{\phi}(\boldsymbol{x}, t) \in \mathcal{C}^{\ell+1, \epsilon}(\Omega,[0,1])
\end{aligned}\right.
$$

Further we employ a new coordinate system $\boldsymbol{\omega}_{t}$ to represent the transformed grid $\phi(\boldsymbol{x}, t)$ at time $t$, hence $\mathbf{D} \mathbf{C}_{h\left(\boldsymbol{\omega}_{t}\right)}(\Omega)$ replacing $\mathbf{D} \mathbf{C}_{h\left(\phi_{t}\right)}(\Omega)$ denotes the solution set of differential system

$$
\left\{\begin{array}{rlrl}
\operatorname{div} \boldsymbol{u}\left(\boldsymbol{\omega}_{t}\right) & =-\frac{\partial h\left(\boldsymbol{\omega}_{t}, t\right)}{\partial t}, & & \boldsymbol{\omega}_{t} \\
\boldsymbol{u}\left(\boldsymbol{\omega}_{t}\right)=\mathbf{0}, & & \boldsymbol{\omega}_{t} \in \partial \Omega .
\end{array}\right.
$$

Since $\boldsymbol{\phi}(\boldsymbol{x}, t)$ is a diffeomorphic mapping with respect to $\boldsymbol{x}$, thus one has $\boldsymbol{x}=\boldsymbol{\phi}^{-1}\left(\boldsymbol{\omega}_{t}\right)$. Suppose also that $h\left(\boldsymbol{\omega}_{t}, t\right) \in \mathcal{H}_{g, \phi}(\Omega,[0,1])$ is the $\ell$-th order continuously differentiable on $\Omega$, from the above differential system, thus we can easily see that $\boldsymbol{u} \in \mathcal{C}^{\ell+1}\left(\Omega, \mathbb{R}^{2}\right)$. We furtherly restrict the uniform boundedness of the first derivatives of $\boldsymbol{u}$ in (4.4), so that such $\boldsymbol{u}$ has the characteristic of equicontinuity. In order to employ a regularization $\mathcal{S}[\boldsymbol{u}]$ of $\boldsymbol{u}$ in the proposed registration model, we modify the constraint set $\mathbf{D} \mathbf{C}_{h\left(\boldsymbol{\omega}_{t}\right)}(\Omega)$ as

$$
\mathbf{D C H}_{h_{t}}(\Omega):=\left\{\boldsymbol{u} \in \mathbf{D C}_{h\left(\boldsymbol{\omega}_{t}\right)}(\Omega) \cap H^{1}(\Omega)\right\}
$$

with the norm

$$
\|u\|_{\mathrm{DCH}}=\|u\|_{\mathcal{C}^{\ell+1}}+\|u\|_{H^{1}(\Omega)} .
$$

4.1. Compactness of the space $\mathbf{D C H}_{h_{t}}(\Omega)$. We expect that this new constraint set $\mathbf{D C H} h_{h_{t}}(\Omega)$ will have similar differential geometry properties to a diffeomorphic set $\mathbf{D C}_{h\left(\phi_{t}\right)}(\Omega)$, but the new set will be better in analysis for image registration problem, depending on the solution space for $u$.

Proving existence of solutions for a functional such as $\mathcal{E}(\boldsymbol{u})$ may proceed in different ways; below we establish the result by lower semi-continuity of the functional and sequential compactness of space.

Lemma 4.2 (The Bolzano-Weierstrass Theorem [9]). Assume that $\Omega$ is a compact closed set. Then every continuous function $\boldsymbol{u} \in \mathbf{D} \mathbf{C}_{h_{t}}(\Omega)$ on a compact set $\Omega$ is bounded, i.e., $\mathbf{D C}_{h_{t}}(\Omega)$ is a bounded function space in $\mathcal{C}^{\ell+1}$.

Definition 4.3. A subset $\mathcal{F}$ of $\mathcal{C}\left(\Omega, \mathbb{R}^{d}\right)$ is equicontinuous if for every $\varepsilon>0$ there exists some $\delta$ such that $\|\boldsymbol{\phi}(\boldsymbol{x})-\boldsymbol{\phi}(\boldsymbol{y})\|<\varepsilon$ for all $\boldsymbol{\phi} \in \mathcal{F}$, and $\|\boldsymbol{x}-\boldsymbol{y}\|<\delta, \boldsymbol{x}, \boldsymbol{y} \in \Omega$.

LEMma 4.4. For any $h\left(\boldsymbol{\omega}_{t}, t\right) \in \mathcal{H}_{g, \phi}(\Omega,[0,1])$, any bounded sequence $\mathcal{F}=\left\{\boldsymbol{u}^{i}\right\}_{i=1}^{+\infty} \subset \mathbf{D C}_{h_{t}}(\Omega)$ is equicontinuous in $\mathcal{C}^{\ell+1}$.

Proof. For any $\boldsymbol{u} \in \mathcal{F} \subset \mathbf{D C}_{h_{t}}(\Omega) \subset \mathbf{H B}^{\ell+1, \epsilon}$ and $\mu \in(0,1], \exists \mathcal{L}>0$, from (4.2) one has

$$
\left\{\begin{array}{cl}
\operatorname{div} \boldsymbol{u}(\boldsymbol{x})=-\frac{\partial h(\boldsymbol{x}, t)}{\partial t}, & \boldsymbol{x} \in \Omega_{\mathrm{in}} \\
\boldsymbol{u}(\boldsymbol{x})=\mathbf{0}, & \boldsymbol{x} \in \partial \Omega . \\
{[\boldsymbol{u}]_{\Omega}^{k, \epsilon}=\sup _{\boldsymbol{x}, \boldsymbol{y} \in \Omega, \boldsymbol{x} \neq \boldsymbol{y}} \frac{\left|\partial^{k} \boldsymbol{u}(\boldsymbol{x})-\partial^{k} \boldsymbol{u}(\boldsymbol{y})\right|}{|\boldsymbol{x}-\boldsymbol{y}|^{\epsilon}}<\mathcal{L},} & k=0,1, \ldots, \ell+1 .
\end{array}\right.
$$

Moreover, the bounded

$$
\Lambda^{k}:=\sup _{\boldsymbol{u} \in \mathcal{F}}[\boldsymbol{u}]_{\Omega}^{k, \epsilon}<\mathcal{L}, \quad \text { for } k=0,1, \ldots, \ell+1
$$


is defined. For any $\boldsymbol{x}, \boldsymbol{y} \in \Omega$, we have

$$
\|\boldsymbol{u}(\boldsymbol{y})-\boldsymbol{u}(\boldsymbol{x})\|_{\mathcal{C}^{\ell+1}} \leq(\ell+1) \mathcal{L}|\boldsymbol{y}-\boldsymbol{x}|^{\mu},
$$

if taking $\delta=\left(\frac{\varepsilon}{(\ell+1) \mathcal{L}}\right)^{1 / \epsilon}$, thus we deduce

$$
\|\boldsymbol{u}(\boldsymbol{y})-\boldsymbol{u}(\boldsymbol{x})\|_{\mathcal{C}^{\ell+1}} \leq \varepsilon
$$

which shows the assertion.

Combining the boundedness of $\mathbf{D} \mathbf{C}_{h_{t}}(\Omega)$ from Lemma 4.2 and the sequentially equicontinuity of $\mathbf{D C}_{h_{t}}(\Omega)$ in $\mathcal{C}^{\ell+1}$ from Lemma 4.4, we deduce that $\mathbf{D} \mathbf{C}_{h_{t}}(\Omega)$ is sequentially compact from Arzela-Ascoli Theorem. From the compactness of $H^{1}(\Omega)$ [52], it follows

LEMma 4.5. $\mathbf{D C H}_{h_{t}}(\Omega)$ is sequentially compact.

4.2. Optimal control relaxation for the diffeomorphic registration (4.1). In this part, we first consider the nonlinear dynamical system of the form

$$
\left\{\begin{aligned}
\frac{d}{d t} \boldsymbol{\omega}_{t} & =\frac{\boldsymbol{u}\left(\boldsymbol{\omega}_{t}\right)}{h\left(\boldsymbol{\omega}_{t}, t\right)} \\
\boldsymbol{\omega}(\boldsymbol{x}, 0) & =\boldsymbol{\omega}_{0}=\boldsymbol{x}
\end{aligned}\right.
$$

where $\boldsymbol{\omega}_{t}$ is the state function at time $t$ and $\boldsymbol{u}\left(\boldsymbol{\omega}_{t}\right)$ is control increment function, and $\boldsymbol{\omega}_{0}=$ $\boldsymbol{\omega}(\boldsymbol{x}, 0)=\boldsymbol{x}, \boldsymbol{\omega}_{1}=\boldsymbol{\omega}(\boldsymbol{x}, 1)=\boldsymbol{\varphi}(\boldsymbol{x})$ are the initial state and final state (solution) at $t=0$ and $t=1$, respectively. Moreover, $h\left(\boldsymbol{\omega}_{t}, t\right)$ is a real-valued continuously differentiable function with respect to $\boldsymbol{\omega}_{t}$ and time $t$.

To indirectly solve the diffeomorphic deformation $\varphi$ by using the construction, we will use $\mathbf{D C H}_{h_{t}}(\Omega)$ to replace the constraints $\operatorname{Diff}_{\varphi}^{f(\varphi)}(\Omega)$ in model (4.1), and propose a time-sequence registration model to find the optimal control increment $\boldsymbol{u}\left(\boldsymbol{\omega}_{t}\right)$ as follow

$$
\begin{gathered}
\min _{\boldsymbol{u}\left(\boldsymbol{\omega}_{t}\right) \in \mathbf{D} \mathbf{C H}_{h_{t}}(\Omega)}\left\{\mathcal{E}\left(\boldsymbol{\omega}_{t}, \boldsymbol{u}\right):=\mathcal{D}_{T, R}^{\alpha(t)}[\boldsymbol{u}]+\tau \mathcal{S}^{t}[\boldsymbol{u}]\right\} \\
\text { s.t. }\left\{\begin{aligned}
\frac{d}{d t} \boldsymbol{\omega}_{t}=\boldsymbol{v}\left(\boldsymbol{\omega}_{t}, t\right):=\frac{\boldsymbol{u}\left(\boldsymbol{\omega}_{t}\right)}{h\left(\boldsymbol{\omega}_{t}, t\right)}, \\
\boldsymbol{\omega}(\boldsymbol{x}, 0)=\boldsymbol{\omega}_{0}=\boldsymbol{x},
\end{aligned}\right.
\end{gathered}
$$

where $\mathcal{D}_{T, R}^{\alpha(t)}[\boldsymbol{u}]:=\mathcal{D}\left[R, T ; \boldsymbol{\omega}_{t}+\alpha(t) \boldsymbol{u}\left(\boldsymbol{\omega}_{t}\right)\right]=\frac{1}{2} \int_{\Omega}\left(T\left(\boldsymbol{\omega}_{t}+\alpha(t) \boldsymbol{u}\left(\boldsymbol{\omega}_{t}\right)\right)-R\left(\phi^{-1}\left(\boldsymbol{\omega}_{t}\right)\right)\right)^{2} d \boldsymbol{\omega}_{t}, \mathcal{S}^{t}[\boldsymbol{u}]:=$ $\frac{1}{2} \int_{\Omega}\left|\nabla \boldsymbol{u}\left(\boldsymbol{\omega}_{t}\right)\right|^{2} d \boldsymbol{\omega}_{t}, \tau>0$ and $\alpha(t)=\frac{\Delta t}{h\left(\boldsymbol{\omega}_{t}, t\right)}>0$. Using the temporal RK4 discretization in $[0,1]$, an update scheme of the diffeomorphic mapping $\boldsymbol{\omega}_{t_{k}}$ in (4.7) is given as

$$
\left\{\begin{aligned}
\boldsymbol{\omega}_{t_{k}}= & \mathbf{R K 4}\left(\boldsymbol{\omega}_{t_{k-1}}, \boldsymbol{v}, \Delta t\right), \\
\boldsymbol{u}\left(\boldsymbol{\omega}_{t_{k}}\right)= & \underset{\boldsymbol{u}\left(\boldsymbol{\omega}_{t_{k}}\right) \in \mathbf{D C H}_{t_{t_{k}}}(\Omega)}{\arg \min }\left\{\mathcal{E}^{t_{k}}(\boldsymbol{u}):=\mathcal{E}\left(\boldsymbol{\omega}_{t_{k}}, \boldsymbol{u}\right)\right\} \\
\boldsymbol{\omega}(\boldsymbol{x}, 0) & =\boldsymbol{\omega}_{0}=\boldsymbol{x}
\end{aligned}\right.
$$

The optimization energy $\mathcal{E}^{t_{k}}(\boldsymbol{u})$ in (4.8) is a non-convex functional, however the constraint $\boldsymbol{u}\left(\boldsymbol{\omega}_{t_{k}}\right) \in \mathbf{D C H}_{h_{t_{k}}}(\Omega)$ ensures that the deformation field $\boldsymbol{\omega}_{t_{k}}=\boldsymbol{\phi}\left(\boldsymbol{x}, t_{k}\right)$ is a diffeomorphic solution of (3.5), hence $\boldsymbol{\phi}\left(\boldsymbol{x}, t_{k}\right)$ is reversible and its inverse transform $\boldsymbol{x}=\boldsymbol{\phi}^{-1}\left(\boldsymbol{\omega}_{t_{k}}\right)$ is continuous. 
4.3. ALMM for the optimal control system. In the numerical implementation, we employ the ALMM algorithm [50] to solve time-sequence constrained optimization problem

$$
\min _{\boldsymbol{u}\left(\boldsymbol{\omega}_{\iota}\right) \in \mathbf{D} \mathbf{C} \mathbf{H}_{h_{\iota}}(\Omega)}\left\{\mathcal{E}^{\iota}(\boldsymbol{u}):=\mathcal{D}_{T, R}^{\alpha(\iota)}[\boldsymbol{u}]+\tau \mathcal{S}[\boldsymbol{u}]\right\} \quad \text { with } \iota:=t_{k},
$$

where $\boldsymbol{\omega}_{\iota}=\boldsymbol{\omega}_{t_{k}}$ denotes the state (coordinate system) at time $t_{k}$.

To proceed, we make the following assumptions:

Assumption 4.1. For any $\boldsymbol{x} \in \Omega$, there exists a constant $\mathcal{M}_{0}>0$, assume that two images $T(\boldsymbol{x})$ and $R(\boldsymbol{x})$ satisfies:

$$
\max \left\{\|T\|_{L^{\infty}(\Omega)},\|R\|_{L^{\infty}(\Omega)},\|\nabla T\|_{L^{\infty}(\Omega)},\left\|\nabla^{2} T\right\|_{L^{\infty}(\Omega)}\right\}<\mathcal{M}_{0}<+\infty ;
$$

hence $\|T-R\|_{L^{\infty}(\Omega)}<2 \mathcal{M}_{0}$. Moreover, assume that the regularization functional $\mathcal{S}[\boldsymbol{u}]$ in (4.9) statisfies that

(i) lower semi-continuity (LSC), i.e., let $\boldsymbol{u}_{j} \in \mathbf{D C H}_{h_{t}}(\Omega)$ and $\boldsymbol{u}_{j} \underset{\mathrm{L}^{1}(\Omega)}{*} \boldsymbol{u}$, then

$$
\mathcal{S}[\boldsymbol{u}] \leq \lim _{j \rightarrow+\infty} \mathcal{S}\left[\boldsymbol{u}_{j}\right]
$$

(ii) lower boundedness, i.e., there exists a constant $L, \forall \boldsymbol{u} \in \mathbf{D C H}_{h_{t}}(\Omega)$, one has

$$
\mathcal{S}[\boldsymbol{u}] \geq L
$$

Next, in addition to Assumption 4.1, let us analyze the properties of the energy functional $\mathcal{E}^{\iota}(\boldsymbol{u})$

Lemma 4.6 (Lower semi-continuity of $\mathcal{E}^{\iota}(\boldsymbol{u})$ ). Assume that $T(\boldsymbol{x})$ is differentiable with respect to $\boldsymbol{x}$. Then the functional $\mathcal{W}(\boldsymbol{u}):=\mathcal{D}_{T, R}^{\alpha(\iota)}[\boldsymbol{u}]$ is LSC and consequently $\mathcal{E}^{\iota}(\boldsymbol{u})$ from (4.9) is also $L S C$, i.e. for each $\epsilon>0$ and $\boldsymbol{u}\left(\boldsymbol{\omega}_{\iota}\right) \in \mathbf{D C H}_{h_{t}}(\Omega)$ with $|\mathcal{W}(\boldsymbol{u})|<\infty$, there exists a $\delta(\epsilon)>0$ such that for all $\boldsymbol{v} \in \mathbf{D C H}_{h_{t}}(\Omega)$ satisfying $\|\boldsymbol{u}-\boldsymbol{v}\|_{L^{2}(\Omega)}<\delta(\epsilon)$, the inequality holds: $\mathcal{W}(\boldsymbol{u})<\mathcal{W}(\boldsymbol{v})+\epsilon$.

Proof. Since the function $T(\boldsymbol{x})$ is differentiable, there exists a real number $\theta \in(0,1)$ such that

$$
T(\boldsymbol{x}+\boldsymbol{u})=T(\boldsymbol{x}+\boldsymbol{v})+\nabla T(\boldsymbol{s}) \cdot \boldsymbol{h}
$$

where $\boldsymbol{s}=\boldsymbol{x}+\theta \boldsymbol{u}+(1-\theta) \boldsymbol{v}$ and $\boldsymbol{h}=\boldsymbol{u}-\boldsymbol{v}$. Hence we have

$$
\mathcal{W}(\boldsymbol{u})=\mathcal{W}(\boldsymbol{v})+\int_{\Omega}\left((T(\boldsymbol{x}+\boldsymbol{v})-R(\boldsymbol{x}))(\nabla T(\boldsymbol{s}) \cdot \boldsymbol{h})+\boldsymbol{h}^{T}\left(\nabla T(\boldsymbol{s}) \nabla T(\boldsymbol{s})^{T}\right) \boldsymbol{h}\right) d \Omega,
$$

From Assumption 4.1, the above equation leads to

$$
|\mathcal{W}(\boldsymbol{u})-\mathcal{W}(\boldsymbol{v})| \leq c_{1}\|\boldsymbol{u}-\boldsymbol{v}\|_{L^{2}(\Omega)}+c_{2}\|\boldsymbol{u}-\boldsymbol{v}\|_{L^{2}(\Omega)}^{2}, c_{1} \geq 0, c_{2} \geq 0 .
$$

In this case, we have, if taking $\delta(\epsilon)=\left(-c_{1}+\sqrt{c_{1}^{2}+4 \epsilon c_{2}}\right) /\left(2 c_{2}\right), \mathcal{W}(\boldsymbol{u})<\mathcal{W}(\boldsymbol{v})+\epsilon$. Consequently combined with Assumption 4.1 for term (ii) i.e. LSC of $\mathcal{S}[\boldsymbol{u}]$, the functional $\mathcal{E}^{\iota}(\boldsymbol{u})$ from (4.9) is lower semi-continuous. This proves the Lemma.

TheOREM 4.7. The minimization problem (4.9) has at least one solution for any $\iota \in[0,1]$, $\alpha(\iota)$ and $\tau>0$.

Proof. The above result (Lemma 4.6), combined with the compactness of $\mathcal{E}^{\iota}(\boldsymbol{u})$ (Lemma 4.5) and boundedness of $\mathbf{D C H} h_{h_{\iota}}(\Omega)$ (i.e, $\boldsymbol{u}$ is bounded in $\mathbf{D} \mathbf{C H}_{h_{\iota}}(\Omega)$ ), completes the proof (see the general framework in [52, Prop. 38.12(d)]). 
To solve the control increment $\boldsymbol{u}\left(\boldsymbol{\omega}_{\iota}\right)$ of proposed optimal control problem (4.8), we first write (4.9) as a saddle point problem defined by

$$
\min _{\boldsymbol{u}_{\iota}} \max _{\lambda_{\iota}}\left\{\mathcal{L}^{\iota}(\boldsymbol{u}, \lambda):=\mathcal{E}^{\iota}(\boldsymbol{u})-\int_{\Omega} \lambda\left(\boldsymbol{\omega}_{\iota}\right)\left(\operatorname{div} \boldsymbol{u}\left(\boldsymbol{\omega}_{\iota}\right)+\left.\frac{\partial h\left(\boldsymbol{\omega}_{\iota}, t\right)}{\partial t}\right|_{t=\iota}\right) d \boldsymbol{\omega}_{\iota}\right\},
$$

where $\lambda_{\iota}=\lambda\left(\boldsymbol{\omega}_{\iota}\right)$ is a multiplier function. Obviously, the maximum w.r.t. $\lambda_{\iota}$ will be $+\infty$, unless $\operatorname{div} \boldsymbol{u}\left(\boldsymbol{\omega}_{\iota}\right)+\left.\frac{\partial h\left(\boldsymbol{\omega}_{\iota}, t\right)}{\partial t}\right|_{t=\iota}=0$, so this is equivalent to the original problem (4.9). But this equivalence is not very useful and computationally. To deal easily with it, we add two 'proximal point' terms

$$
\begin{aligned}
\min _{\boldsymbol{u}_{\iota}} \max _{\lambda_{\iota}}\left\{\mathcal{L}_{\beta}^{\iota}\left(\boldsymbol{u}, \lambda ; \boldsymbol{u}^{i}, \lambda^{i}\right):=\mathcal{L}^{\iota}(\boldsymbol{u}, \lambda)\right. & +\frac{1}{2 \gamma} \int\left\|\boldsymbol{u}\left(\boldsymbol{\omega}_{\iota}\right)-\boldsymbol{u}^{i}\left(\boldsymbol{\omega}_{\iota}\right)\right\|^{2} d \boldsymbol{\omega}_{\iota} \\
& \left.-\frac{1}{2 \beta} \int\left\|\lambda\left(\boldsymbol{\omega}_{\iota}\right)-\lambda^{i}\left(\boldsymbol{\omega}_{\iota}\right)\right\|^{2} d \boldsymbol{\omega}_{\iota}\right\},
\end{aligned}
$$

where $\gamma$ and $\beta>0$ are two penalty parameters. Maximization w.r.t. $\lambda$ is now trivial (a concave quadratic), yielding

$$
\lambda\left(\boldsymbol{\omega}_{\iota}\right)=\lambda^{i}\left(\boldsymbol{\omega}_{\iota}\right)-\beta\left(\operatorname{div} \boldsymbol{u}\left(\boldsymbol{\omega}_{\iota}\right)+\left.\frac{\partial h\left(\boldsymbol{\omega}_{\iota}, t\right)}{\partial t}\right|_{t=\iota}\right) .
$$

Inserting the above multiplier update $\lambda\left(\boldsymbol{\omega}_{\iota}\right)$ into (4.11) leads to

$$
\begin{aligned}
& \min _{\boldsymbol{u}}\left\{\mathcal{E}_{\boldsymbol{u}^{i}, \lambda^{i}}(\boldsymbol{u})\right.:=\mathcal{E}^{\iota}(\boldsymbol{u})-\int_{\Omega} \lambda^{i}\left(\boldsymbol{\omega}_{\iota}\right)\left(\operatorname{div} \boldsymbol{u}\left(\boldsymbol{\omega}_{\iota}\right)+\left.\frac{\partial h\left(\boldsymbol{\omega}_{\iota}, t\right)}{\partial t}\right|_{t=\iota}\right) d \boldsymbol{\omega}_{\iota} \\
&\left.+\frac{1}{2 \gamma} \int\left\|\boldsymbol{u}\left(\boldsymbol{\omega}_{\iota}\right)-\boldsymbol{u}^{i}\left(\boldsymbol{\omega}_{\iota}\right)\right\|^{2} d \boldsymbol{\omega}_{\iota}+\frac{\beta}{2} \int_{\Omega}\left(\operatorname{div} \boldsymbol{u}\left(\boldsymbol{\omega}_{\iota}\right)+\left.\frac{\partial h\left(\boldsymbol{\omega}_{\iota}, t\right)}{\partial t}\right|_{t=\iota}\right)^{2} d \boldsymbol{\omega}_{\iota}\right\} .
\end{aligned}
$$

Thus the iterative scheme of ALMM can be modified as

$$
\left\{\begin{array}{l}
\boldsymbol{u}^{i+1}\left(\boldsymbol{\omega}_{\iota}\right)=\arg \min _{\boldsymbol{u}} \mathcal{E}_{\boldsymbol{u}^{i}, \lambda^{i}}(\boldsymbol{u}) \\
\lambda^{i+1}\left(\boldsymbol{\omega}_{\iota}\right)=\lambda^{i}\left(\boldsymbol{\omega}_{\iota}\right)-\beta\left(\operatorname{div} \boldsymbol{u}^{i+1}\left(\boldsymbol{\omega}_{\iota}\right)+\left.\frac{\partial h\left(\boldsymbol{\omega}_{\iota}, t\right)}{\partial t}\right|_{t=\iota}\right) .
\end{array}\right.
$$

Hence a new iteration $\left(\boldsymbol{u}^{i+1}\left(\boldsymbol{\omega}_{\iota}\right), \lambda^{i+1}(\boldsymbol{x})\right)^{T}$ is solved.

4.4. Convergence analysis. We now study the convergence of the proposed algorithm based on (4.14). The next two Lemmas and Assumption can apply not just to the saddle point problem (4.10) but also to the alternation iterations (4.14).

Lemma 4.8. Let $\mathcal{X}$ be a closed convex set, $\mathcal{F}_{1}(\boldsymbol{v})$ and $\mathcal{F}_{2}(\boldsymbol{v})$ be convex functions on $\mathcal{X}$ and the first-order variation of $\mathcal{F}_{2}(\boldsymbol{v})$ exists. Assume that the solution set of the $\min \left\{\mathcal{F}_{1}(\boldsymbol{v})+\mathcal{F}_{2}(\boldsymbol{v}) \mid \boldsymbol{v} \in \mathcal{X}\right\}$ is nonempty. Then

$$
\boldsymbol{v}^{*}=\arg \min \left\{\mathcal{F}(\boldsymbol{v}):=\mathcal{F}_{1}(\boldsymbol{v})+\mathcal{F}_{2}(\boldsymbol{v}) \mid \boldsymbol{v} \in \mathcal{X}\right\}
$$

if and only if

$$
\mathcal{F}_{1}(\boldsymbol{v})-\mathcal{F}_{1}\left(\boldsymbol{v}^{*}\right)+\mathcal{F}_{2}^{\prime}\left(\boldsymbol{v}^{*}\right)\left(\boldsymbol{v}-\boldsymbol{v}^{*}\right) \geq 0, \exists \boldsymbol{v}^{*} \in \mathcal{X} \text { and } \forall \boldsymbol{v} \in \mathcal{X}
$$


Proof. (4.15) $\Longrightarrow(4.16)$ : since $\boldsymbol{v}^{*}$ is a minimizer of the convex functional $\mathcal{F}(\boldsymbol{v})$ on $\mathcal{X}$, then for any $\boldsymbol{v} \in \mathcal{X}$ and $\epsilon \in(0,1]$, we have

$$
\begin{gathered}
\mathcal{F}_{1}\left(\boldsymbol{v}^{*}+\epsilon\left(\boldsymbol{v}-\boldsymbol{v}^{*}\right)\right) \leq \epsilon \mathcal{F}_{1}(\boldsymbol{v})+(1-\epsilon) \mathcal{F}_{1}\left(\boldsymbol{v}^{*}\right), \\
\frac{\mathcal{F}_{1}\left(\boldsymbol{v}^{*}+\epsilon\left(\boldsymbol{v}-\boldsymbol{v}^{*}\right)\right)-\mathcal{F}_{1}\left(\boldsymbol{v}^{*}\right)}{\epsilon}+\frac{\mathcal{F}_{2}\left(\boldsymbol{v}^{*}+\epsilon\left(\boldsymbol{v}-\boldsymbol{v}^{*}\right)\right)-\mathcal{F}_{2}\left(\boldsymbol{v}^{*}\right)}{\epsilon} \geq 0,
\end{gathered}
$$

which yields

$$
\mathcal{F}_{1}(\boldsymbol{v})-\mathcal{F}_{1}\left(\boldsymbol{v}^{*}\right)+\frac{\mathcal{F}_{2}\left(\boldsymbol{v}^{*}+\epsilon\left(\boldsymbol{v}-\boldsymbol{v}^{*}\right)\right)-\mathcal{F}_{2}\left(\boldsymbol{v}^{*}\right)}{\epsilon} \geq 0 .
$$

Letting $\epsilon \rightarrow 0^{+}$, the above inequality can be written as

$$
\mathcal{F}_{1}(\boldsymbol{v})-\mathcal{F}_{1}\left(\boldsymbol{v}^{*}\right)+\mathcal{F}_{2}^{\prime}\left(\boldsymbol{v}^{*}\right)\left(\boldsymbol{v}-\boldsymbol{v}^{*}\right) \geq 0, \forall \boldsymbol{v} \in \mathcal{X} .
$$
then

(4.16) $\Longrightarrow(4.15)$ : since $\mathcal{F}_{2}(\boldsymbol{v})$ is convex, i.e., $\mathcal{F}_{2}\left(\boldsymbol{v}^{*}+\epsilon\left(\boldsymbol{v}-\boldsymbol{v}^{*}\right)\right) \leq \epsilon \mathcal{F}_{2}(\boldsymbol{v})+(1-\epsilon) \mathcal{F}_{2}\left(\boldsymbol{v}^{*}\right)$,

$$
\mathcal{F}_{2}(\boldsymbol{v})-\mathcal{F}_{2}\left(\boldsymbol{v}^{*}\right)-\frac{\mathcal{F}_{2}\left(\boldsymbol{v}^{*}+\epsilon\left(\boldsymbol{v}-\boldsymbol{v}^{*}\right)\right)-\mathcal{F}_{2}\left(\boldsymbol{v}^{*}\right)}{\epsilon} \geq 0 .
$$

Letting $\epsilon \rightarrow 0^{+}$, the above inequality can be written as

$$
\mathcal{F}_{2}(\boldsymbol{v})-\mathcal{F}_{2}\left(\boldsymbol{v}^{*}\right)-\mathcal{F}_{2}^{\prime}\left(\boldsymbol{v}^{*}\right)\left(\boldsymbol{v}-\boldsymbol{v}^{*}\right) \geq 0, \forall \boldsymbol{v} \in \mathcal{X} .
$$

By substituting it into (4.16), we obtain

$$
\mathcal{F}(\boldsymbol{v})-\mathcal{F}\left(\boldsymbol{v}^{*}\right)=\mathcal{F}_{1}(\boldsymbol{v})-\mathcal{F}_{1}\left(\boldsymbol{v}^{*}\right)+\mathcal{F}_{2}(\boldsymbol{v})-\mathcal{F}_{2}\left(\boldsymbol{v}^{*}\right) \geq 0, \forall \boldsymbol{v} \in \mathcal{X},
$$

which implies that $\boldsymbol{v}^{*}$ is a solution of $\min _{\boldsymbol{v} \in \mathcal{X}} \mathcal{F}(\boldsymbol{v})$. The proof is complete.

The above variational equivalence perspective is particularly valid for convex problems, where we can expect to solve the subproblems $\min _{\boldsymbol{u}} \mathcal{E}_{\boldsymbol{u}^{i}, \lambda^{i}}(\boldsymbol{u})$ in an alternatively iteration sense. This is reflected in the following assumption, which we require throughout this section.

Assumption 4.2. For any saddle point $\left(\boldsymbol{u}_{\iota}^{*}, \lambda_{\iota}^{*}\right)$ of problem (4.10), assume that there exists a positive constant $\delta_{\iota}$ and a closed box defined by

$$
\mathcal{N}\left(\left(\boldsymbol{u}_{\iota}^{*}, \lambda_{\iota}^{*}\right), \delta_{\iota}\right)=\left\{(\boldsymbol{u}, \lambda) \mid\left\|\boldsymbol{u}-\boldsymbol{u}^{*}\right\|_{L^{2}(\Omega)}^{2} \leq \delta_{\iota},\left\|\lambda-\lambda^{*}\right\|_{L^{2}(\Omega)}^{2} \leq \delta_{\iota}\right\}
$$

such that

$$
\begin{aligned}
\mathcal{E}^{\iota \prime}\left(\boldsymbol{u}_{\iota}\right) \boldsymbol{\xi} \boldsymbol{\xi}=\alpha^{2}(\iota) \int_{\Omega}\left(\left(T\left(\boldsymbol{\omega}_{t}+\boldsymbol{u}_{\iota}\right)-\right.\right. & \left.R\left(\phi^{-1}\left(\boldsymbol{\omega}_{t}\right)\right)\right)\left(\boldsymbol{\xi}^{T} \nabla^{2} T\left(\boldsymbol{\omega}_{t}+\boldsymbol{u}_{\iota}\right) \boldsymbol{\xi}\right) \\
& \left.+\boldsymbol{\xi}^{T}\left(\nabla T\left(\boldsymbol{\omega}_{t}+\boldsymbol{u}_{\iota}\right) \nabla T\left(\boldsymbol{\omega}_{t}+\boldsymbol{u}_{\iota}\right)^{T}\right) \boldsymbol{\xi}\right) d \boldsymbol{\omega}_{\iota} \geq 0
\end{aligned}
$$

for any $\left(\boldsymbol{u}_{\iota}, \lambda_{\iota}\right) \in \mathcal{N}\left(\left(\boldsymbol{u}_{\iota}^{*}, \lambda_{\iota}^{*}\right), \delta_{\iota}\right)$ and $\mathbf{0} \neq \boldsymbol{\xi} \in \mathbf{D C H}_{h_{t}}(\Omega)$.

One of the most important consequences of the above Assumption condition is that the $\mathcal{E}^{\iota}\left(\boldsymbol{u}_{\iota}\right)$ is a convex functional on $\mathcal{N}\left(\left(\boldsymbol{u}_{\iota}^{*}, \lambda_{\iota}^{*}\right), \delta_{\iota}\right)$. Now, we are ready to reformulate the saddle-point models (4.10) as special cases of mixed variational inequality for establishing the convergence for the scheme (4.14). 
Lemma 4.9. Let $\mathcal{E}^{\iota}\left(\boldsymbol{u}_{\iota}\right)$ satisfy Assumption 4.2 and $\left(\boldsymbol{u}_{\iota}^{*}, \lambda_{\iota}^{*}\right)$ be the saddle point of problem (4.10), i.e.,

$$
\mathcal{L}^{\iota}\left(\boldsymbol{u}_{\iota}^{*}, \lambda_{\iota}\right) \leq \mathcal{L}^{\iota}\left(\boldsymbol{u}_{\iota}^{*}, \lambda_{\iota}^{*}\right) \leq \mathcal{L}^{\iota}\left(\boldsymbol{u}_{\iota}, \lambda_{\iota}^{*}\right), \quad \forall\left(\boldsymbol{u}_{\iota}, \lambda_{\iota}\right) \in \mathcal{N}\left(\left(\boldsymbol{u}_{\iota}^{*}, \lambda_{\iota}^{*}\right), \delta_{\iota}\right),
$$

if and only if

$$
\left\{\begin{array}{c}
\mathcal{E}^{\iota}\left(\boldsymbol{u}_{\iota}\right)-\mathcal{E}^{\iota}\left(\boldsymbol{u}_{\iota}^{*}\right)+\int_{\Omega}\left(\boldsymbol{u}_{\iota}-\boldsymbol{u}_{\iota}^{*}\right) \nabla\left(\lambda_{\iota}^{*}\right) d \boldsymbol{\omega}_{\iota} \geq 0, \\
\int_{\Omega}\left(\lambda_{\iota}-\lambda_{\iota}^{*}\right)\left(\boldsymbol{d i v} \boldsymbol{u}_{\iota}^{*}+\left.\frac{\partial h\left(\boldsymbol{\omega}_{\iota}, t\right)}{\partial t}\right|_{t=\iota}\right) d \boldsymbol{\omega}_{\iota} \geq 0,
\end{array} \quad \forall\left(\boldsymbol{u}_{\iota}, \lambda_{\iota}\right) \in \mathcal{N}\left(\left(\boldsymbol{u}_{\iota}^{*}, \lambda_{\iota}^{*}\right), \delta_{\iota}\right) .\right.
$$

Especially, by substituting, we obtain

$$
\mathcal{E}^{\iota}\left(\boldsymbol{u}_{\iota}\right)-\mathcal{E}^{\iota}\left(\boldsymbol{u}_{\iota}^{*}\right)+\int_{\Omega}\left(\boldsymbol{u}_{\iota}-\boldsymbol{u}_{\iota}^{*}\right) \nabla\left(\lambda_{\iota}^{*}\right)+\left(\lambda_{\iota}-\lambda_{\iota}^{*}\right)\left(\boldsymbol{d i v} \boldsymbol{u}_{\iota}^{*}+\left.\frac{\partial h\left(\boldsymbol{\omega}_{\iota}, t\right)}{\partial t}\right|_{t=\iota}\right) d \boldsymbol{\omega}_{\iota} \geq 0
$$

for any $\left(\boldsymbol{u}_{\iota}, \lambda_{\iota}\right) \in \mathcal{N}\left(\left(\boldsymbol{u}_{\iota}^{*}, \lambda_{\iota}^{*}\right), \delta_{\iota}\right)$.

The mixed variational inequality serves as an unified mathematical model was presented in [22], for discrete convex optimization, we generalize it to infinite-dimensional saddle-point problem. From this point of view, it is natural to expect that the sequence (4.14) based on ALMM algorithm is convergent, provided $\left\{\left(\boldsymbol{u}_{\iota}^{k}, \lambda_{\iota}^{k}\right)\right\}$ satisfies the Fejér monotone condition [3]. We are now ready to show this important result which can be seen as a infinite-dimensional variant of [22].

Theorem 4.10. For any $\left(\boldsymbol{u}_{\iota}^{0}, \lambda_{\iota}^{0}\right) \in \mathcal{N}\left(\left(\boldsymbol{u}_{\iota}^{*}, \lambda_{\iota}^{*}\right), \delta_{\iota}\right)$, let $\mathcal{E}^{\iota}\left(\boldsymbol{u}_{\iota}\right)$ satisfy Assumption 4.2 and $\left(\boldsymbol{u}_{\iota}^{i+1}, \lambda_{\iota}^{i+1}\right)$ be the solution of the ALMM algorithm (4.14), and there exists a saddle point $\left(\overline{\boldsymbol{u}_{\iota}^{*}}, \overline{\lambda_{\iota}^{*}}\right)$ of problem (4.10) such that

$$
\boldsymbol{u}_{\iota}^{i} \stackrel{L^{2}(\Omega)}{\longrightarrow} \overline{\boldsymbol{u}_{\iota}^{*}}, \quad \lambda_{\iota}^{i} \stackrel{L^{2}(\Omega)}{\longrightarrow} \overline{\lambda_{\iota}^{*}}, \quad \text { as } i \rightarrow+\infty .
$$

Proof. Step 1. We should prove that $\left(\boldsymbol{u}_{\iota}^{i}, \lambda_{\iota}^{i}\right)$ is a convergent sequence on $\mathcal{N}\left(\left(\boldsymbol{u}_{\iota}^{*}, \lambda_{\iota}^{*}\right), \delta_{\iota}\right)$.

Since $\mathcal{E}^{\iota}(\boldsymbol{u})$ is a convex functional on $\mathcal{N}\left(\left(\boldsymbol{u}_{\iota}^{*}, \lambda_{\iota}^{*}\right), \delta_{\iota}\right)$, for any $\left(\boldsymbol{u}_{\iota}, \lambda_{\iota}\right) \in \mathcal{N}\left(\left(\boldsymbol{u}_{\iota}^{*}, \lambda_{\iota}^{*}\right), \delta_{\iota}\right)$, there exists the solution $\left(\boldsymbol{u}_{\iota}^{i+1}, \lambda_{\iota}^{i+1}\right) \in \mathcal{N}\left(\left(\boldsymbol{u}_{\iota}^{*}, \lambda_{\iota}^{*}\right), \delta_{\iota}\right)$ of the ALMM algorithm (4.14) satisfying that

$$
\left\{\begin{aligned}
\mathcal{E}^{\iota}\left(\boldsymbol{u}_{\iota}\right) & -\mathcal{E}^{\iota}\left(\boldsymbol{u}_{\iota}^{i+1}\right)+\frac{1}{\gamma} \int_{\Omega}\left(\boldsymbol{u}_{\iota}-\boldsymbol{u}_{\iota}^{i+1}\right)\left(\boldsymbol{u}_{\iota}^{i+1}-\boldsymbol{u}_{\iota}^{i}\right) d \boldsymbol{\omega}_{\iota} \\
& +\int_{\Omega}\left(\boldsymbol{u}_{\iota}-\boldsymbol{u}_{\iota}^{i+1}\right) \nabla\left(\lambda_{\iota}^{i}-\beta\left(\operatorname{div} \boldsymbol{u}_{\iota}^{i+1}+\left.\frac{\partial h\left(\boldsymbol{\omega}_{\iota}, t\right)}{\partial t}\right|_{t=\iota}\right)\right) d \boldsymbol{\omega}_{\iota} \geq 0 \\
& \int_{\Omega}\left(\lambda_{\iota}-\lambda_{\iota}^{i+1}\right)\left(\lambda_{\iota}^{i+1}-\lambda_{\iota}^{i}+\beta\left(\operatorname{div} \boldsymbol{u}_{\iota}^{i+1}+\left.\frac{\partial h\left(\boldsymbol{\omega}_{\iota}, t\right)}{\partial t}\right|_{t=\iota}\right)\right) d \boldsymbol{\omega}_{\iota} \geq 0
\end{aligned}\right.
$$

By taking the multiplier update $\lambda_{\iota}^{i+1}=\lambda_{\iota}^{i}-\beta\left(\operatorname{div} \boldsymbol{u}_{\iota}^{i+1}+\left.\frac{\partial h\left(\boldsymbol{\omega}_{\iota}, t\right)}{\partial t}\right|_{t=\iota}\right)$, we have

$$
\left\{\begin{aligned}
\mathcal{E}^{\iota}\left(\boldsymbol{u}_{\iota}\right) & -\mathcal{E}^{\iota}\left(\boldsymbol{u}_{\iota}^{i+1}\right)+\int_{\Omega}\left(\boldsymbol{u}_{\iota}-\boldsymbol{u}_{\iota}^{i+1}\right)\left(\nabla\left(\lambda_{\iota}^{i+1}\right)+\frac{1}{\gamma}\left(\boldsymbol{u}_{\iota}^{i+1}-\boldsymbol{u}_{\iota}^{i}\right)\right) d \boldsymbol{\omega}_{\iota} \geq 0, \\
& \int_{\Omega}\left(\lambda_{\iota}-\lambda_{\iota}^{i+1}\right)\left(\left(\operatorname{div} \boldsymbol{u}_{\iota}^{i+1}+\left.\frac{\partial h\left(\boldsymbol{\omega}_{\iota}, t\right)}{\partial t}\right|_{t=\iota}\right)+\frac{1}{\beta}\left(\lambda_{\iota}^{i+1}-\lambda_{\iota}^{i}\right)\right) d \boldsymbol{\omega}_{\iota} \geq 0 .
\end{aligned}\right.
$$

Let us recall that the Gauss formula and the divergence theorem can be employed to obtain

$$
\int_{\Omega}\left(\boldsymbol{u}_{\iota}-\boldsymbol{u}_{\iota}^{i+1}\right)\left(\nabla \lambda_{\iota}-\nabla \lambda_{\iota}^{i+1}\right)+\left(\operatorname{div} \boldsymbol{u}_{\iota}-\operatorname{div} \boldsymbol{u}_{\iota}^{i+1}\right)\left(\lambda_{\iota}-\lambda_{\iota}^{i+1}\right) d \boldsymbol{\omega}_{\iota}=0 .
$$


Combining (4.21) and (4.22), we derive

$$
\begin{aligned}
& \frac{1}{\gamma} \int_{\Omega}\left(\boldsymbol{u}_{\iota}-\boldsymbol{u}_{\iota}^{i+1}\right)\left(\boldsymbol{u}_{\iota}^{i+1}-\boldsymbol{u}_{\iota}^{i}\right) d \boldsymbol{\omega}_{\iota}+\frac{1}{\beta} \int_{\Omega}\left(\lambda_{\iota}-\lambda_{\iota}^{i+1}\right)\left(\lambda_{\iota}^{i+1}-\lambda_{\iota}^{i}\right) d \boldsymbol{\omega}_{\iota} \\
& \quad \geq \mathcal{E}^{\iota}\left(\boldsymbol{u}_{\iota}^{i+1}\right)-\mathcal{E}^{\iota}\left(\boldsymbol{u}_{\iota}\right)+\int_{\Omega}\left(\boldsymbol{u}_{\iota}^{i+1}-\boldsymbol{u}_{\iota}\right) \nabla\left(\lambda_{\iota}\right)+\left(\lambda_{\iota}^{i+1}-\lambda_{\iota}\right)\left(\operatorname{div} \boldsymbol{u}_{\iota}+\left.\frac{\partial h\left(\boldsymbol{\omega}_{\iota}, t\right)}{\partial t}\right|_{t=\iota}\right) d \boldsymbol{\omega}_{\iota} .
\end{aligned}
$$

If taking $\left(\boldsymbol{u}_{\iota}, \lambda_{\iota}\right)=\left(\boldsymbol{u}_{\iota}^{*}, \lambda_{\iota}^{*}\right)$ and following Lemma 4.9, the inequality (4.23) can be rewritten as

$$
\begin{aligned}
& \frac{1}{\gamma} \int_{\Omega}\left(\boldsymbol{u}_{\iota}^{*}-\boldsymbol{u}_{\iota}^{i+1}\right)\left(\boldsymbol{u}_{\iota}^{i+1}-\boldsymbol{u}_{\iota}^{i}\right) d \boldsymbol{\omega}_{\iota}+\frac{1}{\beta} \int_{\Omega}\left(\lambda_{\iota}^{*}-\lambda_{\iota}^{i+1}\right)\left(\lambda_{\iota}^{i+1}-\lambda_{\iota}^{i}\right) d \boldsymbol{\omega}_{\iota} \\
& \quad \geq \mathcal{E}^{\iota}\left(\boldsymbol{u}_{\iota}^{i+1}\right)-\mathcal{E}^{\iota}\left(\boldsymbol{u}_{\iota}^{*}\right)+\int_{\Omega}\left(\boldsymbol{u}_{\iota}^{i+1}-\boldsymbol{u}_{\iota}^{*}\right) \nabla\left(\lambda_{\iota}^{*}\right)+\left(\lambda_{\iota}^{i+1}-\lambda_{\iota}^{*}\right)\left(\operatorname{div} \boldsymbol{u}_{\iota}^{*}+\left.\frac{\partial h\left(\boldsymbol{\omega}_{\iota}, t\right)}{\partial t}\right|_{t=\iota}\right) d \boldsymbol{\omega}_{\iota} \geq 0 .
\end{aligned}
$$

Note that if $\int_{\Omega} \boldsymbol{f}^{T}(\boldsymbol{g}-\boldsymbol{f}) d \boldsymbol{\omega}_{\iota} \geq 0$, then

$$
\|\boldsymbol{f}\|_{L^{2}(\Omega)}^{2}=\int_{\Omega} \boldsymbol{f}^{T} \boldsymbol{f} d \boldsymbol{\omega}_{\iota} \leq \int_{\Omega} \boldsymbol{g}^{T} \boldsymbol{g} d \boldsymbol{\omega}_{\iota}-\int_{\Omega}(\boldsymbol{g}-\boldsymbol{f})^{T}(\boldsymbol{g}-\boldsymbol{f}) d \boldsymbol{\omega}_{\iota}=\|\boldsymbol{g}\|_{L^{2}(\Omega)}^{2}-\|\boldsymbol{g}-\boldsymbol{f}\|_{L^{2}(\Omega)}^{2} .
$$

The fact that the left side in (4.24) satisfies $\int_{\Omega} \boldsymbol{f}^{T}(\boldsymbol{g}-\boldsymbol{f}) d \boldsymbol{\omega}_{\iota} \geq 0$ implies that

$$
\varpi^{i+1}+\vartheta^{i+1} \leq \varpi^{i}
$$

where $\varpi^{i+1}:=\frac{\beta}{\gamma}\left\|\boldsymbol{u}_{\iota}^{i+1}-\boldsymbol{u}_{\iota}^{*}\right\|_{L^{2}(\Omega)}^{2}+\left\|\lambda_{\iota}^{i+1}-\lambda_{\iota}^{*}\right\|_{L^{2}(\Omega)}^{2}$ and $\vartheta^{i+1}:=\frac{\beta}{\gamma}\left\|\boldsymbol{u}_{\iota}^{i+1}-\boldsymbol{u}_{\iota}^{i}\right\|_{L^{2}(\Omega)}^{2}+\| \lambda_{\iota}^{i+1}-$ $\lambda_{\iota}^{i} \|_{L^{2}(\Omega)}^{2}$. Since the sequence $\left\{\varpi^{i+1}\right\}$ is positive, bounded and nonincreasing, thus it is convergent and so $\left\{\vartheta^{i+1}\right\}$ tends to zero, hence there exists $\left(\overline{\boldsymbol{u}_{\iota}^{*}}, \overline{\lambda_{\iota}^{*}}\right) \in \mathcal{N}\left(\left(\boldsymbol{u}_{\iota}^{*}, \lambda_{\iota}^{*}\right), \delta_{\iota}\right)$, we deduce from the preceding inequalities that

$$
\left(\boldsymbol{u}_{\iota}^{i}, \lambda_{\iota}^{i}\right) \stackrel{L^{2}(\Omega)}{\longrightarrow}\left(\overline{\boldsymbol{u}_{\iota}^{*}}, \overline{\lambda_{\iota}^{*}}\right) \quad \text { as } i \rightarrow+\infty,
$$

where the sequence $\left\{\left(\boldsymbol{u}_{\iota}^{i}, \lambda_{\iota}^{i}\right)\right\}$ generated by (4.14) is Fejér monotone sequence [3].

Step 2. Next, we have to prove that $\left(\overline{\boldsymbol{u}_{\iota}^{*}}, \overline{\lambda_{\iota}^{*}}\right)$ is a saddle point of the Lagrangian functional $\mathcal{L}^{\iota}(\boldsymbol{u}, \lambda)$. For any $\left(\boldsymbol{u}_{\iota}, \lambda_{\iota}\right) \in \mathcal{N}\left(\left(\boldsymbol{u}_{\iota}^{*}, \lambda_{\iota}^{*}\right), \delta_{\iota}\right)$, let us consider

$$
\left\{\begin{aligned}
\lim _{i \rightarrow+\infty}\left\{\mathcal{E}^{\iota}\left(\boldsymbol{u}_{\iota}\right)\right. & \left.-\mathcal{E}^{\iota}\left(\boldsymbol{u}_{\iota}^{i+1}\right)+\int_{\Omega}\left(\boldsymbol{u}_{\iota}-\boldsymbol{u}_{\iota}^{i+1}\right)\left(\nabla\left(\lambda_{\iota}^{i+1}\right)+\frac{1}{\gamma}\left(\boldsymbol{u}_{\iota}^{i+1}-\boldsymbol{u}_{\iota}^{i}\right)\right) d \boldsymbol{\omega}_{\iota}\right\} \\
& =\mathcal{E}^{\iota}\left(\boldsymbol{u}_{\iota}\right)-\mathcal{E}^{\iota}\left(\overline{\boldsymbol{u}_{\iota}^{*}}\right)+\int_{\Omega}\left(\boldsymbol{u}_{\iota}-\overline{\boldsymbol{u}_{\iota}^{*}}\right) \nabla\left(\overline{\lambda_{\iota}^{*}}\right) d \boldsymbol{\omega}_{\iota} \geq 0, \\
\lim _{i \rightarrow+\infty} & \int_{\Omega}\left(\lambda_{\iota}-\lambda_{\iota}^{i+1}\right)\left(\left(\operatorname{div} \boldsymbol{u}_{\iota}^{i+1}+\left.\frac{\partial h\left(\boldsymbol{\omega}_{\iota}, t\right)}{\partial t}\right|_{t=\iota}\right)+\frac{1}{\beta}\left(\lambda_{\iota}^{i+1}-\lambda_{\iota}^{i}\right)\right) d \boldsymbol{\omega}_{\iota} \\
& =\int_{\Omega}\left(\lambda_{\iota}-\overline{\lambda_{\iota}^{*}}\right)\left(\operatorname{div} \overline{\boldsymbol{u}_{\iota}^{*}}+\left.\frac{\partial h\left(\boldsymbol{\omega}_{\iota}, t\right)}{\partial t}\right|_{t=\iota}\right) d \boldsymbol{\omega}_{\iota} \geq 0 .
\end{aligned}\right.
$$

which implies from Lemma 4.9 that $\left(\overline{\boldsymbol{u}_{\iota}^{*}}, \overline{\lambda_{\iota}^{*}}\right)$ is the saddle point of the Lagrangian functional $\mathcal{L}^{\iota}(\boldsymbol{u}, \lambda)$.

REMARK 4. From Theorem 4.10 and the constraint optimization theory [21], let $\mathcal{E}^{\iota}\left(\boldsymbol{u}_{\iota}\right)$ satisfy Assumption 4.2, then we may observe the following:

1. $\overline{\boldsymbol{u}}_{\iota}{ }^{*}:=\overline{\boldsymbol{u}^{*}\left(\boldsymbol{\omega}_{\iota}\right)}$ is the solution of the constraint optimization (4.9) with $\iota=t_{k}$;

2. $\left\{\boldsymbol{\omega}_{t_{k}}:=\phi\left(\boldsymbol{x}, t_{k}\right)\right\}$ is a convergent sequence and converges to the solution $\boldsymbol{\varphi}^{*}:=\boldsymbol{\phi}^{*}(\boldsymbol{x}, 1)$ of the diffeomorphic image registration model (4.1) as $t_{k} \rightarrow 1(k \rightarrow+\infty)$. 
4.5. Numerical implementation. It can be seen from formula (4.14) that the main work of seeking the optimal solution of the model is to solve the subproblem

$$
\min _{\boldsymbol{u}\left(\boldsymbol{\omega}_{\iota}\right)} \mathcal{E}_{\boldsymbol{u}^{i}, \lambda^{i}}(\boldsymbol{u})
$$

so the following discussion will focus on minimizing $\mathcal{E}_{\boldsymbol{u}^{i}, \lambda^{i}}(\boldsymbol{u})$. To obtain the optimal solution of (4.27), the first-order variation of $\mathcal{E}_{\boldsymbol{u}^{i}, \lambda^{i}}(\boldsymbol{u})$ can be used to deduce the Euler-Lagrange equation as follow (see [16] for more details)

$$
\begin{aligned}
\alpha(\iota) & {\left[T\left(\boldsymbol{\omega}_{\iota}+\alpha(\iota) \boldsymbol{u}\left(\boldsymbol{\omega}_{\iota}\right)\right)-R\left(\phi^{-1}\left(\boldsymbol{\omega}_{\iota}\right)\right)\right] \nabla T\left(\boldsymbol{\omega}_{\iota}+\alpha(\iota) \boldsymbol{u}\left(\boldsymbol{\omega}_{\iota}\right)\right)-\tau \Delta \boldsymbol{u}\left(\boldsymbol{\omega}_{\iota}\right) } \\
& +\beta\left(\nabla\left(-\left.\frac{\partial h\left(\boldsymbol{\omega}_{\iota}, t\right)}{\partial t}\right|_{t=\iota}+\frac{\lambda^{i}\left(\boldsymbol{\omega}_{\iota}\right)}{\beta}\right)-\nabla \operatorname{div} \boldsymbol{u}\left(\boldsymbol{\omega}_{\iota}\right)\right)+\frac{1}{\gamma}\left(\boldsymbol{u}\left(\boldsymbol{\omega}_{\iota}\right)-\boldsymbol{u}^{i}\left(\boldsymbol{\omega}_{\iota}\right)\right)=0
\end{aligned}
$$

with the boundary condition $\frac{\partial \boldsymbol{u}}{\partial \boldsymbol{n}}=\mathbf{0}$ on $\partial \Omega$. Finally, combining (4.28) and the boundary condition in (4.3), the solving nonlinear systems can be rewritten as

$$
\left\{\begin{array}{rlrl}
-\Delta \boldsymbol{u}\left(\boldsymbol{\omega}_{\iota}\right)-\frac{\beta}{\tau} \nabla \operatorname{div} \boldsymbol{u}\left(\boldsymbol{\omega}_{\iota}\right)+\frac{1}{\gamma} \boldsymbol{u}\left(\boldsymbol{\omega}_{\iota}\right)= & \boldsymbol{r}\left(\boldsymbol{\omega}_{\iota}+\alpha(\iota) \boldsymbol{u}\left(\boldsymbol{\omega}_{\iota}\right)\right), & & \text { in } \Omega_{\text {in }} \\
\boldsymbol{u}\left(\boldsymbol{\omega}_{\iota}\right)=\mathbf{0} \text { and } \frac{\partial \boldsymbol{u}\left(\boldsymbol{\omega}_{\iota}\right)}{\partial \boldsymbol{n}}=\mathbf{0}, & & \text { on } \partial \Omega,
\end{array}\right.
$$

where

$$
\begin{aligned}
\boldsymbol{r}\left(\boldsymbol{\omega}_{\iota}+\alpha(\iota) \boldsymbol{u}\left(\boldsymbol{\omega}_{\iota}\right)\right):=-\frac{\alpha(\iota)}{\tau}\left[T\left(\boldsymbol{\omega}_{\iota}+\alpha(\iota) \boldsymbol{u}\left(\boldsymbol{\omega}_{\iota}\right)\right)-R\left(\phi^{-1}\left(\boldsymbol{\omega}_{\iota}\right)\right)\right] \times \\
\nabla T\left(\boldsymbol{\omega}_{\iota}+\alpha(\iota) \boldsymbol{u}\left(\boldsymbol{\omega}_{\iota}\right)\right)-\frac{\beta}{\tau} \nabla\left(-\left.\frac{\partial h\left(\boldsymbol{\omega}_{\iota}, t\right)}{\partial t}\right|_{t=\iota}+\frac{\lambda^{i}\left(\boldsymbol{\omega}_{\iota}\right)}{\beta}\right)+\frac{1}{\gamma} \boldsymbol{u}^{i}\left(\boldsymbol{\omega}_{\iota}\right) .
\end{aligned}
$$

Therefore, the minimum point $\boldsymbol{u}\left(\boldsymbol{\omega}_{\iota}\right)$ of $\mathcal{E}_{\boldsymbol{u}^{i}, \lambda^{i}}(\boldsymbol{u})$ can be transformed into the solution of the nonlinear differential equations (4.29).

4.5.1. Discrete setting. Before we present some numerical results from applying the diffemorphic registration model, let us first introduce the discrete setting which we will use in the rest of this paper. Without loss of generality, we restrict our attention to $2 \mathrm{D}$ in the following part.

To find the finite difference scheme associated with the equation (4.29), we establish a uniform Cartesian mesh of size $m \times n$ with cell centers: $\left\{\left(x_{i}, y_{j}\right):=\left(i h_{x}, j h_{y}\right): i=1, \ldots, m, j=1, \ldots, n\right\}$, where $h_{x}=1 / m$ and $h_{y}=1 / n$ denote the spacing step length and $(i, j)$ denotes the index of the locations $\left(i h_{x}, j h_{y}\right)$ in image domain $\Omega:=[0,1]^{2}$. The grid line intersections (or grid nodes) are labeled using fractional indices. For example, the grid node in the upper left corner of the cell centered at $(i, j)$ is labeled $\left(i-\frac{1}{2}, j-\frac{1}{2}\right)$. Fig.2 illustrates the labeling scheme. Here for the time step $\iota=t_{k} \in[0,1]$, the boundary conditions $\left.\boldsymbol{u}\left(\boldsymbol{\omega}_{\iota}\right)\right|_{\partial \Omega}=\mathbf{0}$ and $\frac{\partial \boldsymbol{u}}{\partial \boldsymbol{n}}=\mathbf{0}$ of the control increment $\boldsymbol{u}$ are added, i.e.,

$$
\begin{array}{r}
u_{\frac{1}{2}, j}^{l}=0, \quad u_{m+\frac{1}{2}, j}^{l}=0, \quad u_{i, \frac{1}{2}}^{l}=0, \quad u_{i, n+\frac{1}{2}}^{l}=0, \\
u_{0, j}^{l}=u_{1, j}^{l}, \quad u_{m, j}^{l}=u_{m+1, j}^{l}, \quad u_{i, 0}^{l}=u_{i, 1}^{l}, \quad u_{i, n}^{l}=u_{i, n+1}^{l}, \\
\text { for all } i=1, \ldots, m, \quad j=1, \ldots, n \text { and } l=1,2 .
\end{array}
$$

Naturally, the simplest method is to consider separately the discretization of each second-order derivative in $x$ and $y$, which is equivalent to using the one-dimensional approximation. By doing 
so, let us define the discrete schemes applied to a scalar function $v$ and a vectorial $\boldsymbol{u}=\left(u^{1}, u^{2}\right)$ at the grid point $(i, j)$ by

$$
\begin{gathered}
(\nabla v)_{i j}=\left(\delta_{x} v_{i j}, \delta_{y} v_{i j}\right), \quad(\operatorname{div} \boldsymbol{u})_{i j}=\delta_{x} u_{i j}^{1}+\delta_{y} u_{i j}^{2}, \\
\left(\Delta u^{l}\right)_{i j}=\delta_{x x} u_{i j}^{l}+\delta_{y y} u_{i j}^{l}, \quad(\Delta \boldsymbol{u})_{i j}=\left(\left(\Delta u^{1}\right)_{i j},\left(\Delta u^{2}\right)_{i j}\right),
\end{gathered}
$$

where

$$
\begin{gathered}
\delta_{x} v_{i, j}=\left(v_{i+1, j}-v_{i-1, j}\right) /\left(2 h_{x}\right), \delta_{y} v_{i, j}=\left(v_{i, j+1}-v_{i, j-1}\right) /\left(2 h_{y}\right), \\
\delta_{x x} u_{i, j}^{l}=\left(u_{i-1, j}^{l}-2 u_{i, j}^{l}+u_{i+1, j}^{l}\right) / h_{x}^{2}, \delta_{y y} u_{i, j}^{l}=\left(u_{i, j-1}^{l}-2 u_{i, j}^{l}+u_{i, j+1}^{l}\right) / h_{y}^{2} .
\end{gathered}
$$

As mentioned previously, the gradient $\nabla T$ is obtained from the first variation of $\operatorname{SSD}(\boldsymbol{u})$. To discrete $\nabla T$ at cell centers around boundary $\partial \Omega$, it would be interesting to take into account the Neumann boundary condition $\frac{\partial T(\boldsymbol{x})}{\partial \boldsymbol{n}}=0$ on $\partial \Omega$, i.e

$$
\begin{aligned}
T_{0, j}=T_{1, j}, \quad T_{m+1, j}=T_{m, j}, \quad T_{i, 0}=T_{i, 1}, \quad T_{i, n+1}=T_{i, n}, \\
\\
\text { for all } i=1, \ldots, m, \quad j=1, \ldots, n .
\end{aligned}
$$

Then we have

$$
\begin{gathered}
(\nabla T)_{i j}=\left(\delta_{x} T_{i j}, \delta_{y} T_{i j}\right), \\
\delta_{x} T_{i j}=\left(T_{i+1, j}-T_{i-1, j}\right) /\left(2 h_{x}\right) ; \quad \delta_{y} T_{i j}=\left(T_{i, j+1}-T_{i, j-1}\right) /\left(2 h_{y}\right) .
\end{gathered}
$$

Here we employ bicubic interpolator, which takes more surrounding pixels into consideration, to obtain the most image information at the non-grid point from the weighted average of the most recent 16 sampling points in the rectangular grid.

To summarize, the approximation of (4.29) is then given by

$$
\begin{aligned}
-\left(\Delta u^{1}\right)_{i j}-\frac{\beta}{\tau} \delta_{x}\left((\operatorname{div} \boldsymbol{u})_{i j}\right)+\frac{1}{\gamma} u_{i j}^{1}= & -\frac{\alpha\left(t_{k}\right)}{\tau}\left(T^{k}-R\right)_{i j} \delta_{x}\left(\left(T^{k}\right)_{i j}\right) \\
& +\frac{\beta}{\tau} \delta_{x}\left(\left(h^{\prime}\left(\cdot, t_{k}\right)-\frac{\lambda(\cdot)}{\beta}\right)_{i j}\right)+\frac{1}{\gamma} \bar{u}_{i j}^{1}, \\
-\left(\Delta u^{2}\right)_{i j}-\frac{\beta}{\tau} \delta_{y}\left((\operatorname{div} \boldsymbol{u})_{i j}\right)+\frac{1}{\gamma} u_{i j}^{2}= & -\frac{\alpha\left(t_{k}\right)}{\tau}\left(T^{k}-R\right)_{i j} \delta_{y}\left(\left(T^{k}\right)_{i j}\right) \\
& +\frac{\beta}{\tau} \delta_{y}\left(\left(h^{\prime}\left(\cdot, t_{k}\right)-\frac{\lambda(\cdot)}{\beta}\right)_{i j}\right)+\frac{1}{\gamma} \bar{u}_{i j}^{2},
\end{aligned}
$$

where $T^{k}=T\left(\boldsymbol{\omega}_{t_{k}}+\alpha\left(t_{k}\right) \boldsymbol{u}\left(\boldsymbol{\omega}_{t_{k}}\right)\right)$ and $h^{\prime}\left(\cdot, t_{k}\right)=\left.\frac{\partial h\left(\boldsymbol{\omega}_{t_{k}}, t\right)}{\partial t}\right|_{t=t_{k}}$. Now we can consider the augmented Lagrangian multiplier update (4.12), which can be seen as the combination of the previous discretization. So the discrete scheme is

$$
\left(\lambda_{k}^{\ell+1}\right)_{i j}=\left(\lambda_{k}^{\ell}\right)_{i j}-\beta\left(\operatorname{div} \boldsymbol{u}^{\ell+1}+\left.\frac{\partial h\left(\boldsymbol{\omega}_{t_{k}}, t\right)}{\partial t}\right|_{t=t_{k}}\right)_{i j} .
$$

4.5.2. Deformation quality indicator and folding correction. As has been discussed, the folding of deformed mesh in diffeomorphic image registration, measured often by the local quantity $\operatorname{det}(\nabla \boldsymbol{\varphi}(\boldsymbol{x}))$, should be reduced or avoided. Especially in infinite-dimension case, $\operatorname{det}(\nabla \boldsymbol{\varphi}(\boldsymbol{x}))>0$ ensures that the deformation $\boldsymbol{\varphi}(\boldsymbol{x})$ is an one-to-one mapping.

Grid unfolding indicator. We are motivated by a finite volume approach of Haber and Modersitzki $[23,24]$ to discuss the Jacobian determinant value at the cell center $(i, j)$ in 2D. 


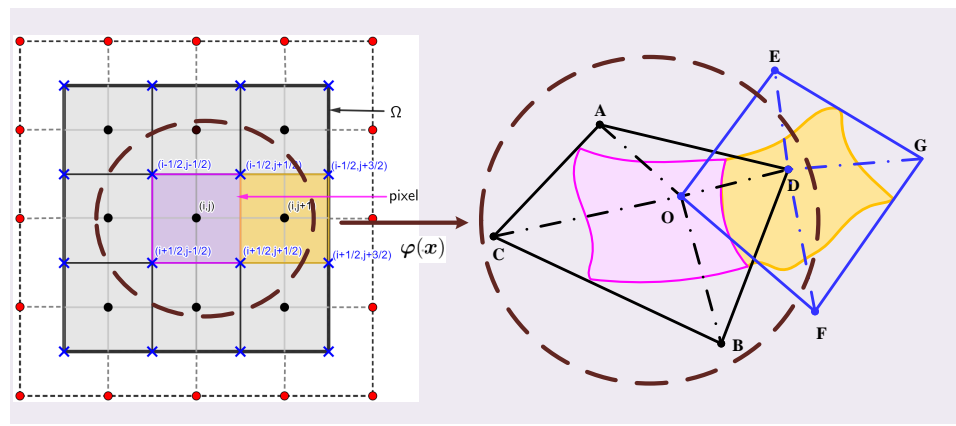

(a) 2D partition

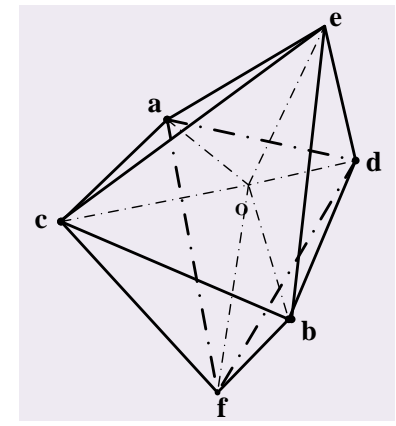

(b) 3D partition

FIG. 2. Cartesian grid, deformation $\boldsymbol{\varphi ( x )}$ and triangle partition for deformation quality evaluations in $2 D$ and $3 D$ case, and overlapping correction, where five adjacent cell centers in $2 D$ are denoted by $A:=\varphi_{i-1, j}=$ $\left(\varphi_{i-1, j}^{1}, \varphi_{i-1, j}^{2}\right), B:=\varphi_{i+1, j}=\left(\varphi_{i+1, j}^{1}, \varphi_{i+1, j}^{2}\right), C:=\varphi_{i, j-1}=\left(\varphi_{i, j-1}^{1}, \varphi_{i, j-1}^{2}\right), D:=\varphi_{i, j+1}=\left(\varphi_{i, j+1}^{1}, \varphi_{i, j+1}^{2}\right)$ and $O:=\varphi_{i j}=\left(\varphi_{i j}^{1}, \varphi_{i j}^{2}\right)$.

For a straightforward central-difference discretization, the unfolding quantity $\left.\operatorname{det}(\nabla \varphi)\right|_{O}$ of the deformation $O:=\varphi_{i j}$ at cell center $(i, j)$ is given by

$$
\begin{aligned}
&\left.\operatorname{det}(\nabla \varphi)\right|_{O}=\left|\begin{array}{cc}
\delta_{x} \varphi_{i j}^{1} & \delta_{y} \varphi_{i j}^{1} \\
\delta_{x} \varphi_{i j}^{2} & \delta_{y} \varphi_{i j}^{2}
\end{array}\right|=\frac{1}{4 h_{x} h_{y}}\left|\begin{array}{cc}
\varphi_{i+1, j}^{1}-\varphi_{i-1, j}^{1} & \varphi_{i, j+1}^{1}-\varphi_{i, j-1}^{1} \\
\varphi_{i+1, j}^{2}-\varphi_{i-1, j}^{2} & \varphi_{i, j+1}^{2}-\varphi_{i, j-1}^{2}
\end{array}\right| \\
&= \frac{1}{4 h_{x} h_{y}}\left(\left|\begin{array}{cc}
\varphi_{i+1, j}^{1}-\varphi_{i j}^{1} & \varphi_{i, j+1}^{1}-\varphi_{i j}^{1} \\
\varphi_{i+1, j}^{2}-\varphi_{i j}^{2} & \varphi_{i, j+1}^{2}-\varphi_{i j}^{2}
\end{array}\right|+\left|\begin{array}{cc}
\varphi_{i, j+1}^{1}-\varphi_{i j}^{1} & \varphi_{i-1, j}^{1}-\varphi_{i j}^{1} \\
\varphi_{i, j+1}^{2}-\varphi_{i j}^{2} & \varphi_{i-1, j}^{2}-\varphi_{i j}^{2}
\end{array}\right|\right. \\
&\left.\quad+\left|\begin{array}{ll}
\varphi_{i-1, j}^{1}-\varphi_{i j}^{1} & \varphi_{i, j-1}^{1}-\varphi_{i j}^{1} \\
\varphi_{i-1, j}^{2}-\varphi_{i j}^{2} & \varphi_{i, j-1}^{2}-\varphi_{i j}^{2}
\end{array}\right|+\left|\begin{array}{cc}
\varphi_{i, j-1}^{1}-\varphi_{i j}^{1} & \varphi_{i+1, j}^{1}-\varphi_{i j}^{1} \\
\varphi_{i, j-1}^{2}-\varphi_{i j}^{2} & \varphi_{i+1, j}^{2}-\varphi_{i j}^{2}
\end{array}\right|\right) \\
&=\frac{1}{2}\left(R_{\Delta O B D}^{i j}+R_{\Delta O D A}^{i j}+R_{\Delta O A C}^{i j}+R_{\Delta O C B}^{i j}\right),
\end{aligned}
$$

where five adjacent cell centers $\left(O, A, B, C\right.$ and $D$ in Fig.2(a)) are involved, and $R_{\triangle O B D}^{i j}$ is an area ratio of the triangle signed area $\frac{1}{2}\left|\begin{array}{cc}\varphi_{i+1, j}^{1}-\varphi_{i j}^{1} & \varphi_{i, j+1}^{1}-\varphi_{i j}^{1} \\ \varphi_{i+1, j}^{2}-\varphi_{i j}^{2} & \varphi_{i, j+1}^{2}-\varphi_{i j}^{2}\end{array}\right|$ to the area element $h_{x} h_{y}$. However, if we allow the deformation $\varphi$ to shrink or enlarge area in a certain amount, the deformation is allowed to be much more irregular and the displacement regularity is not guaranteed even if the above value $\left.\operatorname{det}(\nabla \varphi)\right|_{O}>0$. An intuitive example is illustrated in Fig.3(a), where the movement of the right point $D$ results in a "twist" of the box, but this twist can not be observed by computing the quantity $\left.\operatorname{det}(\nabla \varphi)\right|_{O}=\frac{1}{2}\left(\left|R_{\triangle O B D}^{i j}\right|-\left|R_{\triangle O D A}^{i j}\right|+\left|R_{\triangle O A C}^{i j}\right|+\left|R_{\triangle O C B}^{i j}\right|\right)$. Since the ratio $R_{\triangle O D A}^{i j}$ computed by the triangle $\triangle O D A$ is negative and each one of the other ratios is positive. Especially when point $D$ is close to $A$ in Fig.3(a), the quantity $\left.\operatorname{det}(\nabla \varphi)\right|_{O}$ may even be large although a twist has occurred.

To prevent twists and singular points, Haber and Modersitzki [24] demanded that the area of every deformed box is preserved for vertex grid discretization. However, a cell center grid (see the black points in Fig.2(a)) and the central-difference are used in this work, we therefore propose a signed area minimum ratio indicator that can detect twists of the grid, i.e.,

$$
R_{O}=R_{i j}:=\min \left(R_{\triangle O B D}^{i j}, R_{\triangle O D A}^{i j}, R_{\triangle O A C}^{i j}, R_{\triangle O C B}^{i j}\right) .
$$

Based on the previous considerations, a triangle cannot twist without its measure $R_{i j}$ to change 
sign, so we consider our grid quality evaluation on a triangulation. If a triangle folds, its $R_{i j}$ becomes negative. Such an evaluation is consistent even in cases of large deformations.

Similar to 2D case, we then also discuss the extension to 3D. The deformation $\varphi$ is discretized at the center of each voxel. Once again, a Jacobian determinant $\left.\operatorname{det}(\nabla \varphi)\right|_{o}$ of the deformation $o:=\varphi_{i j k}$ based on the deformed center $(i, j, k)$ cannot detect twists. Therefore, every octahedron with six corners $a, b, c, d, e, f$ and one center $o$ is divided into eight tetrahedrons; cf. Fig.2(b). The Jacobian determinant with respect to $(i, j, k)$ can be defined by

$$
\begin{aligned}
& \left.\operatorname{det}(\nabla \varphi)\right|_{o}=\left|\begin{array}{lll}
\delta_{x} \varphi_{i j k}^{1} & \delta_{y} \varphi_{i j k}^{1} & \delta_{z} \varphi_{i j k}^{1} \\
\delta_{x} \varphi_{i j k}^{2} & \delta_{y} \varphi_{i j k}^{2} & \delta_{z} \varphi_{i j k}^{2} \\
\delta_{x} \varphi_{i j k}^{3} & \delta_{y} \varphi_{i j k}^{3} & \delta_{z} \varphi_{i j k}^{3}
\end{array}\right| \\
& =\frac{1}{8 h_{x} h_{y} h_{z}}\left|\begin{array}{lll}
\varphi_{i+1, j, k}^{1}-\varphi_{i-1, j, k}^{1} & \varphi_{i, j+1, k}^{1}-\varphi_{i, j-1, k}^{1} & \varphi_{i, j, k+1}^{1}-\varphi_{i, j, k-1}^{1} \\
\varphi_{i+1, j, k}^{2}-\varphi_{i-1, j, k}^{2} & \varphi_{i, j+1, k}^{2}-\varphi_{i, j-1, k}^{2} & \varphi_{i, j, k+1}^{2}-\varphi_{i, j, k-1}^{2} \\
\varphi_{i+1, j, k}^{2}-\varphi_{i-1, j, k}^{2} & \varphi_{i, j+1, k}^{2}-\varphi_{i, j-1, k}^{2} & \varphi_{i, j, k+1}^{2}-\varphi_{i, j, k-1}^{2}
\end{array}\right| \\
& =\frac{1}{8 h_{x} h_{y} h_{z}} \sum_{i^{\prime}, j^{\prime}, k^{\prime} \in\{-1,+1\}}\left|\begin{array}{lll}
\varphi_{i+i^{\prime}, j, k}^{1}-\varphi_{i, j, k}^{1} & \varphi_{i, j+j^{\prime}, k}^{1}-\varphi_{i, j, k}^{1} & \varphi_{i, j, k+k^{\prime}}^{1}-\varphi_{i, j, k}^{1} \\
\varphi_{i+i^{\prime}, j, k}^{2}-\varphi_{i, j, k}^{2} & \varphi_{i, j+j^{\prime}, k}^{2}-\varphi_{i, j, k}^{2} & \varphi_{i, j, k+k^{\prime}}^{2}-\varphi_{i, j, k}^{2} \\
\varphi_{i+i^{\prime}, j, k}^{2}-\varphi_{i, j, k}^{2} & \varphi_{i, j+j^{\prime}, k}^{2}-\varphi_{i, j, k}^{2} & \varphi_{i, j, k+k^{\prime}}^{2}-\varphi_{i, j, k}^{2}
\end{array}\right| \\
& =\frac{6}{8 h_{x} h_{y} h_{z}}\left(\mathcal{V}_{\text {odea }}+\mathcal{V}_{\text {oaec }}+\mathcal{V}_{\text {oceb }}+\mathcal{V}_{\text {obed }}+\mathcal{V}_{\text {ocfa }}+\mathcal{V}_{\text {obfc }}+\mathcal{V}_{\text {odfb }}+\mathcal{V}_{\text {oafd }}\right),
\end{aligned}
$$

where $\mathcal{V}_{\text {odea }}$ of the above formulas is the signed volume with the tetrahedron $\mathcal{V}(o, d, e, a)$.

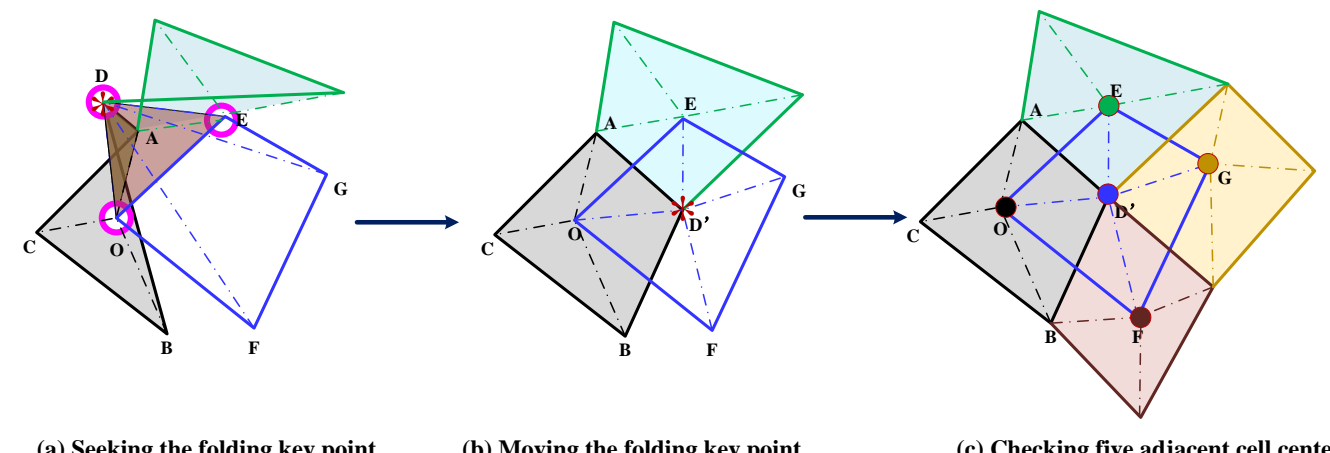

(a) Seeking the folding key point

(b) Moving the folding key point

(c) Checking five adjacent cell centers

FIG. 3. Grid folding correction.

Grid folding correction. Triangulation is widely used in geometric computing, also in image processing community, the unfolding of a single triangle element is the favorable geometric properties of the triangulation that make it so useful. The fundamental property is that a triangle deformation cannot twist without their areas to change sign, such deformation grid $\varphi$ is regular even in cases of large deformations.

However, while the area of a triangle changes sign, the corresponding grid unfolding indicator $R_{i j}$ is negative, and the grid degeneracy arises. To detect the grid degeneracy, we first collect such point $(i, j)$ to set $S:=\left\{(i, j) \mid R_{i j}<0\right\}$ (see three points marked by the magenta circles in Fig.3(a)). Further, we define the folding degree of a point $P$ by

$\operatorname{Deg}_{P}=$ the triangle partition number with a vertex $P$ and the negative area ratio.

to seek the folding key point. Using the above definition, we have that $\mathbf{D e g}_{O}=2, \mathbf{D e g}_{E}=3$ and 
$\operatorname{Deg}_{D}=4$. Hence the point $D$ with large folding degree is selected as a folding key point (see Fig.3(a)).

To correct the grid point $D$ resulting in "twist" of the boxes, the folding key point $D$ is moved to $D^{\prime}$, this movement should ensure that the grid unfolding indicators $\left(R_{O}, R_{E}, R_{G}, R_{F}\right.$ and $\left.R_{D^{\prime}}\right)$ of five adjacent cell centers $\left(O, E, G, F\right.$ and $D^{\prime}$ respectively) are positive. We illustrate this process in Fig.3. The deformation correction algorithm for using the above technique and backtrack strategy is given in Algorithm 1.

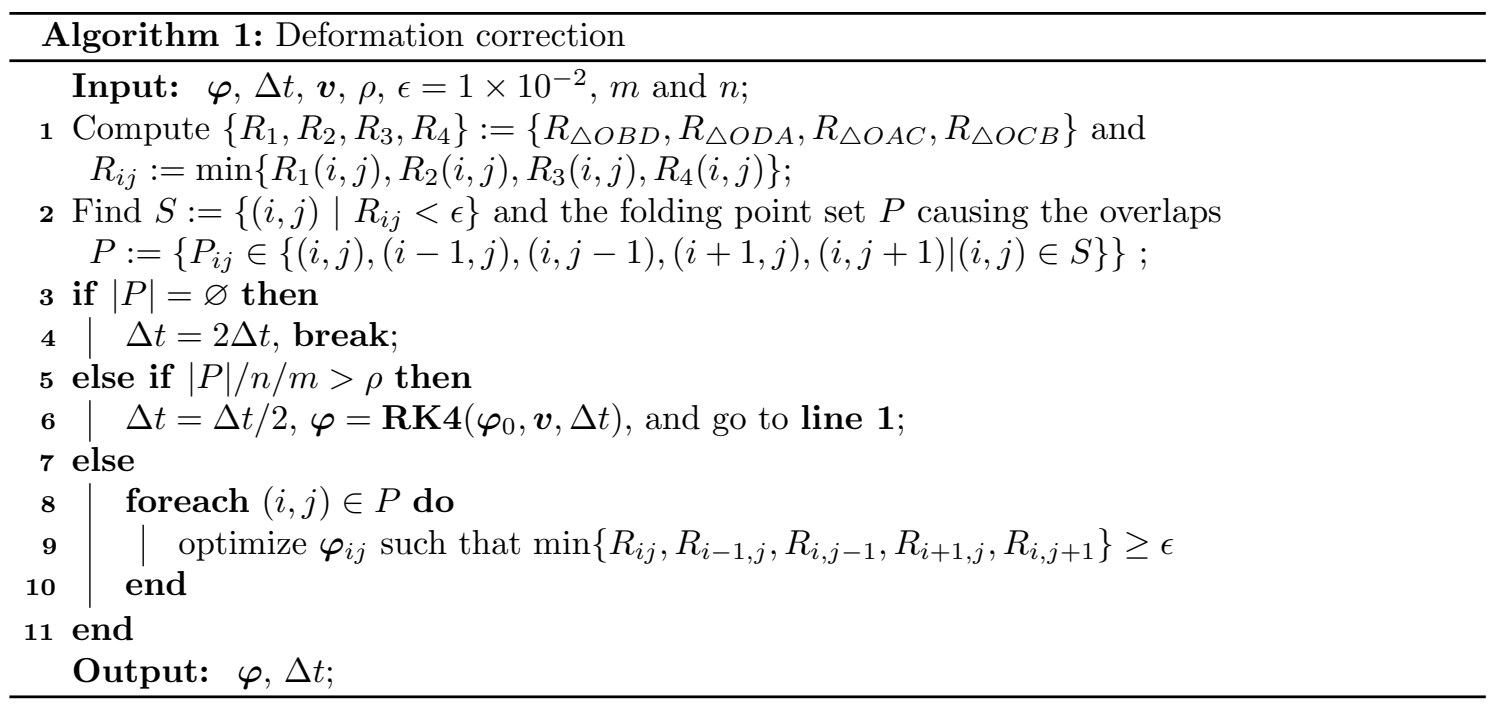

4.5.3. Algorithm. Based on the above discussion, the algorithm based on ALMM solving the proposed diffeomorphic registration model with an optimal control relaxation (ALMM-based OCRDIR) and the deformation correction can be summarized in Algorithm 2.

5. Experimental results. In this section, through numerical experiments, we aim to

i.) select an appropriate composite function $h(\phi(\boldsymbol{x}, t), t)$ of the proposed OCRDIR model;

ii.) compare with the demons-type models to conclude that the new model has better performance;

iii.) compare with the other popular diffeomorphic models to show the diffeomorphism and accuracy of our OCRDIR model for large deformed images.

To assess the performance of our OCRDIR method, we compare

- the qualitative evaluations including the registered images, the errors between the registered image $T(\boldsymbol{\varphi}(\boldsymbol{x}))$ and reference image $R(\boldsymbol{x})$, the deformation grids, the displacement fields and the Jacobian determinant hotmaps of the displacement fields.

- the quantitative evaluations of the deformation $\varphi$ including

a.) Grid unfolding indicator defined by $R_{\min }=\min _{i, j} R_{i j}, R_{\min }>0$ (denoted by "+") indicates geometrically that the grid folding is not detected, while if $R_{\min } \leq 0$ (denoted by "-") means singularity at $\boldsymbol{x}$, the grid folding has occurred;

b.) Jacobian determinant measures defined by

$$
\begin{aligned}
\left.\operatorname{det}(\nabla \boldsymbol{\varphi})\right|_{i j}, & \overline{\operatorname{det}}(J(\boldsymbol{\varphi}))=\left.\frac{1}{m n} \sum_{i, j} \operatorname{det}(\nabla \boldsymbol{\varphi})\right|_{i j}, \\
\operatorname{det}_{\text {min }}(J(\boldsymbol{\varphi}))=\left.\min _{i, j} \operatorname{det}(\nabla \boldsymbol{\varphi})\right|_{i j}, & \operatorname{det}_{\text {max }}(J(\boldsymbol{\varphi}))=\left.\max _{i, j} \operatorname{det}(\nabla \boldsymbol{\varphi})\right|_{i j} .
\end{aligned}
$$




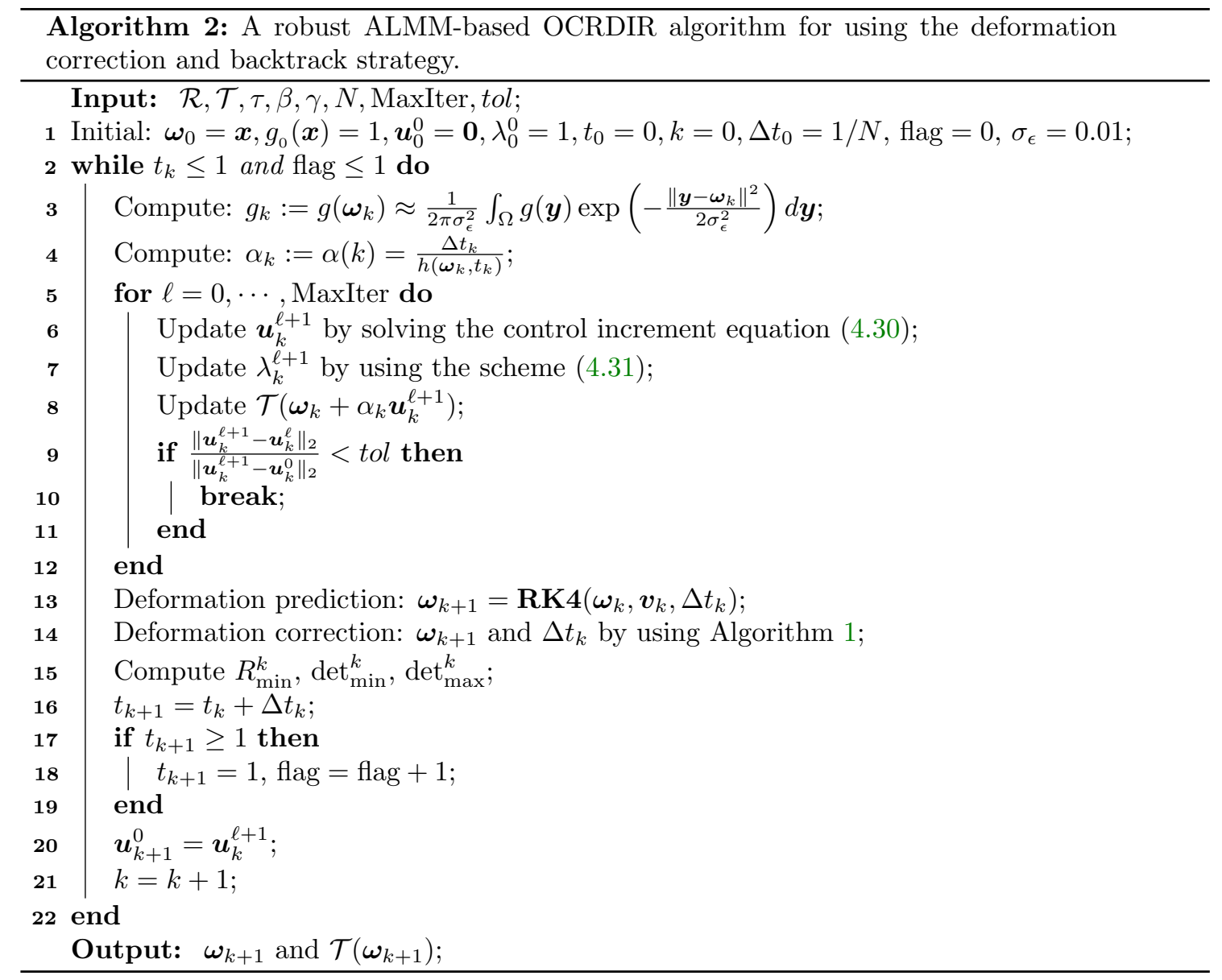

When the grid unfolding indicator $R_{\min }>0$, $\operatorname{det}(J(\varphi))=1$ indicates geometrically that no area (volume) change has occurred, and $\operatorname{det}(J(\varphi))>1$ implies area (volume) growth, and $0<\operatorname{det}(J(\varphi))<1$ indicates shrinkage.

c.) the Re_SSD (the relative Sum of Squared Differences) defined by

$$
\operatorname{Re} \_\operatorname{SSD}(T, R, T(\boldsymbol{\varphi}))=\frac{\int_{\Omega}(T(\boldsymbol{\varphi}(\boldsymbol{x}))-R(\boldsymbol{x}))^{2} d \boldsymbol{x}}{\int_{\Omega}(T(\boldsymbol{x})-R(\boldsymbol{x}))^{2} d \boldsymbol{x}} .
$$

d.) the ssim (Structural Similarity) defined by

$$
\operatorname{sim}(R, T)=\frac{\left(2 \mu_{R} \mu_{T}+c_{1}\right)\left(2 \sigma_{R T}+c_{2}\right)}{\left(\mu_{R}^{2}+\mu_{T}^{2}+c_{1}\right)\left(\sigma_{R}^{2}+\sigma_{T}^{2}+c_{2}\right)},
$$

which is used to measure luminance, contrast and structure between image $R$ and image $T$. Where $\mu_{R}$ and $\mu_{T}$ are the mean of $R$ and $T, \sigma_{R T}$ is the covariance of $R$ and $T, \sigma_{R}^{2}$ and $\sigma_{T}^{2}$ are the variance of $R$ and $T, c_{1}$ and $c_{2}$ are constants. ssim is between 0 and 1 , when the two images are exactly the same, $\operatorname{ssim}=1$.

It should be noted that a good registration should not only generate a small Re_SSD, but also ensure that the grid unfolding indicator $R_{\min }>0$. In an ideal case, we should focus on seeking an 
TABLE 1

Quantitative assessment of different modeling parameters.

\begin{tabular}{|c|c|c|c|c|c|c|}
\hline \multicolumn{2}{|l|}{ Parameter } & $\mathrm{Re}_{-} \mathrm{SSD}$ & $R_{\min }$ & $\operatorname{det}_{\min }(J(\boldsymbol{\varphi}))$ & $\operatorname{det}_{\max }(J(\boldsymbol{\varphi}))$ & $\overline{\operatorname{det}}(J(\varphi))$ \\
\hline \multirow{5}{*}{$\begin{array}{c}\beta=0.01 \\
\gamma=0.01 \\
\tau\end{array}$} & 0.5 & $0.1128 \%$ & + & 0.42 & 2.11 & 1.001 \\
\hline & 1 & $0.0141 \%$ & + & 0.41 & 2.17 & 1.001 \\
\hline & 5 & $0.0086 \%$ & + & 0.40 & 2.16 & 1.001 \\
\hline & 10 & $0.0204 \%$ & + & 0.38 & 2.75 & 1.001 \\
\hline & 20 & $0.0816 \%$ & + & 0.40 & 2.13 & 1.001 \\
\hline \multirow{5}{*}{$\begin{array}{c}\tau=5 \\
\gamma=0.01 \\
\beta\end{array}$} & 0.001 & $0.0086 \%$ & + & 0.40 & 2.16 & 1.001 \\
\hline & 0.01 & $0.0100 \%$ & + & 0.41 & 2.12 & 1.001 \\
\hline & 0.1 & $0.1035 \%$ & + & 0.60 & 2.18 & 1.001 \\
\hline & 1 & $1.5663 \%$ & + & 0.15 & 2.63 & 1.001 \\
\hline & 2 & $2.5158 \%$ & + & 0.15 & 1.63 & 1.001 \\
\hline \multirow{5}{*}{$\begin{array}{c}\tau=5 \\
\beta=0.01 \\
\gamma\end{array}$} & 0.001 & $0.0705 \%$ & + & 0.32 & 2.17 & 1.001 \\
\hline & 0.01 & $0.0086 \%$ & + & 0.40 & 2.16 & 1.001 \\
\hline & 0.1 & $0.0184 \%$ & + & 0.45 & 2.16 & 1.001 \\
\hline & 1 & $0.0221 \%$ & + & 0.51 & 2.17 & 1.001 \\
\hline & 10 & $0.0402 \%$ & + & 0.52 & 2.16 & 1.001 \\
\hline
\end{tabular}

approximation area-preserving deformations $(\overline{\operatorname{det}} \approx 1)$, which can be restricted by the constraint condition.

5.1. Comparison for parameter choices. The purpose of this part is to show how sensitive our approach is with respect to regularization parameter $\tau$, also penalty parameters $\gamma$ and $\beta$. On one hand, a regularization parameter $\tau$ balancing the trade-off between a good SSD metric and a smooth solution is difficult to be fixed: if the value $\tau$ is too large, then it is a poor deformation $\boldsymbol{\varphi}(\boldsymbol{x})$ for matching between $R(\boldsymbol{x})$ and $T(\boldsymbol{\varphi}(\boldsymbol{x}))$, while if too small, the corresponding $\boldsymbol{\varphi}(\boldsymbol{x})$ is not one to one. On the other hand, some experiences on image inverse applications have shown that, if the fixed penalty $\gamma$ or $\beta$ is chosen too small or too large, the solution is worse and the solution time can increase significantly.

We first study how our diffeomorphic image registration model is affected when varying $\tau$. To this end, Algorithm 2 was tested for a synthetic Circle-square image with the results shown in Tab.1, where a pair of $256 \times 256$ synthetic circle images is needed to register into a synthetic square image. The regularization parameter $\tau$ is varied from 1 to 10 in case of fixing $\beta=0.01$ and $\gamma=0.01$. The selection of suitable $\tau$ is important because it is unknown a priori and it significantly affects on the qualities of registered images as well as the algorithm performance [55]. However, for the range of tested in Tab.1, the proposed model still obtains the satisfactory solution in a reasonable range of values $\tau$, so for this example, the accurate selection of $\tau$ is not needed as any $\tau$ between 2 and 10 can give better results, and is reasonable and recommendable.

Next we test how our algorithm with the penalty parameter is affected for the above example when varying the values of $\beta$ and $\gamma ; \beta$ is varied from 0.001 to 0.1 and $\gamma$ is varied from 0.01 to $q$, Tab.1 shows that our model solves the image registration problem. As already discussed previously, the larger $\beta$ will lead to poor matching between $R$ and $T(\varphi)$, while the smaller $\gamma$ will make solution $\varphi$ more worse.

5.2. Comparison of different homotopy composite functions. In this subsection, we briefly give the discussions on the performances of our OCRDIR model by employing different composite functions $\mathrm{P}_{k}(k=1,2,3,4)$. Here the tested Whole-Brain images are of size $128 \times 128$ and the red boxes denote zoom-in regions, we only explain how the proposed model performs when a composite function is used, and see more details about the composite functions in Section 3.2.4.

To make the comparison more fair, we set parameters that make the registration results optimal for different composite functions $\left(\tau=5, \beta=0.01, \gamma=0.01\right.$ for $\mathrm{P}_{1}$ and $\mathrm{P}_{2}, \tau=5, \beta=0.1, \gamma=0.01$ 


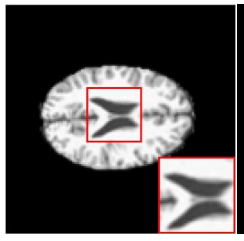

(a) Reference

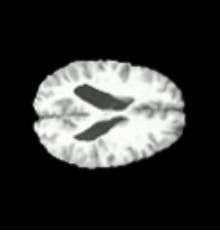

(b) Template

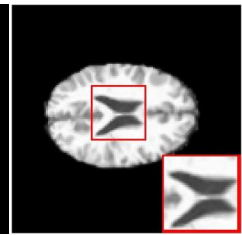

(c) P1

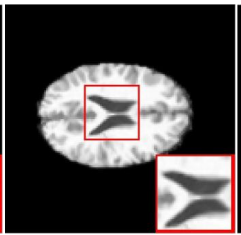

(d) $\mathbf{P 2}$

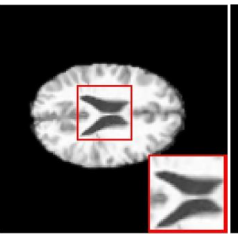

(e) $\mathbf{P 3}$

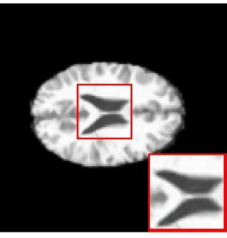

(f) $\mathbf{P 4}$

FIG. 4. Comparison for different composite functions using the Whole-Brain images. From (a) to (f): the reference image with a zoom-in region, template image, four solutions with different composite functions.

for $\mathrm{P}_{3}, \tau=3, \beta=0.1, \gamma=0.01$ for $\left.\mathrm{P}_{4}\right)$. The solutions by using different composite functions based on OCRDIR algorithm are shown in Fig.4 with the reference image and template image shown in Fig.4 (a) and Fig.4 (b), four registered images from $T$ to $R$ are presented in Fig.4 (c)-(f) for taking the time-step length $\Delta t=1 / N(N=40)$, respectively. We can see that all four models can produce visually satisfactory results.

TABLE 2

Quantitative assessment of different composite functions for the proposed OCRDIR model, where 'CF' means to use different homotopy composite function.

\begin{tabular}{cccccccc}
\hline $\mathbf{C F}$ & $\overline{\operatorname{det}}(J(\boldsymbol{\varphi}))$ & $R_{\text {min }}$ & $\operatorname{det}_{\text {min }}(J(\boldsymbol{\varphi}))$ & $\operatorname{det}_{\text {max }}(J(\boldsymbol{\varphi}))$ & ssim & $\mathrm{Re}_{-}$SSD & psnr \\
\hline $\mathbf{P}_{1}$ & 1.000 & + & 0.13 & 3.17 & 0.9900 & $2.06 \%$ & 27.94 \\
\hline $\mathbf{P}_{2}$ & 1.000 & + & 0.15 & 3.17 & 0.9900 & $2.08 \%$ & 27.94 \\
\hline $\mathbf{P}_{3}$ & 1.001 & + & 0.16 & 3.92 & 0.9865 & $2.62 \%$ & 26.70 \\
\hline $\mathbf{P}_{4}$ & 1.000 & + & 0.16 & 3.18 & 0.9897 & $2.07 \%$ & 27.94 \\
\hline
\end{tabular}

In Tab.2, the grid unfolding indicator $R_{\min }>0$ shows that the four composite functions can ensure the diffeomorphism of the deformation. Further we can also see that all $\overline{\text { det values }}$ for the four composite functions approach to 1 , which indicates that the deformations are area preserving for 2D case. The Jacobian determinants of the four composite functions all belong to a small interval $\left[\operatorname{det}_{\min }, \operatorname{det}_{\max }\right]$, which means that the degree of area change of deformed grid is considerably small, because the new registration model explicitly controls and penalizes area change by Jacobian equation. Furthermore, $\mathrm{P}_{3}$ in this example is not as good as $\mathrm{P}_{1}, \mathrm{P}_{2}$ and $\mathrm{P}_{4}$ because it gives worse ssim, Re_SSD and psnr, which means the registered images obtained by the composite function have significant registration errors between the reference and template images. So we recommend the composite function $\mathrm{P}_{1}$ as the used composite function in our model and in the following numerical experiments.

5.3. Registration with affine preprocessing. Deformable registration is applicable to a large class of non-rigid registration problems, while it can suffer from worse mesh distortion for solving the large rotate deformation problems if the boundary of transforming grid is fixed, e.g., Dirichlet boundary condition $\boldsymbol{u}=\mathbf{0}$ on $\partial \Omega$. Affine image registration is one of the commonly-used rigid parametric models. Such affine method is always many orders of magnitude faster than a nonlinear variational method due to much less unknowns involved, which is widely used as a preregistration step for sophistical non-rigid registration methods, such as elastic, fluid, and diffusion registration, by providing the good initial positions for the image to be registered.

To show the quantitative gain from using two registration steps: pre-registration step and non-rigid registration, we now present the comparable results in Tab.3 for clarity, where the grid 


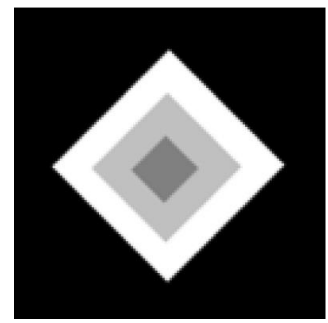

(a) Reference

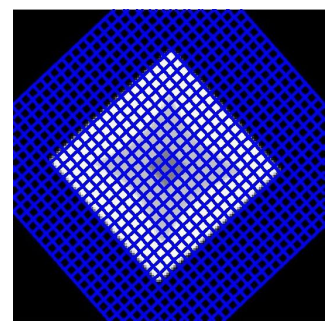

(e) Affine-transformation

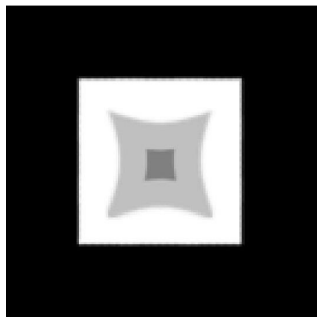

(b) Template

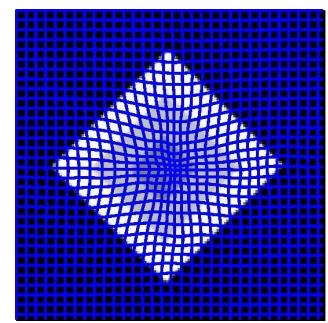

(f) Local transformation

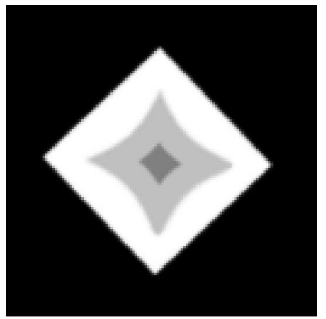

(c) Affine pre-registration

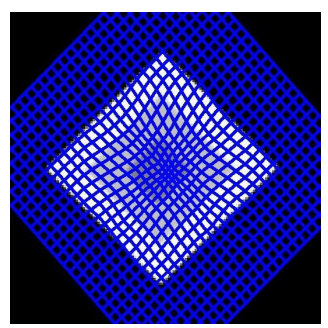

(g) Global transformation

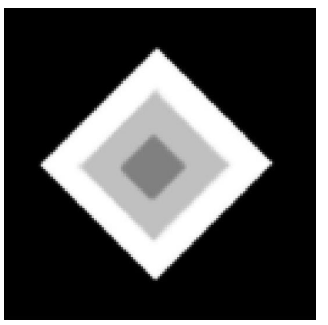

(d) Local registration

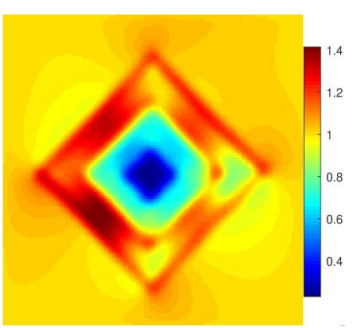

(h) Global determinant

FIG. 5. Registration with affine preprocessing. (a) the reference image; (b) the template image; (c) the preregistration results of the affine model; (d) the proposed local registration from the template (c); (e) the affine large deformation grid; $(f)$ the proposed local deformation grid; $(g)$ the global deformation grid from the template (b) to the reference $(a)$; ( $h$ ) the Jacobian determinant hotmaps of global deformation $\varphi$.

TABLE 3

Registration with affine preprocessing.

\begin{tabular}{cccccccc}
\hline$\overline{\operatorname{det}(J(\varphi))}$ & $R_{\text {min }}$ & $\operatorname{det}_{\text {min }}(J(\varphi))$ & $\operatorname{det}_{\text {max }}(J(\varphi))$ & ssim & $\mathrm{Re}_{-} \mathrm{SSD}$ & psnr & runtime $(\mathrm{s})$ \\
\hline 0.9998 & + & 0.21 & 1.44 & 0.9974 & $0.28 \%$ & 39.54 & 15.17 \\
\hline
\end{tabular}

unfolding indicator $R_{\min }>0$ and $\operatorname{det}(J(\varphi)) \in[0.21,1.44]$ indicate that the registration keeps the diffeomorphism. It is worth noting that the Re_SSD and psnr are $0.28 \%$ and 39.54 respectively, and the ssim is as high as 0.9974 . We also show the respective registration visualizations with affine preprocessing in Fig.5.

5.4. Comparison of the new model with Demons-type models. Active demons algorithm is an automatic and efficient registration algorithm, which uses all the image information to avoid the error caused by artificial intervention. It also has certain advantages in image registration when the reference image gradient is very small and the deformation $\varphi$ is relatively large. It is an important algorithm in image registration. Log-Demons algorithm inherits the ability of the demons algorithm in dealing with large deformation registration, it also can keep diffeomorphism of the deformation $\boldsymbol{\varphi}$. So we compare with the demons-type models to conclude that the new model has better performance for small or large deformation. In this example, we test three pairs of images, including Synthetic images, Lena images and Cardiac images of resolution $128 \times 128$. We compare the proposed OCRDIR model with the active demons and diffeomorphic log-demons to demonstrate the performance of our model.

The proposed ALMM-based OCRDIR algorithm is to solve a time-dependent optimization problem, where the maximum number of outer iterations of time-step $t_{j}$ is set to $N=40$ (i.e., 

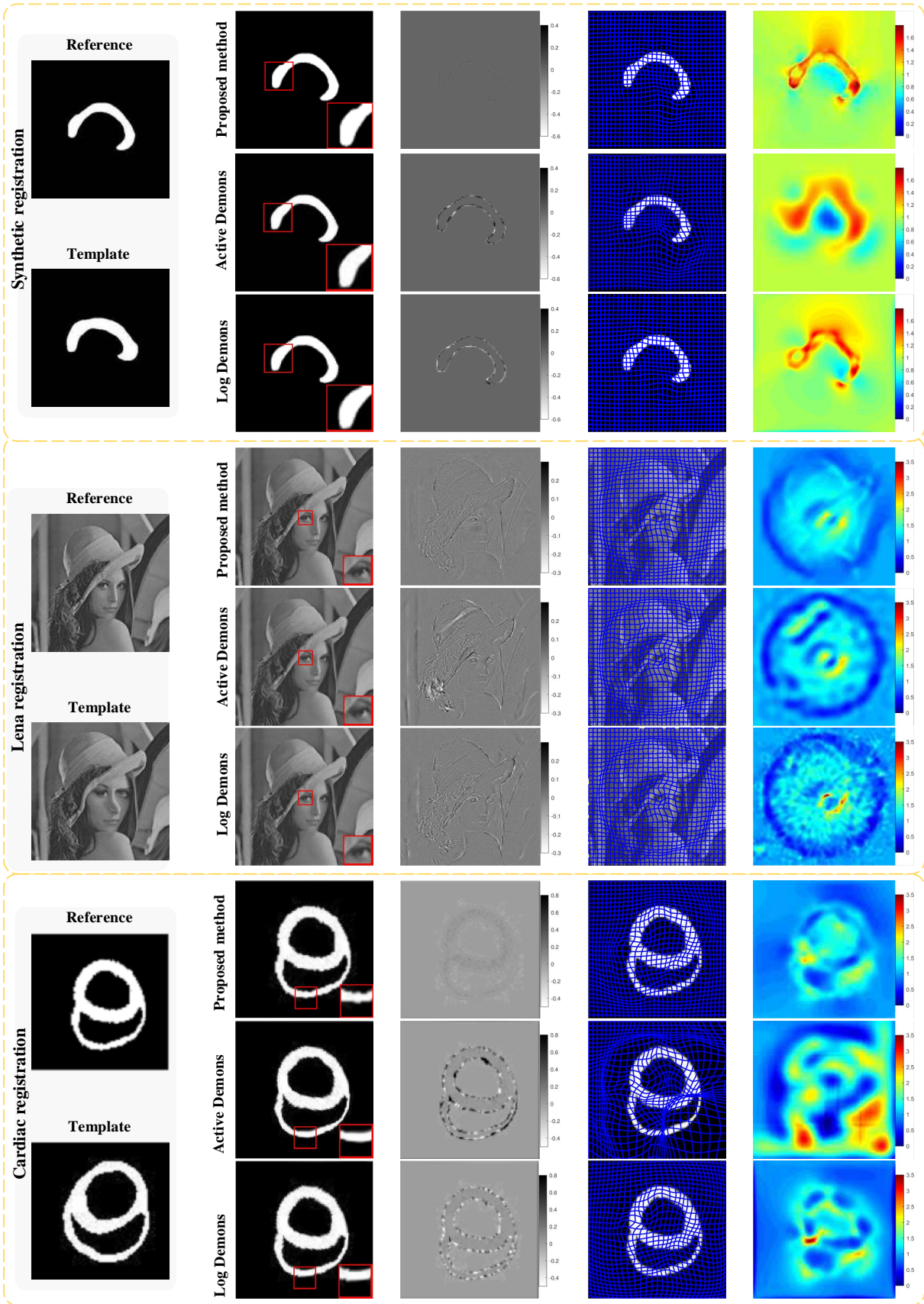

(a) Examples

(b) Registered

(c) Error

(d) Deformation grid

(e) Determinant

FiG. 6. Comparison with active demons and diffeomorphic log-Demons. (a) the reference and template images (Synthetic, Lena and Cardiac examples); (b) the registered results of the three classical models with the optimal parameters (Synthetic: $\tau=3, \beta=0.01, \gamma=0.1$ and $N=40$ for OCRDIR model, Gaussian filter $\sigma^{2}=10, \tau=0.8$ and MaxIter $=200$ for active demons model, $\sigma_{\text {fluid }}=1, \sigma_{\text {diffusion }}=1, \sigma_{i}=2, \sigma_{x}=2$, nlevel $=5$ and niter $=40$ for Log-Demons model; Lena: $\tau=3, \beta=0.1, \gamma=0.5$ and $N=40$ for OCRDIR model, Gaussian filter $\sigma^{2}=6, \tau=2.4$ and MaxIter $=200$ for active demons model, $\sigma_{\text {fluid }}=1, \sigma_{\text {diffusion }}=1, \sigma_{i}=2, \sigma_{x}=2$, nlevel $=5$ and niter $=40$ for Log-Demons model; Cardiac: $\tau=2.5, \beta=0.05, \gamma=0.01$ and $N=60$ for the proposed model, Gaussian filter $\sigma^{2}=10, \tau=0.4$ and MaxIter $=300$ for active demons model, $\sigma_{\text {fluid }}=4, \sigma_{\text {diffusion }}=1, \sigma_{i}=2, \sigma_{x}=2$, nlevel $=5$ and niter $=60$ for Log-Demons model.); (c) registration errors $T(\boldsymbol{\varphi})-R(\boldsymbol{x}) ;(d)$ the deformation grids; (e) the Jacobian determinant hotmaps of the displacement fields. 
TABLE 4

Comparison of the new model with Demons

\begin{tabular}{ccccccccc}
\hline Example & Method & $\overline{\operatorname{det}}(J(\boldsymbol{\varphi}))$ & $R_{\min }$ & $\min \operatorname{det}(J(\boldsymbol{\varphi}))$ & $\max \operatorname{det}(J(\boldsymbol{\varphi}))$ & ssim & $\mathrm{Re}_{\mathrm{SSD}}$ & psn $\boldsymbol{r}$ \\
\hline \multirow{3}{*}{ Synthetic } & Proposed & 1.000 & + & 0.45 & 1.95 & $\mathbf{1 . 0 0 0 0}$ & $\mathbf{0 . 0 0 1} \%$ & $\mathbf{5 2 . 1 9}$ \\
& Active Demons & 1.008 & + & 0.31 & 1.82 & 0.9949 & $1.50 \%$ & 19.65 \\
& Log Demons & 1.015 & + & 0.64 & 1.60 & 0.9932 & $1.70 \%$ & 19.55 \\
\hline \multirow{2}{*}{ Lena } & Proposed & 1.001 & + & 0.48 & 2.58 & 0.9505 & $\mathbf{3 . 7 8} \%$ & $\mathbf{2 6 . 8 4}$ \\
& Active Demons & 1.079 & + & 0.30 & 2.37 & 0.9067 & $9.50 \%$ & 22.91 \\
& Log Demons & 1.072 & + & 0.29 & 3.52 & 0.9330 & $7.12 \%$ & 24.36 \\
\hline \multirow{2}{*}{ Cardiac } & Proposed & 1.001 & + & 0.33 & 2.96 & 0.8369 & $\mathbf{1 . 0 6} \%$ & $\mathbf{2 1 . 0 1}$ \\
& Active Demons & 1.539 & - & $\mathbf{- 0 . 0 2}$ & 3.09 & $\mathbf{0 . 8 4 5 9}$ & $6.21 \%$ & 13.48 \\
& Log Demons & 1.107 & + & 0.09 & 3.52 & 0.8205 & $3.38 \%$ & 16.18 \\
\hline
\end{tabular}

$\left.t_{j} \in[0,1], j=1, \cdots, N\right)$, the maximum number of inner iterations of our algorithm is set to MaxIter $=5$ and the tolerance for the relative residual is set to $10^{-6}$. To make a fair comparison in implementation, we set the iteration of active demons algorithm to $\mathrm{N} \times$ MaxIter as well. The diffeomorphic log-demons uses a multi-resolution strategy for better registration, we let the level of multi-resolution be nlevel $=5$, niter $=$ MaxIter.

In Synthetic and Lena examples, Fig.6 shows that all three methods can produce satisfactory visualizations. The proposed method can present more image details which can be seen from the zoom-in images (see Fig.6(b)), and obtain better registration performances, which not only is qualitatively shown by visual inspection of the image mismatch errors (see Fig.6(c)), but also is quantitatively analyzed. As has been seen, the Re_SSD, ssim and psnr of the new model are better than active demons and diffeomorphic log-demons, where the ssim and psnr reach 1.000 and 52.19 respectively, and the Re_SSD is as low as $0.001 \%$. For two examples with small deformation in Fig.6(d), all models can generate diffeomorphic deformations and obtain smooth grid. It also can be seen from Tab. 4 that all models produce the grid unfolding indicator $R_{\min }>0$ in Synthetic and Lena examples, i.e. the deformations obtained by these three models are diffeomorphic. We can notice that the transformed grids generated by the three models are relatively smooth for the small deformation, but the transformed grids obtained by active demons are winding for the large deformation obviously. Fig.6(e) shows the hotmaps of the Jacobian determinant, where the right color bars illustrate the range of the Jacobian determinant values at each pixel $\boldsymbol{x}$ (see Tab.4 for more details).

However, for large deformation example in Cardiac in Fig.6, it is known from Tab.4 that the deformation obtained by the active demons is non-diffeomorphic due to the grid unfolding indicator $R_{\min }<0$, i.e. mesh folding. From Fig.6(d), we can also see that the diffeomorphic log-demons generates diffeomorphic transformation, but the ssim, Re_SSD and psnr are worse in Tab.4.

Therefore, those examples demonstrate that our OCRDIR model can get an accurate and diffeomorphic transformation for both small and large deformation registrations.

5.5. Comparison of the new model with other diffeomorphic models. In this experiment, we compare our OCRDIR model with three popular diffeomorphic image registration models. The first compared approach is naturally the Hsiao's model proposed by H. Y. Hsiao [28], which uses div-curl system as constraints to generate smooth and invertible deformation. The second and third compared works are the efficient LDDMM frameworks (with diffusion regularizer and curvature regularizer, respectively) [38], they can efficiently solve large deformation diffeomorphic registration problems. Their approaches are different from ours since they are focused on grid generation and a multi-resolution technique. For the LDDMM framework we use a five-level strategy in this experiment, the values for the regularization parameters are optimally chosen, and other parameters refer to default values provided in LDDMM framework to solve the problem. 

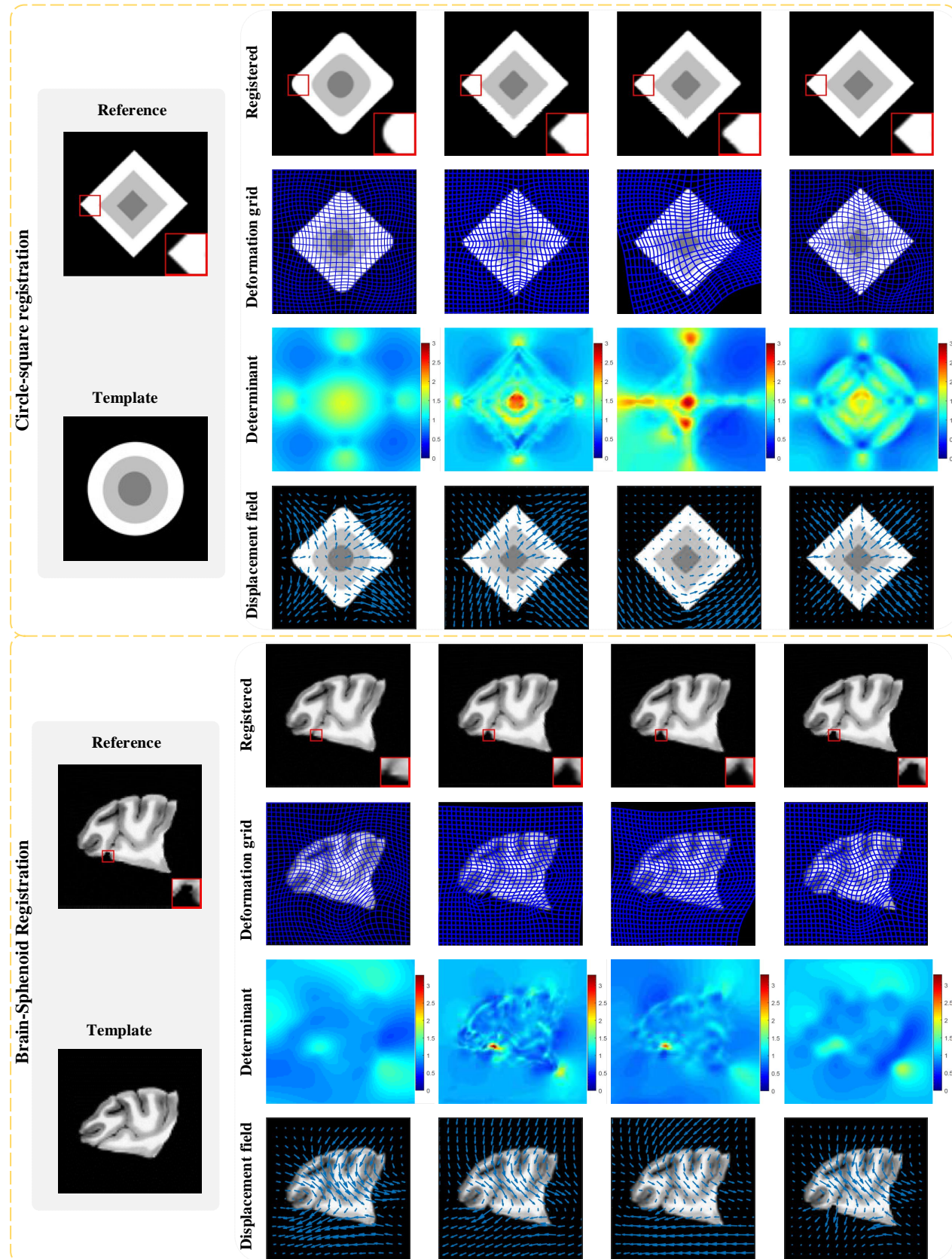

(a) Examples
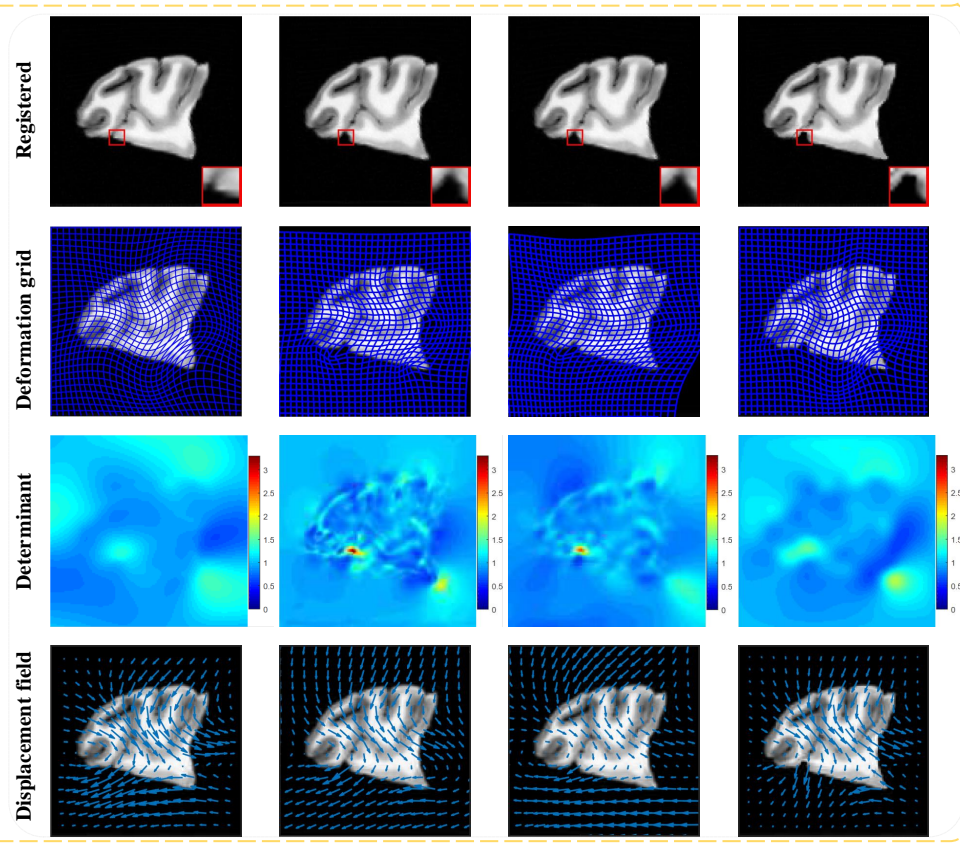

(c) LDDMM Diff

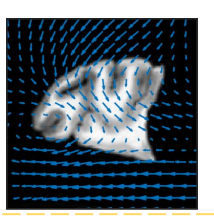

(d) LDDMM Curv

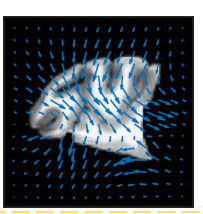

(e) Proposed method

Fig. 7. Comparison with three diffeomorphic variational models for Circle-square and Brain-sphenoid examples. (a) shows reference and template images; (b)-(e) show the results of H. Y. Hsiao'model, LDDMM diffusion model, LDDMM curvature model and the proposed OCRDIR model; from top to bottom: the registered images, the deformation grids, the Jacobian determinant hotmaps of the deformation grids and the displacement fields, respectively. The parameters are chose as follows: $\alpha=80, \alpha=0.1$ for LDDMM-Diff and LDDMM-Curv respectively, $\tau=4, \beta=0.01, \gamma=0.01$ and $N=40$ for the proposed model in Circle-square test; $\alpha=100, \alpha=0.1$ for LDDMM-Diff and LDDMM-Curv respectively, $\tau=4, \beta=0.05, \gamma=0.01$ and $N=40$ for the proposed model in Brain-sphenoid example. 


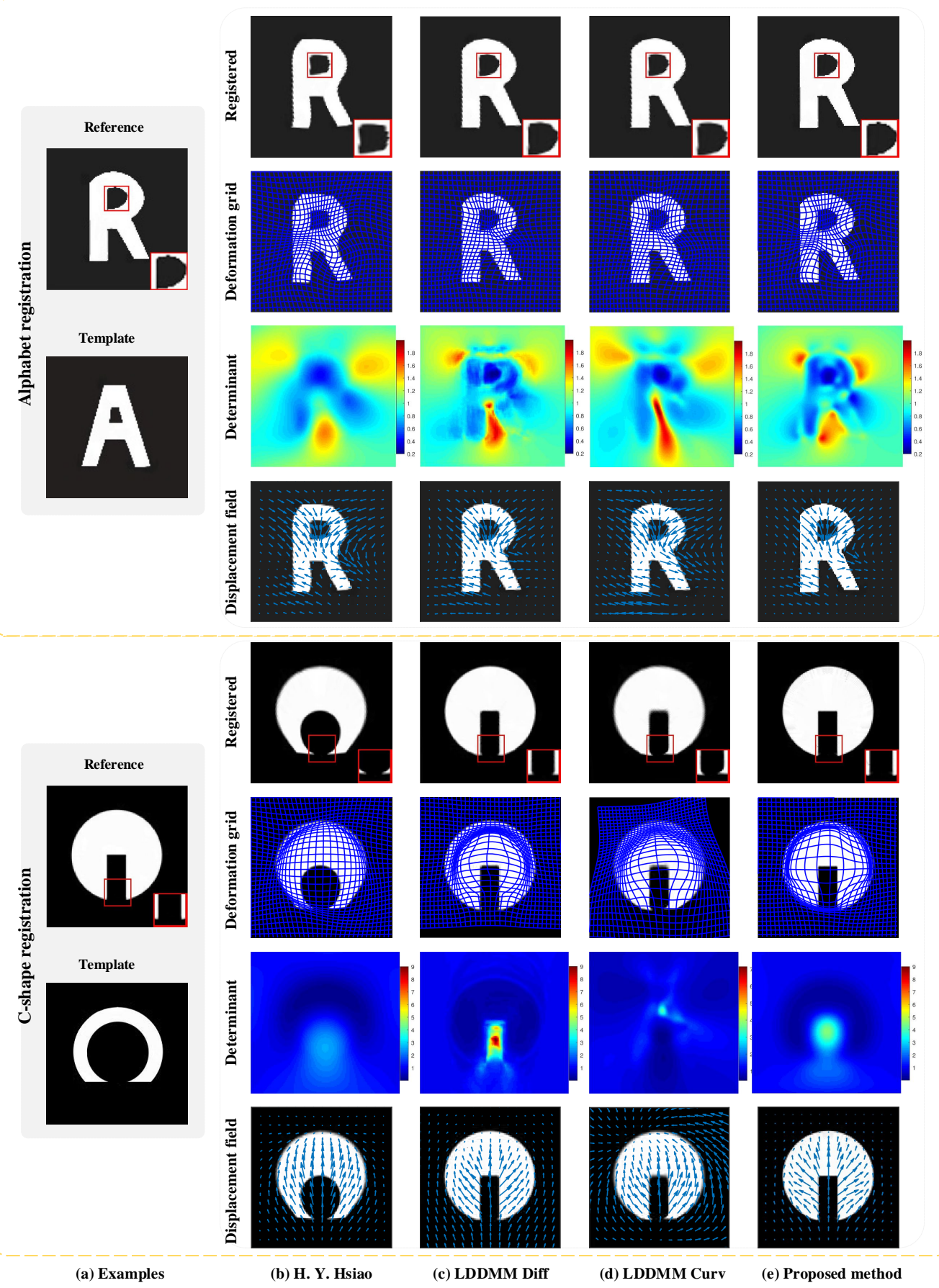

FIG. 8. Comparison with three diffeomorphic variational models for large deformation image pairs: Alphabet and C-shape examples. (a) shows reference and template images; (b)-(e) show the diffeomorphic registration results of H. Y. Hsiao's model, LDDMM diffusion model, LDDMM curvature model and the proposed OCRDIR model; from top to bottom: the registered images, the deformation grids, the Jacobian determinant hotmaps of the deformation grids and the displacement fields. The parameters are chose as follows: $\alpha=300, \alpha=1$ for LDDMM-Diff and LDDMM-Curv respectively, $\tau=4, \beta=0.01, \gamma=1$ and $N=40$ for the proposed model in Alphabet example; $\alpha=200, \alpha=10$ for LDDMM-Diff and LDDMM-Curv respectively, $\tau=3, \beta=0.01, \gamma=0.05$ and $N=60$ for the proposed model in C-shape test. 
TABLE 5

Comparison of the new OCRDIR model with Hsiao's model and LDDMM frameworks (with the diffusion regularizer and the curvature regularizer).

\begin{tabular}{|c|c|c|c|c|c|c|c|c|c|c|}
\hline Example & Method & $\overline{\operatorname{det}}(J(\varphi))$ & $R_{\text {min }}$ & $\operatorname{det}_{\min }(J(\boldsymbol{\varphi}))$ & $\operatorname{det}_{\max }(J(\boldsymbol{\varphi}))$ & ssim & $\mathrm{Re}_{-} \mathrm{SSD}$ & psnr & Runtime(s) & $\operatorname{Memory}(\mathrm{Mb})$ \\
\hline \multirow{4}{*}{$\begin{array}{l}\text { Brain- } \\
\text { sphenoid }\end{array}$} & Proposed & 1.000 & + & 0.24 & 2.77 & 0.9622 & $0.44 \%$ & 28.31 & 10.15 & 16.06 \\
\hline & Hsiao & 1.030 & + & 0.61 & 1.43 & 0.9195 & $5.10 \%$ & 17.72 & 30.44 & 9.25 \\
\hline & LDDMM-Diff & 0.971 & + & 0.51 & 3.22 & 0.9444 & $0.94 \%$ & 24.99 & 132.03 & 20.29 \\
\hline & LDDMM-Cur & 0.920 & + & 0.56 & 2.66 & 0.9459 & $1.02 \%$ & 24.67 & 205.55 & 40.68 \\
\hline \multirow{4}{*}{$\begin{array}{l}\text { Circle- } \\
\text { square }\end{array}$} & Proposed & 1.001 & + & 0.34 & 2.15 & 0.9994 & $0.008 \%$ & 45.58 & 15.21 & 16.23 \\
\hline & Hsiao & 1.061 & + & 0.65 & 1.74 & 0.9372 & $3.60 \%$ & 19.02 & 30.30 & 11.82 \\
\hline & LDDMM-Diff & 1.079 & + & 0.56 & 2.54 & 0.9892 & $0.76 \%$ & 25.83 & 196.49 & 20.44 \\
\hline & LDDMM-Curv & 1.085 & + & 0.49 & 2.85 & 0.9872 & $0.87 \%$ & 25.26 & 226.01 & 42.71 \\
\hline \multirow{4}{*}{ Cardiac } & Proposed & 1.001 & + & 0.33 & 2.96 & 0.8369 & $1.04 \%$ & 21.01 & 15.97 & 12.44 \\
\hline & Hsiao & 1.073 & + & 0.60 & 2.45 & 0.7854 & $5.28 \%$ & 14.20 & 45.58 & 10.83 \\
\hline & LDDMM-Diff & 1.084 & + & 0.26 & 3.85 & 0.8347 & $1.69 \%$ & 19.13 & 221.80 & 21.23 \\
\hline & LDDMM-Curv & 1.115 & + & 0.52 & 2.92 & 0.8094 & $3.26 \%$ & 16.29 & 414.74 & 39.61 \\
\hline \multirow{4}{*}{ A-R } & Proposed & 0.999 & + & 0.20 & 1.87 & 0.9949 & $0.05 \%$ & 36.82 & 11.16 & 11.29 \\
\hline & Hsiao & 1.047 & + & 0.40 & 1.53 & 0.9392 & $8.40 \%$ & 14.93 & 59.49 & 10.23 \\
\hline & LDDMM-Diff & 1.010 & + & 0.20 & 1.87 & 0.9820 & $1.58 \%$ & 22.11 & 375.95 & 24.50 \\
\hline & LDDMM-Curv & 1.013 & + & 0.26 & 2.00 & 0.9778 & $2.10 \%$ & 20.89 & 406.23 & 45.04 \\
\hline
\end{tabular}

We start by giving illustrative registered reconstructions in Fig.7, which clearly shows the advantage of using dynamical system over the LDDMM frameworks (with diffusion regularizer and curvature regularizer, respectively) and the Hsiao's model. As is shown in the first row of Fig.7, our method perfectly registers the sharp edges of the large deformation images, the registered images are almost the ground truth reference images. On the other hand, the Hsiao's model just registers the smooth faces, while the LDDMM can register almost the ground truth references except some place "under-enhancement". The second and third rows of Fig.7 shows the transformations and hotmaps of Jacobian determinant $\operatorname{det}(J(\varphi))$ by four models. In the fourth row of Fig.7, we see that our method produces the best displacement fields or optical flows, which shows a major advantage (or better performance) of our proposed model when the displacement between the template and reference images is of large deformation, and even when the contrast between meaningful objects and the background is low. But in the LDDMM framework with curvature regularization, the deformation grids are obviously distorted. Besides, the Jacobian hotmap has a large range of changes.

To present more quantitative differences, we compare all four models by computing the grid unfolding indicator $R_{\min }$, the average value $\overline{\operatorname{det}}(J(\varphi))$, minimizer $\operatorname{det}_{\min }(J(\varphi))$ and maximizer $\operatorname{det}_{\max }(J(\varphi))$ of Jacobian determinant $\operatorname{det}(J(\varphi))$, also compare the ssim, Re_SSD and psnr values between the registered image and reference image, respectively. We show the results in Tab.5. Here all models can generate diffeomorphic solutions as judged by the values of the grid unfolding indicator $R_{\min }$. While the range of the relative area change is considerably smallest of the Hsiao's model $(\operatorname{det}(J(\varphi)) \in[0.61,1.43]$ and $\operatorname{det}(J(\varphi)) \in[0.65,1.74])$ among the four methods, which leads to over-preservation of area, hence it give worse Re_SSD, ssim and psnr. The LDDMM model with the diffusion regulariser leads to further improvements over the Hsiao's model but exhibits the bigger mesh change near large deformation region (e.g., $\operatorname{det}(J(\varphi)) \in[0.51,3.22]$ for Brain-Sphenoid example). The proposed method leads to significantly better results, the range of the relative area change is considerably smaller than whose of the LDDMM, e.g., $\operatorname{det}(J(\varphi)) \in[0.24,2.77]$, $\operatorname{det}(J(\varphi)) \in[0.34,2.15]$ for the Brain-sphenoid, Circle-square, respectively. In addition, in the Brain-sphenoid and Circle-square examples, our model generates better Re_SSD, ssim and psnr than the LDDMM. Especially in the Circle-square experiment, our model obtains the best Re_SSD of $0.008 \%$, ssim of 0.9994 and psnr of 45.58 . This means that the deformed image is exactly the same as the reference image. 
Comparison for the $3 D$ experiments.

\begin{tabular}{cccccccc}
\hline Example & $\overline{\operatorname{det}}(J(\boldsymbol{\varphi}))$ & $R_{\min }$ & $\operatorname{det}_{\min }(J(\boldsymbol{\varphi}))$ & $\operatorname{det}_{\max }(J(\boldsymbol{\varphi}))$ & ssim & $\mathrm{Re}_{-} \mathrm{SSD}$ & $\boldsymbol{p s n \boldsymbol { r }}$ \\
\hline 3D-Brain & 0.9992 & + & 0.06 & 4.23 & 0.9215 & $0.34 \%$ & 18.71 \\
\hline
\end{tabular}

This is due to the fact that our model explicitly controls and penalizes area change by the control increment constraint with time evolution and its regularization (4.7). The reason is that the new OCRDIR model tries to approximate Jacobian determinant $\operatorname{det}(J(\phi(\boldsymbol{x}, t))) \approx 1$ of $\boldsymbol{\phi}(\boldsymbol{x}, t)$ based on high order smooth regulariser, which is clearly better in this case; in other words, our approach is more effective in eliminating the error $T(\boldsymbol{\phi}(\boldsymbol{x}, t))-R(\boldsymbol{x})$ for smooth deformation and is competitive with high order methods. Hence, those examples demonstrate that our model has advantages over other models for large deformation registrations and can all help to get an accurate deformed image and diffeomorphic transformation.

At the end of this part, we perform the registration by Alphabet pairs and $C$-shape pairs with larger deformation by the popular diffeomorphic models and our OCRDIR model. The registered images, the deformation grids, the Jacobian determinant hotmaps of the deformation grids and the displacement fields are shown in Fig.8. The both experiments show that our algorithm can deal with the details better.

5.6. 3D registration experiment. In [7], the cofactor matrix is employed to quantify surface changes, where the solution is defined by

$$
\begin{aligned}
\mathcal{A}_{0}:=\{y & \in W^{1,2}\left(\Omega, \mathbb{R}^{3}\right): \\
& \left.\quad \operatorname{cof} \nabla y \in L_{4}\left(\Omega, \mathbb{R}^{3 \times 3}\right), \operatorname{det} \nabla y \in L_{2}(\Omega, \mathbb{R}), \operatorname{det} \nabla y>0 \text { a.e. }\right\} .
\end{aligned}
$$

The existence theory of diffeomorphic solutions of registration problem using standard arguments from the theory of the hyperelastic regularization is build in their work [7]. The solution properties of our proposed model has been discussed in the previous Section, here we focus on a most challenging large deformation registration.

In this part, we will conduct two registration experiments with $128 \times 128 \times 128$ brain images and $64 \times 64 \times 64$ synthetic images to verify the effectiveness of the proposed approach for $3 \mathrm{D}$ image registration. The relevant parameters in the experiment are fixed as follows: $N=20$, MaxIter $=5, \tau=5, \beta=0.01, \gamma=1$. The experimental results are shown in Fig.9, and the performance indicators are list in Tab.6.

3D results shows the significance and the effect of using the proposed approach. Carefully comparing Figs.9(b)-(e), we notice that our new model deals with the brain and synthetic images better than the other three models, especially in reducing the dissimilarity.

6. Conclusion. Maintaining the continuity and reversibility of the deformation field is a key issue in image registration modeling, and the deformation with diffeomorphism just meets this requirement. In this paper, the proposed OCRDIR model is based on the Jacobian equation with diffeomorphic solution as a constraint condition. Combined with the theoretical proof, it is known that the new OCRDIR model can always ensure the diffeomorphism of the deformation field during the registration. We analyzed an efficient ALMM scheme to efficiently solve the relaxation optimization problem arising in our OCRDIR model. We gave a grid unfolding indicator for cell central-difference discretization, then a robust ALMM-based OCRDIR algorithm for using the deformation correction and backtrack strategy is proposed to guarantee that the solution is diffeomorphic. The numerical results demonstrate that our ALMM-based OCRDIR algorithm can efficiently register both small and large deformation image registration. Our future work will focus 


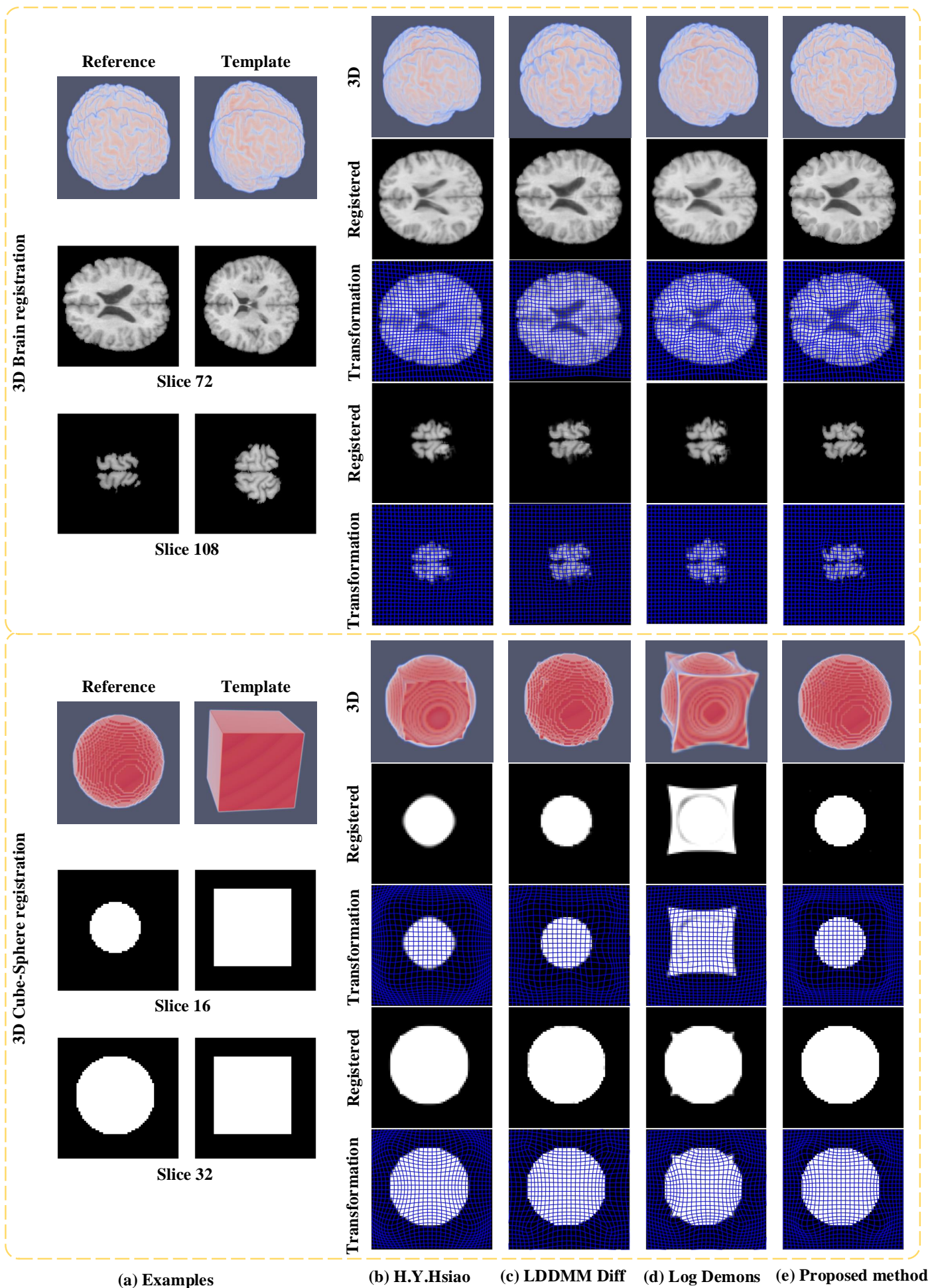

FIG. 9. Comparison of $3 D$ registration. (a) the reference images and template images of $3 D$ Brain and CubeSphere examples, as well as different slices; (b)-(e) the experimental results of H. Y. Hsiao, LDDMM Diff, Log Demons and the proposed method, respectively. 
on the development of faster optimization algorithm and the registration model in multi-modality diffeomorphic image registration.

\section{REFERENCES}

[1] E. L. Allgower And K. Georg, Continuation and path following, Acta Numerica, 2 (1993), pp. 1-64.

[2] R. BAJCsy and S. Kovacic, Multiresolution elastic matching, Graphical Models graphical Models and Image Processing computer Vision, Graphics, and Image Processing, 46 (1989), pp. 1-21.

[3] H. H. Bauschke and P. L. Combettes, Fejér Monotonicity and Fixed Point Iterations, Springer New York, New York, NY, 2011, pp. 75-86.

[4] M. F. Beg, M. Miller, A. Trouve, and L. Younes, Computing large deformation metric mappings via geodesic flows of diffeomorphisms, International Journal of Computer Vision, 61 (2005), pp. 139-157.

[5] A. Behan, 2d and 3d image registration for medical, remote sensing, and industrial applications., Photogrammetric Record, 21 (2006), pp. 180-181.

[6] C. Broit, Optimal Registration of Deformed Images, PhD thesis, USA, 1981.

[7] M. Burger, J. Modersitzki, And L. Ruthotto, A Hyperelastic Regularization Energy for Image Registration, 35, pp. B132-B148.

[8] P. Cachier, X. Pennec, And N. Ayache, Fast non rigid matching by gradient descent: Study and improvements of the demons algorithm, (1999).

[9] M. Carl, L. Galeotti, and B. Löwe, The bolzano-weierstrass theorem in generalised analysis, Houston Journal of Mathematics, 44 (2018), pp. 1081-1109.

[10] C. Chen, B. Gris, And O. OKtem, A new variational model for joint image reconstruction and motion estimation in spatiotemporal imaging, SIAM Journal on Imaging Sciences, 12 (2019), pp. 1686-1719.

[11] C. Chen And O. OKTEM, Indirect image registration with large diffeomorphic deformations, SIAM Journal on Imaging Sciences, 11 (2018), pp. 575-617.

[12] X. Chen, Numerical Construction of Diffeomorphism and the Applications to Grid Generation and Image Registration, PhD thesis, USA, 2016.

[13] G. E. Christensen, S. Joshi, and M. Miller, Volumetric transformation of brain anatomy, IEEE Transactions on Medical Imaging, 16 (1997), pp. 864-877.

[14] G. E. Christensen, R. D. Rabbitt, and M. I. Miller, Deformable templates using large deformation kinematics, Trans. Img. Proc., 5 (1996), pp. 1435-1447.

[15] B. Dacorogna And J. Moser, On a partial differential equation involving the jacobian determinant, Annales De L Institut Henri Poincare-analyse Non Lineaire, 7 (1990), pp. 1-26.

[16] B. Fischer and J. Modersitzki, Fast diffusion registration, Contemporary Mathematics, 313 (2002), pp. 117128.

[17] _ Curvature based image registration, Journal of Mathematical Imaging and Vision, 18 (2003), pp. 81-85.

[18] M. A. Fischler AND R. A. Elschlager, The representation and matching of pictorial structures, IEEE Transactions on Computers, 22 (1973), pp. 67-92.

[19] C. Frohn-Schauf, S. Henn, L. Hömke, and K. Witsch, Total variation based image registration, in Image Processing Based on Partial Differential Equations, Springer Berlin Heidelberg, 2007, pp. 343-361.

[20] C. Frohn-Schauf, S. Henn, and K. Witsch, Multigrid based total variation image registration, Computing and Visualization in Science, 11 (2008).

[21] R. Glowinski and P. Le Tallec, Augmented Lagrangian and Operator-Splitting Methods in Nonlinear Mechanics, Society for Industrial and Applied Mathematics, 1989.

[22] G. Y. Gu, B. S. He, AND X. M. YuAn, Customized proximal point algorithms for linearly constrained convex minimization and saddle-point problems: a unified approach, Comput. Optim. Appl., 59 (2014), pp. 135161.

[23] E. Haber AND J. Modersitzki, Image registration with guaranteed displacement regularity, 71, pp. 361-372.

[24] - Numerical methods for volume preserving image registration, 20, pp. 1621-1638.

[25] E. Haber ANd J. Modersitzki, Numerical methods for volume preserving image registration, Inverse Problems, 20 (2004), pp. 1621-1638.

[26] G. Hermosillo, C. Chefd'hotel, and O. Faugeras, Variational methods for multimodal image matching, International Journal of Computer Vision, 50 (2002), pp. 329-343.

[27] D. Hill, P. G. Batchelor, M. Holden, and D. J. Hawkes, Medical image registration, Physics in Medicine and Biology, 46 (2001).

[28] H. Y. Hsiao, C. Y. Hsieh, X. Chen, Y. Y. Gong, X. N. Luo, and G. J. Liao, New development of nonrigid registration, The Anziam Journal, 55 (2014), pp. 289-297.

[29] S. C. Joshi And M. I. Miller, Landmark matching via large deformation diffeomorphisms, IEEE Transactions on Image Processing, 9 (2000), pp. 1357-1370.

[30] B. Karacali and C. Davatzikos, Estimating topology preserving and smooth displacement fields, IEEE Transactions on Medical Imaging, 23 (2004), pp. 868-880. 
[31] K. C. LAm AND L. M. Lui, Landmark- and intensity-based registration with large deformations via quasiconformal maps, SIAM Journal on Imaging Sciences, 7 (2014), pp. 2364-2392.

[32] Y. T. LEe, K. C. LAm, AND L. M. LuI, Landmark-matching transformation with large deformation via $n$ dimensional quasi-conformal maps, Journal of Scientific Computing, 67 (2016), pp. 926-954.

[33] H. Li, J. Liu, L. Cui, H. HuAng, And X.-C. TAI, Volume preserving image segmentation with entropy regularized optimal transport and its applications in deep learning, 71, p. 102845.

[34] G. J. Liao, X. X. Cai, D. Fleitas, X. N. Luo, J. M. Wang, and J. X. Xue, Optimal control approach to data set alignment, Applied Mathematics Letters, 21 (2008), pp. 898-905.

[35] G. J. Liao and J. Z. Su, Grid generation via deformation, Applied Mathematics Letters, 5 (1992), pp. 27-29.

[36] J. Liu, New development of the deformation method, PhD thesis, University of Texas, 2006.

[37] M. Z. LiU, Total Bregman divergence, a robust divergence measure, and its applications, University of Florida, 2011.

[38] A. Mang AND L. Ruthotto, A lagrangian gauss-newton-krylov solver for mass- and intensity-preserving diffeomorphic image registration, SIAM Journal on Scientific Computing, 39 (2017).

[39] T. Mansi, X. Pennec, M. Sermesant, H. Delingette, and N. Ayache, ilogdemons: A demons-based registration algorithm for tracking incompressible elastic biological tissues, International Journal of Computer Vision, 92 (2011), pp. 92-111.

[40] J. Modersitzki, Numerical methods for image registration, Oxford University Press, 2004.

[41] - Fair: Flexible algorithms for image registration, (2008).

[42] C. S. Moore, G. P. Liney, And A. W. BeAvis, Quality assurance of registration of ct and mri data sets for treatment planning of radiotherapy for head and neck cancers, Journal of Applied Clinical Medical Physics, 5, pp. 25-35.

[43] A. Roche, G. Malandain, N. Ayache, and X. Pennec, Multimodal Image Registration by Maximization of the Correlation Ratio, Tech. Rep. RR-3378, INRIA, Aug. 1998.

[44] A. Salgovic, V. Hlavacek, and J. Ilavsky, Global simulation of counter current separation processes via one-parameter imbedding techniques, Chemical Engineering Science, 36 (1981), pp. 1599 - 1604.

[45] A. Sotiras, C. Davatzikos, and N. Paragios, Deformable medical image registration: A survey, IEEE Transactions on Medical Imaging, 32 (2013), pp. 1153-1190.

[46] J. ThIRION, Image matching as a diffusion process: an analogy with maxwell's demons, Medical Image Analysis, 2 (1998), pp. 243-260.

[47] A. Trouve, Diffeomorphisms groups and pattern matching in image analysis, International Journal of Computer Vision, 28 (1998), pp. 213-221.

[48] T. Vercauteren, X. Pennec, A. Perchant, and N. Ayache, Non-parametric diffeomorphic image registration with the demons algorithm, in International Conference on Medical Image Computing and ComputerAssisted Intervention, Springer, 2007, pp. 319-326.

[49] H. Wang, L. Dong, J. Daniel, R. Mohan, A. S. Garden, K. K. Ang, D. A. Kuban, M. Bonnen, J. Y. ChAng, AND R. Cheung, Validation of an accelerated demons algorithm for deformable image registration in radiation therapy, Physics in Medicine \& Biology, 50 (2005), p. 2887.

[50] C. Wu AND X.-C. TAI, Augmented Lagrangian Method, Dual Methods, and Split Bregman Iteration for ROF, Vectorial TV, and High Order Models, 3, pp. 300-339.

[51] I. Yanovsky, S. Osher, P. M. Thompson, and A. D. Leow, Log-unbiased large-deformation image registration, in Visapp: Second International Conference on Computer Vision Theory \& Applications, 2007.

[52] E. ZEIDLER, Nonlinear functional analysis and its applications III: Variational methods and optimization, Springer-Verlag, New York, 1985.

[53] D. P. Zhang and K. Chen, A novel diffeomorphic model for image registration and its algorithm, Journal of Mathematical Imaging and Vision, 60 (2018), pp. 1261-1283.

[54] J. Zhang, K. Chen, AND B. YU, An improved discontinuity-preserving image registration model and its fast algorithm, Applied Mathematical Modelling, 40 (2016), pp. 10740 - 10759.

[55] J. P. Zhang AND K. Chen, Variational image registration by a total fractional-order variation model, Journal of Computational Physics, 293 (2015), pp. $442-461$. 